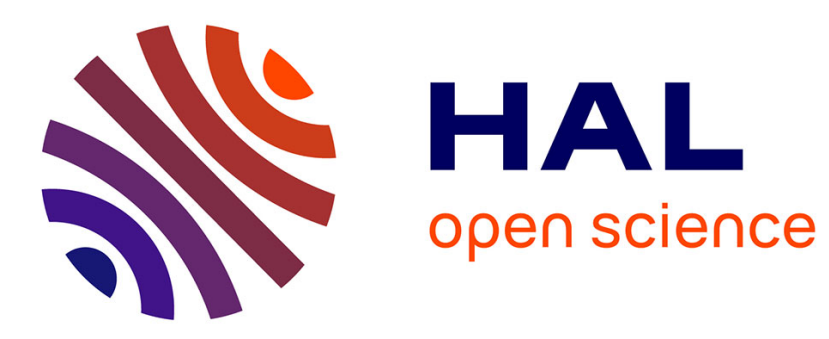

\title{
Homogenization of a Conductive, Convective and Radiative Heat Transfer Problem in a Heterogeneous Domain
}

Grégoire Allaire, Zakaria Habibi

\section{- To cite this version:}

Grégoire Allaire, Zakaria Habibi. Homogenization of a Conductive, Convective and Radiative Heat Transfer Problem in a Heterogeneous Domain. SIAM Journal on Mathematical Analysis, 2013, 45 (3), pp.1136-1178. hal-00932950

\section{HAL Id: hal-00932950 https://hal.science/hal-00932950}

Submitted on 18 Jan 2014

HAL is a multi-disciplinary open access archive for the deposit and dissemination of scientific research documents, whether they are published or not. The documents may come from teaching and research institutions in France or abroad, or from public or private research centers.
L'archive ouverte pluridisciplinaire HAL, est destinée au dépôt et à la diffusion de documents scientifiques de niveau recherche, publiés ou non, émanant des établissements d'enseignement et de recherche français ou étrangers, des laboratoires publics ou privés. 


\title{
Homogenization of a Conductive, Convective and Radiative Heat Transfer Problem in a Heterogeneous Domain*
}

\author{
Grégoire Allaire ${ }^{\dagger}$ and Zakaria Habibi ${ }^{\ddagger}$
}

January 21, 2013

\begin{abstract}
We are interested in the homogenization of heat transfer in periodic porous media where the fluid part is made of long thin parallel cylinders, the diameter of which is of the same order than the period. The heat is transported by conduction in the solid part of the domain and by conduction, convection and radiative transfer in the fluid part (the cylinders). A non-local boundary condition models the radiative heat transfer on the cylinder walls. To obtain the homogenized problem we first use a formal two-scale asymptotic expansion method. The resulting effective model is a convectiondiffusion equation posed in a homogeneous domain with homogenized coefficients evaluated by solving so-called cell problems where radiative transfer is taken into account. In a second step we rigorously justify the homogenization process by using the notion of two-scale convergence. One feature of this work is that it combines homogenization with a $3 \mathrm{D}$ to $2 \mathrm{D}$ asymptotic analysis since the radiative transfer in the limit cell problem is purely two-dimensional. Eventually, we provide some 3D numerical results in order to show the convergence and the computational advantages of our homogenization method.
\end{abstract}

Key words : Periodic homogenization, two-scale convergence, heat transfer.

\section{Introduction}

We study heat transfer in a very heterogeneous periodic porous medium. Since the ratio of the heterogeneities period with the characteristic length-scale of the domain, denoted by $\epsilon$, is very small in practice, a direct numerical simulation of this phenomenon is either out of reach or very time consuming on any computer (especially in 3D). Therefore, the original heterogeneous problem should be replaced by an homogeneous averaged (or effective, or homogenized) one. The goal of homogenization theory [8], [10], [15], [23], [25], [31], [32] is to provide a systematic way of finding such effective problems, of reconstructing an accurate solution by introducing so-called correctors and of rigorously justifying such an approximation by establishing convergence theorems and error estimates. The purpose of this paper is to carry on this program for a model of conductive, convective and radiative heat transfer in a 3D solid domain, periodically perforated by thin parallel cylinders in which a gas is flowing (see Figure 1 for a sketch of the geometry). Convection and radiative transfer are taking place only in the gas which is assumed to be transparent for radiation and with a very small bulk diffusivity. Therefore, the radiative transfer is modelled by a non-local boundary condition on the cylinder walls.

Although there are many possible physical motivations for this study, we focus on its application to the nuclear reactor industry and especially to the so-called gas-cooled reactors [19] which are a promising concept for the 4 th generation reactors. The periodic porous medium in our study is the

${ }^{*}$ This work has been supported by the French Atomic Energy and Alternative Energy Commission, DEN/DM2S at CEA Saclay.

${ }^{\dagger}$ CMAP, Ecole Polytechnique, 91128 Palaiseau, \& DM2S, CEA Saclay, 91191 Gif sur Yvette, gregoire.allaire@polytechnique.fr

${ }^{\ddagger}$ DM2S/SFME/LTMF, CEA Saclay, 91191 Gif sur Yvette, \& CMAP, Ecole Polytechnique, 91128 Palaiseau, zakaria.habibi@polytechnique.edu 
core of such a gas-cooled reactor. It is typically made of many prismatic blocks of graphite in which are inserted the nuclear fuel compact. Each block is periodically perforated by many small channels where the coolant (Helium) flows (the number of these gas cylinders is of the order of $10^{4}$ at least). Although the solid matrix of the porous medium is itself heterogeneous (a mixture of graphite and of nuclear materials), we simplify the exposition by assuming it is already homogenized and thus homogeneous. The analysis would not be much more complicated otherwise but certainly less clear for the reader. In other words, we assume that the only source of heterogeneities is coming from the geometry of the porous medium which is a fine mixture of solid and fluid parts. Since the total number of cylinders is very large and their diameter is very small compared to the size of the core, the numerical simulation of this problem requires a very fine mesh and thus a very expensive computational cost for a real geometry of a reactor core (all the more since the radiative transfer is modelled by an integral operator yielding dense discretization matrices). Therefore, our goal is to find a simpler homogenized model in an equivalent continuous domain and, specifically, to give a clear definition of the resulting effective parameters as well as a detailed reconstruction of an approximate solution (involving local correctors that take into account the geometry variation).

A similar study, in a simplified 2D setting, has previously appeared in [3]. In this reference, the 2D domain was a cross section of the reactor core (perpendicular to the cylinders) so that the fluid part was a periodic collection of isolated disks. Furthermore, convection and diffusion were neglected in the gas. Therefore, the main novelties of the present paper is, first, to take into account convection and diffusion in the fluid, second and most importantly, to consider a porous medium perforated by cylinders instead of disks. This last generalization is not at all a simple extension of the previous results of [3]. It turns out that [3] can easily be extended to a periodic distribution of spherical holes in 3D. On the contrary, in the case of cylinders, since periodicity takes place only in the transverse directions and the holes are not isolated, but rather connected, in the axial direction, a new phenomenon takes place which corresponds to a dimension reduction for the radiative operator from $3 \mathrm{D}$ to $2 \mathrm{D}$. In other words, our asymptotic analysis is not only a problem of homogenization but also of singular perturbation. The issue of dimension reduction is well-known in solid mechanics, where it is a basic ingredient to deduce plate or shell models from 3D elasticity when the thickness of the structure is going to zero (see e.g. [14]). Here, the reason for this dimension reduction is that, in the homogenization process, the cylinders become infinitely long compared to their diameter (which goes to zero): thus, at a microscopic scale the $3 \mathrm{D}$ radiative operator is asymptotically invariant along the axis of these cylinders and, in the limit, degenerates to a $2 \mathrm{D}$ radiative operator. Furthermore, some radiations are escaping from the cylinders by their extremities: asymptotically it yields an additional vertical homogenized diffusivity which was, of course, not seen in the $2 \mathrm{D}$ setting of [3]. Overall our homogenized model is new, quite surprising and not intuitive, even in light of [3].

There are a number of other previous contributions on the homogenization of radiative transfer which all correspond to different geometries or scalings of various parameters [6], [7], [9], [13]. Let us also mention that there is a huge literature on the homogenization in perforated domains or porous media (see [23] and references therein, [16], [18] for the case of non-linear Neumann boundary conditions).

The paper is organized as follows. In Section 2, we give a precise definition of the geometry and of the heat transfer model, see (8). In particular we discuss our scaling assumptions in terms of the small parameter $\epsilon$. Furthermore, various properties of the radiative operator are recalled. It is an integral operator, the kernel of which is called the view factor (it amounts to quantify how a point on the cylinder wall is illuminated by the other points on this surface). A key ingredient for the sequel is proved in Lemma 2.1: an asymptotic expansion of the 3D view factor, integrated along the cylinder axis, is established in terms of the 2D view factor. Section 3 is devoted to the formal method of two-scale asymptotic expansions applied to our problem. Its main result is Proposition 3.1 which gives the precise form of the homogenized problem. Furthermore, it also furnishes the so-called cell problems which define the corrector term for the homogenized solution. It is at the basis of a reconstruction process for an accurate and detailed approximate solution. We emphasize that the 
application of the formal method of two-scale asymptotic expansions is not standard for two reasons. First, to minimize the number of required terms in the resulting cascade of equations, we rely on a variant of the method, suggested by J.-L. Lions [26], which amounts to introduce an ansatz in the variational formulation rather than in the strong form of the equations. Second, we must combine this ansatz with the dimension reduction argument for the radiative operator as given by the technical Lemma 2.1. Section 4 provides a rigorous mathematical justification of the homogenization process by using the method of two-scale convergence [1], [30]. Our main result is Theorem 4.2 which confirms the statement of Proposition 3.1. A formal generalization to the non-linear case is briefly sketched in Section 5. Indeed, our mathematical rigorous justification holds true only for a linear model so we choose to expose this setting. However, the true physical model of radiative transfer is non-linear since the emitted radiations are following Stefan-Boltzmann law of proportionality to the 4th power of temperature. Taking into account this non-linearity is not difficult for the formal method of two-scale asymptotic expansions. Thus we give the homogenized and cell problems in this case too, all the more since all our numerical computations are performed in this non-linear setting. In Section 6, we present some numerical results for data corresponding to gas-cooled reactors. In particular we show that the error between the exact and reconstructed solutions, as a function of the small parameter $\epsilon$, is as expected of order 1 or $1 / 2$, depending on the choice of norm.

\section{Setting of the problem}

The goal of this section is to define the geometry of the periodic porous medium and to introduce the model of conductive, convective and radiative heat transfer.

\subsection{Geometry}

For simplicity we consider a rectangular open set $\Omega=\prod_{j=1}^{3}\left(0, L_{j}\right)$ where $L_{j}>0$ are positive lengths. It is however essential that the domain $\Omega$ be a cylinder with axis in the third direction, namely that its geometry is invariant by translation along $x_{3}$. The rectangular basis $\prod_{j=1}^{2}\left(0, L_{j}\right)$ is periodically divided in $N(\epsilon)$ small cells $\left(\Lambda_{\epsilon, i}\right)_{i=1 \ldots N(\epsilon)}$, each of them being equal, up to a translation and rescaling by a factor $\epsilon$, to the same unit periodicity cell $\Lambda=\prod_{j=1}^{2}\left(0, l_{j}\right)$ with $l_{j}>0$. By construction, the domain $\Omega$ is periodic in the two first directions and is invariant by translation in the third one. To avoid unnecessary complications with boundary layers (and because this is the case in the physical problem which motivates this study) we assume that the sequence of small positive parameters $\epsilon$, going to zero, is such that the basis of $\Omega$ is made up of entire cells only, namely $L_{j} /\left(\epsilon l_{j}\right)$ is an integer for any $j=1,2$. The cell $\Lambda$ is decomposed in two parts: the holes $\Lambda^{F}$ occupied by a fluid (see Figures 1 and 2) and the solid matrix $\Lambda^{S}$. We denote by $\gamma$ the boundary between $\Lambda^{S}$ and $\Lambda^{F}$. Then, we define the fluid domain $\Omega_{\epsilon}^{F}$ as the cylindrical domain with basis composed by the collection of $\Lambda_{\epsilon, i}^{F}$ and the solid domain $\Omega_{\epsilon}^{S}$ as the cylindrical domain with basis composed by the collection of $\Lambda_{\epsilon, i}^{S}$, where $\Lambda_{\epsilon, i}^{F, S}$ are the translated and rescaled version of $\Lambda^{F, S}$ for $i=1 \ldots N(\epsilon)$ (similar to the correspondence between $\Lambda_{\epsilon, i}$ and $\Lambda$ ). In summary we have

$\Omega_{\epsilon}^{F}=\bigcup_{i=1}^{N(\epsilon)} \Lambda_{\epsilon, i}^{F} \times\left(0, L_{3}\right), \quad \Omega_{\epsilon}^{S}=\Omega \backslash \Omega_{\epsilon}^{F}=\bigcup_{i=1}^{N(\epsilon)} \Lambda_{\epsilon, i}^{S} \times\left(0, L_{3}\right), \quad \gamma_{\epsilon}=\bigcup_{i=1}^{N(\epsilon)} \gamma_{\epsilon, i}, \quad \Gamma_{\epsilon}=\gamma_{\epsilon} \times\left(0, L_{3}\right)$.

For each plane cell $\Lambda_{\epsilon, i}$, the center of mass $x_{0, i}^{\prime}$ of the boundary $\gamma_{\epsilon, i}$ is defined by

$$
\int_{\gamma_{\epsilon, i}}\left(s^{\prime}-x_{0, i}^{\prime}\right) d s^{\prime}=0
$$

For any point $x=\left(x_{1}, x_{2}, x_{3}\right) \in \mathbb{R}^{3}$, we denote by $x^{\prime}$ its two first components in $\mathbb{R}^{2}$ such that $x=\left(x^{\prime}, x_{3}\right)$. We introduce the linear projection operator $P$ from $\mathbb{R}^{3}$ to $\mathbb{R}^{2}$ and its adjoint, the 
extension operator $E$ from $\mathbb{R}^{2}$ to $\mathbb{R}^{3}$, defined by

$$
P\left(\begin{array}{l}
v_{1} \\
v_{2} \\
v_{3}
\end{array}\right)=\left(\begin{array}{l}
v_{1} \\
v_{2}
\end{array}\right) \quad \text { and } \quad E\left(\begin{array}{l}
v_{1} \\
v_{2}
\end{array}\right)=\left(\begin{array}{c}
v_{1} \\
v_{2} \\
0
\end{array}\right) .
$$

Eventually, we denote by $\nabla_{x^{\prime}}$ the $2 \mathrm{D}$ gradient operator which we shall often identify to its extension $E \nabla_{x^{\prime}}$. Similarly, for a 3D vector field $F\left(x^{\prime}, x_{3}\right)$ we shall use the notation $\operatorname{div}_{x^{\prime}} F$ for $\operatorname{div}_{x}(P F)$.

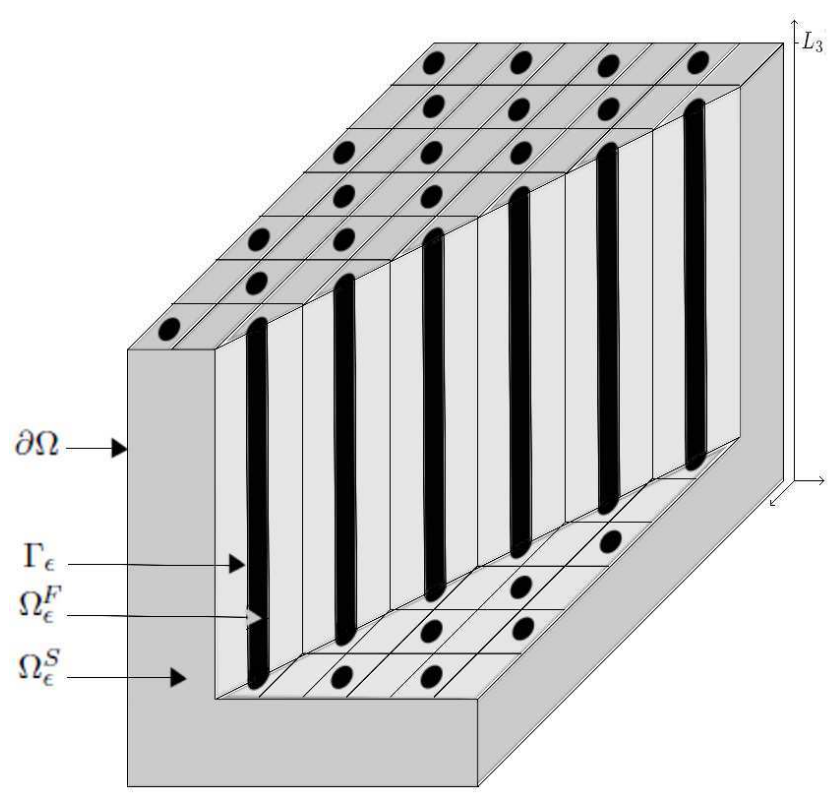

Figure 1: Periodic domain for a gas cooled reactor core

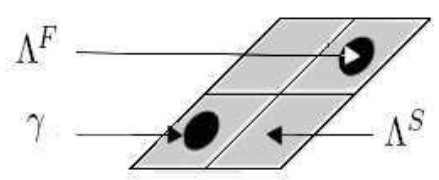

Figure 2: 2D reference cell for a gas cooled reactor core

\subsection{Governing equations}

There is a vast literature on heat transfer and we refer the interested reader to [12], [27], [33] for an introduction to the modelling of radiative transfer. We denote by $T_{\epsilon}$ the temperature in the domain $\Omega$ which can be decomposed as

$$
T_{\epsilon}=\left\{\begin{array}{lll}
T_{\epsilon}^{S} & \text { in } & \Omega_{\epsilon}^{S} \\
T_{\epsilon}^{F} & \text { in } & \Omega_{\epsilon}^{F}
\end{array}\right.
$$

where $T_{\epsilon}$ is continuous through the interface $\Gamma_{\epsilon}$.

Convection takes place only in the thin vertical cylinders $\Omega_{\epsilon}^{F}$ occupied by the fluid. We thus introduce a given fluid velocity

$$
V_{\epsilon}(x)=V\left(x, \frac{x^{\prime}}{\epsilon}\right) \quad \text { in } \quad \Omega_{\epsilon}^{F},
$$

where the continuous vector field $V\left(x, y^{\prime}\right)$, defined in $\Omega \times \Lambda^{F}$, is periodic with respect to $y^{\prime}$ and satisfies the two incompressibility constraints

$$
\operatorname{div}_{x} V=0 \text { and } \operatorname{div}_{y^{\prime}} V=0 \text { in } \Omega \times \Lambda^{F}, \text { and } V \cdot n=0 \text { on } \Omega \times \gamma
$$


where $n$ is the unit outward normal (from $\Lambda^{S}$ to $\Lambda^{F}$ ) on $\gamma$. A typical example of such a velocity field is $V=\left(V^{\prime}\left(x_{3}, y^{\prime}\right), V_{3}\left(x^{\prime}, y^{\prime}\right)\right)$ with $V^{\prime}=\left(V_{1}, V_{2}\right), \operatorname{div}_{y^{\prime}} V^{\prime}=0$ and $V^{\prime} \cdot n=0$ on $\gamma$.

The thermal diffusion is assumed to be much smaller in the fluid than in the solid. More precisely we assume that it is of order 1 in $\Omega_{\epsilon}^{S}$ and of order $\epsilon$ in $\Omega_{\epsilon}^{F}$. The conductivity tensor is thus defined by

$$
K_{\epsilon}(x)=\left\{\begin{array}{l}
K_{\epsilon}^{S}(x)=K^{S}\left(x, \frac{x^{\prime}}{\epsilon}\right) \quad \text { in } \Omega_{\epsilon}^{S}, \\
\epsilon K_{\epsilon}^{F}(x)=\epsilon K^{F}\left(x, \frac{x^{\prime}}{\epsilon}\right) \quad \text { in } \Omega_{\epsilon}^{F},
\end{array}\right.
$$

where $K^{S}\left(x, y^{\prime}\right), K^{F}\left(x, y^{\prime}\right)$ are periodic symmetric positive definite tensors defined in the unit cell $Y$, satisfying

$$
\forall v \in \mathbb{R}^{3}, \forall y^{\prime} \in \Lambda, \forall x \in \Omega, \quad \alpha|v|^{2} \leq \sum_{i, j=1}^{3} K_{i, j}^{F, S}\left(x, y^{\prime}\right) v_{i} v_{j} \leq \beta|v|^{2},
$$

for some constants $0<\alpha \leq \beta$. The choice of the $\epsilon$ scaling in (3) is made in order to have a dominant convection in the fluid part at the macroscopic scale. However, at the microscopic scale the convection and the diffusion are balanced as will be clear later.

The fluid is assumed to be almost transparent, so that heat can also be transported by radiative transfer in $\Omega_{\epsilon}^{F}$. This radiative effect is modelled by a non local boundary condition on the interface $\Gamma_{\epsilon}$ between $\Omega_{\epsilon}^{F}$ and $\Omega_{\epsilon}^{S}$. More precisely, in addition to the continuity of temperature we write a balance of heat fluxes on the interface

$$
T_{\epsilon}^{S}=T_{\epsilon}^{F} \quad \text { and } \quad-K_{\epsilon}^{S} \nabla T_{\epsilon}^{S} \cdot n=-\epsilon K_{\epsilon}^{F} \nabla T_{\epsilon}^{F} \cdot n+\frac{\sigma}{\epsilon} G_{\epsilon}\left(T_{\epsilon}^{F}\right) \quad \text { on } \Gamma_{\epsilon},
$$

where $\sigma>0$ is a given positive constant and $G_{\epsilon}$ is the radiative operator defined by

$$
G_{\epsilon}\left(T_{\epsilon}\right)(s)=T_{\epsilon}(s)-\int_{\Gamma_{\epsilon, i}} T_{\epsilon}(x) F(s, x) d x=\left(I d-\zeta_{\epsilon}\right) T_{\epsilon}(s) \quad \forall s \in \Gamma_{\epsilon, i},
$$

with

$$
\zeta_{\epsilon}(f)(s)=\int_{\Gamma_{\epsilon, i}} F(s, x) f(x) d x .
$$

The scaling $\epsilon^{-1}$ in front of the radiative operator $G_{\epsilon}$ in (4) is chosen because it yields a perfect balance, in the limit as $\epsilon$ goes to zero, between the bulk heat conduction and the surface radiative transfer (this scaling was first proposed in [3] and is due to the fact that the operator $\left(I d-\zeta_{\epsilon}\right)$ has a non-trivial kernel, see Lemma 2.1). In (6) $F$ is the so-called view factor (see [27], [24], [22]). The view factor $F(s, x)$ is a geometrical quantity between two different points $s$ and $x$ of the same cylinder $\Gamma_{\epsilon, i}$. Its explicit formula for surfaces enclosing convex domains is in $3 \mathrm{D}$

$$
F(s, x):=F^{3 D}(s, x)=\frac{n_{x} \cdot(s-x) n_{s} \cdot(x-s)}{\pi|x-s|^{4}},
$$

where $n_{z}$ denotes the unit normal at the point $z$. In $2 \mathrm{D}$ the view factor is

$$
F(s, x):=F^{2 D}\left(s^{\prime}, x^{\prime}\right)=\frac{n_{x}^{\prime} \cdot\left(s^{\prime}-x^{\prime}\right) n_{s}^{\prime} \cdot\left(x^{\prime}-s^{\prime}\right)}{2\left|x^{\prime}-s^{\prime}\right|^{3}}
$$

and the operator in (6) is denoted by $\zeta_{\epsilon}^{2 D}$. Some useful properties of the view factor are given below in Lemma 2.1.

For simplicity we assume that the only heat source is a bulk density of thermal sources in the solid part, $f \in L^{2}(\Omega), f \geq 0$ and the external boundary condition is a simple Dirichlet condition. Eventually, the governing equations of our model are

$$
\left\{\begin{aligned}
-\operatorname{div}\left(K_{\epsilon}^{S} \nabla T_{\epsilon}^{S}\right) & =f & & \text { in } \Omega_{\epsilon}^{S} \\
-\operatorname{div}\left(\epsilon K_{\epsilon}^{F} \nabla T_{\epsilon}^{F}\right)+V_{\epsilon} \cdot \nabla T_{\epsilon}^{F} & =0 & & \text { in } \Omega_{\epsilon}^{F} \\
-K_{\epsilon}^{S} \nabla T_{\epsilon}^{S} \cdot n & =-\epsilon K_{\epsilon}^{F} \nabla T_{\epsilon}^{F} \cdot n+\frac{\sigma}{\epsilon} G_{\epsilon}\left(T_{\epsilon}^{F}\right) & & \text { on } \Gamma_{\epsilon} \\
T_{\epsilon}^{S} & =T_{\epsilon}^{F} & & \text { on } \Gamma_{\epsilon} \\
T_{\epsilon} & =0 & & \text { on } \partial \Omega .
\end{aligned}\right.
$$


Proposition 2.1. The boundary value problem (8) admits a unique solution $T_{\epsilon}$ in $H_{0}^{1}(\Omega)$.

Proof This is a classical result (see [3] if necessary) by application of the Lax-Milgram lemma. The main point is that the operator $G_{\epsilon}$ is self-adjoint and non-negative, as stated in Lemma 2.1 below.

Remark 2.1. The solution of (8) satisfies the maximum principle, namely $f \geq 0$ in $\Omega$ implies that $T_{\epsilon} \geq 0$ in $\Omega$ (see [33]). However, we shall not use this property in the sequel.

Remark 2.2. The radiation operator introduced in (5) is a linear operator: this is clearly a simplifying assumption. Actually, the true physical radiation operator is non-linear and defined, on each $\Gamma_{\epsilon, i}, 1 \leq$ $i \leq N(\epsilon)$, by

$$
G_{\epsilon}\left(T_{\epsilon}\right)=e\left(I d-\zeta_{\epsilon}\right)\left(I d-(1-e) \zeta_{\epsilon}\right)^{-1}\left(T_{\epsilon}^{4}\right) .
$$

where $\zeta_{\epsilon}$ is the operator defined by (6). To simplify the exposition, we focus on the case of so-called black walls, i.e., we assume that the emissivity is $e=1$ (we can find in [7] a study of this kind of problems when the emissivity depends on the radiation frequency). However, our analysis can be extended straightforwardly to the other cases $0<e<1$ (see e.g. [20]). The formal two-scale asymptotic expansion method can also be extended to the above non-linear operator, at the price of more tedious computations [20]. However, the rigorous justification of the homogenization process is, for the moment, available only for the linearized form of the radiation operator. Therefore we content ourselves in exposing the homogenization process for the linear case. Nevertheless, in Section 5 we indicate how our results can be generalized to the above non-linear setting. Furthermore, our numerical results in Section 6 are obtained in the non-linear case which is more realistic from a physical point of view.

\subsection{Properties of the view factor}

We recall and establish some useful properties of the view factor that we will use later.

Lemma 2.1. For points $x$ and $s$ belonging to the same cylinder $\Gamma_{\epsilon, i}$, the view factor $F(s, x)$ satisfies 1.

$$
F(s, x) \geq 0, \quad F(s, x)=F(x, s)
$$

2.

$$
\int_{\gamma_{\epsilon, i}} F^{2 D}\left(s^{\prime}, x^{\prime}\right) d s^{\prime}=1
$$

3. as an operator from $L^{2}$ into itself,

$$
\left\|\zeta_{\epsilon}\right\| \leq 1
$$

4.

$$
\int_{\gamma_{\epsilon, i}} \int_{\gamma_{\epsilon, i}}\left(x^{\prime}-x_{0, i}^{\prime}\right) F^{2 D}\left(s^{\prime}, x^{\prime}\right) d x^{\prime} d s^{\prime}=0
$$

5.

$$
\operatorname{ker}\left(I d-\zeta_{\epsilon}^{2 D}\right)=\mathbb{R}
$$

6. the radiative operator $G_{\epsilon}$ is self-adjoint on $L^{2}\left(\Gamma_{\epsilon, i}\right)$ and non-negative in the sense that

$$
\int_{\Gamma_{\epsilon, i}} G_{\epsilon}(f) f d s \geq 0 \quad \forall f \in L^{2}\left(\Gamma_{\epsilon, i}\right)
$$


7. for any given $s_{3} \in(0, L)$,

$$
\int_{0}^{L} F^{3 D}(s, x) d x_{3}=F^{2 D}\left(s^{\prime}, x^{\prime}\right)+\mathcal{O}\left(\frac{\epsilon^{2}}{L^{3}}\right),
$$

8. for any function $g \in C^{3}(0, L)$ with compact support in $(0, L)$,

$$
\int_{0}^{L} g\left(x_{3}\right) F^{3 D}(s, x) d x_{3}=F^{2 D}\left(s^{\prime}, x^{\prime}\right)\left(g\left(s_{3}\right)+\frac{\left|x^{\prime}-s^{\prime}\right|^{2}}{2} g^{\prime \prime}\left(s_{3}\right)+\mathcal{O}\left(\epsilon^{p}\right)\right),
$$

where any $0<p<3$ is admissible and $g^{\prime \prime}$ denotes the second derivative of $g$. Furthermore, for any function $f \in L^{\infty}(0, L)$, we have

$$
\begin{aligned}
\int_{0}^{L} \int_{0}^{L} f\left(x_{3}\right) g\left(s_{3}\right) F^{3 D}(s, x) d x_{3} d s_{3} & =F^{2 D}\left(s^{\prime}, x^{\prime}\right)\left(\int_{0}^{L} f\left(x_{3}\right) g\left(x_{3}\right) d x_{3}\right. \\
& \left.+\frac{1}{2}\left|x^{\prime}-s^{\prime}\right|^{2} \int_{0}^{L} f\left(x_{3}\right) g^{\prime \prime}\left(x_{3}\right) d x_{3}+\mathcal{O}\left(\epsilon^{p}\right)\right) .
\end{aligned}
$$

Remark 2.3. The surface $\Gamma_{\epsilon, i}$ of each cylinder is not closed (it is only the lateral boundary and the two end cross-sections are missing). Therefore, the second property of Lemma 2.1 does not hold in $3 D$, namely

$$
\int_{\Gamma_{\epsilon, i}} F^{3 D}\left(s^{\prime}, x^{\prime}\right) d s^{\prime} \neq 1
$$

Remark 2.4. The asymptotic properties (14) can be physically interpreted by saying that in a thin and long cylinder the $3 D$ view factor are well approximated by the $2 D$ view factor, upon vertical integration.

Since the surface $\Gamma_{\epsilon, i}$ is open at its extremities, there is some leakage of the radiated energy. The asymptotic property (15) and (16) take into account the quantification of this leakage which corresponds to a diffusive corrector term in the $x_{3}$ direction (remember that $\left|x^{\prime}-s^{\prime}\right|^{2}$ is of the order of $\epsilon^{2}$ ).

Proof The six first properties are classical and may be found in [20]. The proof of (14) follows from a change of variables and a Taylor expansion. At this point, the assumption that $s_{3}$ does not depend on $\epsilon$ and is different from the two end points 0 and $L$ is crucial. Indeed, because the cylinder $\Gamma_{\epsilon, i}$ is vertical, we have $n_{s_{3}}=n_{x_{3}}=0$ and

$$
I=\int_{0}^{L} \frac{n_{x} \cdot(s-x) n_{s} \cdot(x-s)}{\pi|x-s|^{4}} d x_{3}=\frac{n_{x}^{\prime} \cdot\left(s^{\prime}-x^{\prime}\right) n_{s}^{\prime} \cdot\left(x^{\prime}-s^{\prime}\right)}{\pi\left(x^{\prime}-s^{\prime}\right)^{4}} \int_{0}^{L} \frac{1}{\left(1+\frac{\left(x_{3}-s_{3}\right)^{2}}{\left|x^{\prime}-s^{\prime}\right|^{2}}\right)^{2}} d x_{3} .
$$

By the change of variables

$$
z=\frac{x_{3}-s_{3}}{\alpha}, \quad \text { where } \quad \alpha=\left|x^{\prime}-s^{\prime}\right|
$$

and integration, we obtain

$$
I=\frac{2}{\pi} F^{2 D}\left(s^{\prime}, x^{\prime}\right)\left(h_{1}\left(\frac{L-s_{3}}{\left|x^{\prime}-s^{\prime}\right|}\right)-h_{1}\left(\frac{-s_{3}}{\left|x^{\prime}-s^{\prime}\right|}\right)\right)
$$

where $h_{1}(z)$ is the primitive of the previous integrand given by

$$
h_{1}(z)=\frac{1}{2}\left(\frac{z}{z^{2}+1}+\arctan (z)\right) .
$$


By Taylor expansion we get

$$
h_{1}(z)=\left\{\begin{array}{rr}
+\frac{\pi}{2}+\mathcal{O}\left(z^{-3}\right) & \text { when } z \rightarrow+\infty \\
-\frac{\pi}{2}+\mathcal{O}\left(z^{-3}\right) & \text { when } z \rightarrow-\infty
\end{array}\right.
$$

Since $\left|x^{\prime}-s^{\prime}\right|=\mathcal{O}(\epsilon), s_{3}=\mathcal{O}(L)$ and $F^{2 D}\left(s^{\prime}, x^{\prime}\right)=\mathcal{O}\left(\epsilon^{-1}\right)$, we deduce (14).

The proof of (15) is a little more difficult although the strategy is the same. Let us notice that the assumption of compact support for $g$ allows us to avoid difficulties coming from the case when $s_{3}=0$ or $s_{3}=L$. By the same change of variables (17) we obtain

$$
I=\int_{0}^{L} g\left(x_{3}\right) F^{3 D}(s, x) d x_{3}=\frac{2}{\pi} F^{2 D}\left(x^{\prime}, s^{\prime}\right) \int_{\Delta} \frac{g\left(s_{3}+\alpha z\right)}{\left(1+z^{2}\right)^{2}} d z=\frac{2}{\pi} F^{2 D}\left(x^{\prime}, s^{\prime}\right) \widehat{I}
$$

where the domain of integration $\Delta$ is given by $\Delta=\left[\frac{-s_{3}}{\alpha}, \frac{L-s_{3}}{\alpha}\right]$. Remark that $\alpha=\mathcal{O}(\epsilon)$. By using a Taylor expansion in a neighbourhood of $s_{3}$, we have

$$
g\left(s_{3}+\alpha z\right)=g\left(s_{3}\right)+\alpha z g^{\prime}\left(s_{3}\right)+\frac{1}{2} \alpha^{2} z^{2} g^{\prime \prime}\left(s_{3}\right)+\mathcal{O}\left(\alpha^{3} z^{3}\right),
$$

and $\widehat{I}$ becomes

$$
\widehat{I}=I_{1}+I_{2}+I_{3}+I_{4}
$$

where, $h_{1}(z)$ being given by (18),

$$
I_{1}=g\left(s_{3}\right) \int_{\Delta} \frac{1}{\left(1+z^{2}\right)^{2}} d z=\frac{g\left(s_{3}\right)}{2}\left(h_{1}\left(\frac{L-s_{3}}{\alpha}\right)-h_{1}\left(\frac{-s_{3}}{\alpha}\right)\right)=\frac{g\left(s_{3}\right)}{2}\left(\pi+\mathcal{O}\left(\alpha^{3}\right)\right) .
$$

On the other hand we get

$$
\begin{gathered}
I_{2}=\alpha g^{\prime}\left(s_{3}\right) \int_{\Delta} \frac{z}{\left(1+z^{2}\right)^{2}} d z=\alpha g^{\prime}\left(s_{3}\right)\left(h_{2}\left(\frac{L-s_{3}}{\alpha}\right)-h_{2}\left(\frac{-s_{3}}{\alpha}\right)\right)=\frac{\alpha g^{\prime}\left(s_{3}\right)}{2} \mathcal{O}\left(\alpha^{2}\right), \\
I_{3}=\frac{\alpha^{2}}{2} g^{\prime \prime}\left(s_{3}\right) \int_{D} \frac{z^{2}}{\left(1+z^{2}\right)^{2}} d z=\frac{\alpha^{2}}{2} g^{\prime \prime}\left(s_{3}\right)\left(h_{3}\left(\frac{L-s_{3}}{\alpha}\right)-h_{3}\left(\frac{-s_{3}}{\alpha}\right)\right)=\frac{\alpha^{2}}{4} g^{\prime \prime}\left(s_{3}\right)(\pi+\mathcal{O}(\alpha)),
\end{gathered}
$$

where we performed a Taylor expansion of $h_{2}(z)$ and $h_{3}(z)$ which are the primitives of the previous integrands in $I_{2}$ and $I_{3}$, respectively, given by

$$
h_{2}(z)=\frac{1}{2}\left(\frac{-1}{z^{2}+1}\right), \quad h_{3}(z)=\frac{1}{2}\left(\frac{-z}{z^{2}+1}+\arctan (z)\right) .
$$

The last integral in $(20)$ is of order $\mathcal{O}\left(\epsilon^{p}\right)$ for any $0<p<3$ because

$$
\left|I_{4}\right| \leq C \alpha^{3} \int_{\Delta} \frac{z^{3}}{\left(1+z^{2}\right)^{2}} d z \leq C \alpha^{3}\left(h_{4}\left(\frac{L-s_{3}}{\alpha}\right)-h_{4}\left(\frac{-s_{3}}{\alpha}\right)\right),
$$

where $h_{4}(z)$ is the primitive of the previous integrand given by

$$
h_{4}(z)=\frac{1}{2}\left(\log \left(z^{2}+1\right)+\frac{1}{1+z^{2}}\right)
$$

By a Taylor expansion of (21) when $z \rightarrow \pm \infty$ we get

$$
\left|I_{4}\right| \leq C \alpha^{3}|\log \alpha| \leq C \alpha^{p} \quad \forall 0<p<3 .
$$

Hence the result (15) since $\alpha=\mathcal{O}(\epsilon)$. Eventually, (16) is immediate using (15). 
Remark 2.5. If the function $f$ is smooth, by integration by parts (16) becomes

$$
\begin{aligned}
\int_{0}^{L} \int_{0}^{L} f\left(x_{3}\right) g\left(s_{3}\right) F^{3 D}(s, x) d x_{3} d s_{3} & =F^{2 D}\left(s^{\prime}, x^{\prime}\right)\left(\int_{0}^{L} f\left(x_{3}\right) g\left(x_{3}\right) d x_{3}\right. \\
& \left.-\frac{1}{2}\left|x^{\prime}-s^{\prime}\right|^{2} \int_{0}^{L} f^{\prime}\left(x_{3}\right) g^{\prime}\left(x_{3}\right) d x_{3}+\mathcal{O}\left(\epsilon^{p}\right)\right) .
\end{aligned}
$$

Actually, (22) can be proved directly with different smoothness assumptions: it holds true for $f$ and $g$ of class $C^{2}$, one of them being with compact support.

\section{Two-scale asymptotic expansion}

The homogenized problem can be obtained heuristically by the method of two-scale asymptotic expansion [10], [15], [31]. This method is based on the periodic assumption on the geometry of the porous medium. However here, because the radiative operator is only $2 \mathrm{D}$ periodic, we shall introduce a microscopic variable $y^{\prime}$ which is merely a $2 \mathrm{D}$ variable (in the plane perpendicular to the cylinders). Of course, denoting the space variable $x=\left(x^{\prime}, x_{3}\right)$, the fast and slow variables are related by $y^{\prime}=x^{\prime} / \epsilon$. The radiative operator is creating an additional difficulty: since the fluid part is made of thin and long cylinders, the $3 \mathrm{D}$ view factors will asymptotically be replaced by the $2 \mathrm{D}$ view factors (see Lemma 2.1). Therefore, our problem is not only an homogenization problem but it is also a singularly perturbed one. It can be compared to the dimension reduction issue in solid mechanics, i.e., how a plate or shell model can be deduced from a 3D elasticity one (see e.g. [14]).

The starting point of the method of two-scale asymptotic expansion is to assume that the solution $T_{\epsilon}$ of problem (8) is given by the series

$$
T_{\epsilon}=T_{0}(x)+\epsilon T_{1}\left(x, \frac{x^{\prime}}{\epsilon}\right)+\epsilon^{2} T_{2}\left(x, \frac{x^{\prime}}{\epsilon}\right)+\mathcal{O}\left(\epsilon^{3}\right)
$$

where, for $i=1,2, y^{\prime} \rightarrow T_{i}\left(x, y^{\prime}\right)$ is $\Lambda$-periodic and

$$
T_{i}\left(x, y^{\prime}\right)= \begin{cases}T_{i}^{S}\left(x, y^{\prime}\right) & \text { in } \Omega \times \Lambda^{S} \\ T_{i}^{F}\left(x, y^{\prime}\right) & \text { in } \Omega \times \Lambda^{F}\end{cases}
$$

with the continuity condition at the interface, $T_{i}^{S}\left(x, y^{\prime}\right)=T_{i}^{F}\left(x, y^{\prime}\right)$ on $\gamma=\partial \Lambda^{S} \cap \partial \Lambda^{F}$. As in the classical examples of homogenization, we assume that the first term of the asymptotic expansion $T_{0}$ depends only on the macroscopic variable $x$. As usual this property can be established by the same development as below if we had assumed rather that $T_{0} \equiv T_{0}\left(x, y^{\prime}\right)$.

Introducing (23) in the equations (8) of the model, we deduce the main result of this section.

Proposition 3.1. Under assumption (23), the zero-order term $T_{0}$ of the expansion for the solution $T_{\epsilon}$ of (8) is the solution of the homogenized problem

$$
\left\{\begin{aligned}
-\operatorname{div}\left(K^{*}(x) \nabla T_{0}(x)\right)+V^{*}(x) \cdot \nabla T_{0}(x) & =\theta f(x) & & \text { in } \Omega \\
T_{0}(x) & =0 & & \text { on } \partial \Omega
\end{aligned}\right.
$$

with the porosity factor $\theta=\left|\Lambda^{S}\right| /|\Lambda|$, the homogenized conductivity tensor $K^{*}$ given by its entries, for $j, k=1,2,3$,

$$
\begin{aligned}
K_{j, k}^{*}(x)=\frac{1}{|\Lambda|} & {\left[\int_{\Lambda^{S}} K^{S}\left(x, y^{\prime}\right)\left(e_{j}+\nabla_{y^{\prime}} \omega_{j}^{S}\left(x, y^{\prime}\right)\right) \cdot\left(e_{k}+\nabla_{y^{\prime}} \omega_{k}^{S}\left(x, y^{\prime}\right)\right) d y^{\prime}\right.} \\
& +\sigma \int_{\gamma}\left(I d-\zeta^{2 D}\right)\left(\omega_{k}^{S}\left(x, y^{\prime}\right)+y_{k}\right)\left(\omega_{j}^{S}\left(x, y^{\prime}\right)+y_{j}\right) d y^{\prime} \\
& \left.+\frac{\sigma}{2} \int_{\gamma} \int_{\gamma} F^{2 D}\left(s^{\prime}, y^{\prime}\right)\left|s^{\prime}-y^{\prime}\right|^{2} d y^{\prime} d s^{\prime} \delta_{j 3} \delta_{k 3}\right]
\end{aligned}
$$


and a vertical homogenized velocity given by

$$
V^{*}(x)=\frac{e_{3}}{|\Lambda|} \int_{\Lambda^{F}} V\left(x, y^{\prime}\right) \cdot e_{3} d y^{\prime}
$$

where $\zeta^{2 D}$ is the unit cell view factor operator defined by

$$
\zeta^{2 D}(\omega)\left(s^{\prime}\right)=\int_{\gamma} F^{2 D}\left(s^{\prime}, y^{\prime}\right) \omega\left(y^{\prime}\right) d y^{\prime}
$$

and, for $j=1,2,3, \omega_{j}\left(x, y^{\prime}\right)$ (equal to $\omega_{j}^{S}$ in $\Lambda^{S}$ and to $\omega_{j}^{F}$ in $\Lambda^{F}$ ) is the solution of the $2 D$ cell problem

$$
\left\{\begin{array}{rrr}
-\operatorname{div}_{y^{\prime}} P\left[K^{S}\left(x, y^{\prime}\right)\left(e_{j}+\nabla_{y^{\prime}} \omega_{j}^{S}\left(x, y^{\prime}\right)\right)\right] & =0 & \text { in } \Lambda^{S} \\
-\operatorname{div}_{y^{\prime}} P\left[K^{F}\left(x, y^{\prime}\right)\left(e_{j}+\nabla_{y^{\prime}} \omega_{j}^{F}\left(x, y^{\prime}\right)\right)\right]+V\left(x, y^{\prime}\right) \cdot\left(e_{j}+\nabla_{y^{\prime}} \omega_{j}^{F}\left(x, y^{\prime}\right)\right) & =0 & \text { in } \Lambda^{F} \\
-P\left[K^{S}\left(x, y^{\prime}\right)\left(e_{j}+\nabla_{y^{\prime}} \omega_{j}^{S}\left(x, y^{\prime}\right)\right)\right] \cdot n=\sigma\left(I d-\zeta^{2 D}\right) & \left(\omega_{j}^{S}\left(x, y^{\prime}\right)+y_{j}\right) & \text { on } \gamma \\
\omega_{j}^{F}\left(x, y^{\prime}\right) & =\omega_{j}^{S}\left(x, y^{\prime}\right) & \text { on } \gamma \\
y^{\prime} \mapsto \omega_{j}\left(x, y^{\prime}\right) & \text { is } \Lambda \text {-periodic, } &
\end{array}\right.
$$

where $P$ is the $3 D$ to $2 D$ projection operator defined by (2). Furthermore, $T_{1}$ is given by

$$
T_{1}\left(x, y^{\prime}\right)=\sum_{j=1}^{3} \omega_{j}\left(x, y^{\prime}\right) \frac{\partial T_{0}}{\partial x_{j}}(x) .
$$

Remark 3.1. As usual in homogenization, the cell problem (27) is a partial differential equation with respect to $y^{\prime}$ where $x$ plays the role of a parameter. It is proved to be well-posed in Lemma 3.1 below.

We emphasize that the cell problem in $\Lambda$ can be decoupled as two successive sub-problems in $\Lambda^{S}$ and $\Lambda^{F}$ respectively. First, we solve a cell problem in $\Lambda^{S}$ using the non local boundary condition on $\gamma$, independently of what happens in $\Lambda^{F}$. Second, we solve a cell problem in $\Lambda^{F}$ with the continuity boundary condition on $\gamma$ yielding a Dirichlet boundary condition. In particular, the homogenized tensor $K^{*}$ depends only on $\Lambda^{S}$.

Remark 3.2. The homogenized tensor $K^{*}$ has an extra contribution (26) for its 3,3 entry depending merely on the view factor and not on the cell solutions. It arises from the leakage of the radiative energy at both ends of each cylinder $\Gamma_{\epsilon, i}$ (which are not closed as explained in Remark 2.4). This loss of radiative energy at the cylinders extremities yields this additional axial (or vertical) thermal diffusion. For circular cross-section cylinders (namely $\gamma$ is a circle), we can explicitly compute

$$
\int_{\gamma} \int_{\gamma} F^{2 D}\left(s^{\prime}, y^{\prime}\right)\left|s^{\prime}-y^{\prime}\right|^{2} d y^{\prime} d s^{\prime}=\frac{16}{3} \pi r^{3} \quad \text { where } r \text { is the radius of } \gamma .
$$

If the conductivity tensor $K^{S}$ has a block diagonal structure, namely $K_{3, j}^{S}(x)=K_{j, 3}^{S}(x)=0$ for $j=1,2$, the cell problem (27) has an obvious solution for $j=3$ which yields an explicit formula for $K_{3,3}^{*}$. More precisely, since $P e_{3}=0$ and $\left(I d-\zeta^{2 D}\right) y_{3}=0$, the solution $\omega_{3}^{S}$ is a constant (with respect to $y^{\prime}$ ) for any cell geometry. This implies that $K_{3, j}^{*}(x)=K_{j, 3}^{*}(x)=0$ for $j=1,2$ and

$$
K_{3,3}^{*}(x)=\frac{1}{|\Lambda|}\left(\int_{\Lambda^{S}} K_{3,3}^{S}\left(x, y^{\prime}\right) d y^{\prime}+\frac{\sigma}{2} \int_{\gamma} \int_{\gamma} F^{2 D}\left(s^{\prime}, y^{\prime}\right)\left|s^{\prime}-y^{\prime}\right|^{2} d y^{\prime} d s^{\prime}\right) .
$$

Remark 3.3. As usual in homogenization, Proposition 3.1 gives a complete characterization of the two first terms $T_{0}(x)+\epsilon T_{1}\left(x, \frac{x^{\prime}}{\epsilon}\right)$ of the ansatz (23). With such an approximation, not only do we have a correct estimate of the temperature $T_{\epsilon}(x)$ but also of its gradient (or of the heat flux) since it 
implies $\nabla T_{\epsilon}(x) \approx \nabla T_{0}(x)+\nabla_{y^{\prime}} T_{1}\left(x, \frac{x^{\prime}}{\epsilon}\right)$ (in this last formula the corrector $\nabla_{y^{\prime}} T_{1}$ is of order 1 and can not be ignored).

The proof of Proposition 3.1 shall require the consideration of the second order corrector $T_{2}$ but we are not interested in its precise evaluation since it is much smaller and negligible in the numerical examples (see [4] for a more complete analysis of the second order corrector).

Proof of Proposition 3.1

All the difficulties are concentrated on the radiation term in which simplifications must necessarily take place because it is formally dominating as $\epsilon$ goes to zero. Consequently, instead of using the formal method of two scale asymptotic expansions in the strong form of problem (8), which is complicated because of the non-local boundary condition (the radiation term), we follow the lead of [3] (based on an original idea of J.-L. Lions [26]) and use a two scale asymptotic expansion in the variational formulation of (8), taking advantage of its symmetry. This trick allows us to truncate the ansatz at a lower order term and considerably simplifies the computations.

The variational formulation of problem (8) is: find $T_{\epsilon} \in H_{0}^{1}\left(\Omega_{\epsilon}\right)$ such that

$$
a_{\epsilon}\left(T_{\epsilon}, \phi_{\epsilon}\right)=L_{\epsilon}\left(\phi_{\epsilon}\right) \text { for any } \phi_{\epsilon} \in H_{0}^{1}\left(\Omega_{\epsilon}\right) \text {, }
$$

where

$$
\begin{array}{r}
a_{\epsilon}\left(T_{\epsilon}, \phi_{\epsilon}\right)=\int_{\Omega_{\epsilon}^{S}} K_{\epsilon}^{S}(x) \nabla T_{\epsilon}(x) \cdot \nabla \phi_{\epsilon}(x) d x+\epsilon \int_{\Omega_{\epsilon}^{F}} K_{\epsilon}^{F}(x) \nabla T_{\epsilon}(x) \cdot \nabla \phi_{\epsilon}(x) d x \\
\quad+\int_{\Omega_{\epsilon}^{F}} V_{\epsilon}(x) \cdot \nabla T_{\epsilon}(x) \phi_{\epsilon}(x) d x+\frac{\sigma}{\epsilon} \int_{\Gamma_{\epsilon}} G_{\epsilon}\left(T_{\epsilon}\right)(x) \phi_{\epsilon}(x) d x
\end{array}
$$

and

$$
L_{\epsilon}\left(\phi_{\epsilon}\right)=\int_{\Omega_{\epsilon}^{S}} f(x) \phi_{\epsilon}(x) d x .
$$

We choose $\phi_{\epsilon}$ of the same form as $T_{\epsilon}$ in (23) (but without remainder term)

$$
\phi_{\epsilon}(x)=\phi_{0}(x)+\epsilon \phi_{1}\left(x, \frac{x^{\prime}}{\epsilon}\right)+\epsilon^{2} \phi_{2}\left(x, \frac{x^{\prime}}{\epsilon}\right)
$$

with smooth functions $\phi_{0}(x)$ and $\phi_{i}\left(x, y^{\prime}\right), i=1,2$, being $\Lambda$-periodic in $y^{\prime}$ and such that

$$
\phi_{i}\left(x, y^{\prime}\right)= \begin{cases}\phi_{i}^{S}\left(x, y^{\prime}\right) & \text { in } \Omega \times \Lambda^{S}, \\ \phi_{i}^{F}\left(x, y^{\prime}\right) & \text { in } \Omega \times \Lambda^{F} .\end{cases}
$$

We also assume that $\phi_{0}(x)$ and $\phi_{i}\left(x, y^{\prime}\right)$ have compact support in $x \in \Omega$.

Inserting the ansatz (23) and (30) in the variational formulation (29) yields

$$
a^{0}\left(T_{0}, T_{1}, \phi_{0}, \phi_{1}\right)+\epsilon a^{1}\left(T_{0}, T_{1}, T_{2}, \phi_{0}, \phi_{1}, \phi_{2}\right)=L^{0}\left(\phi_{0}, \phi_{1}\right)+\epsilon L^{1}\left(\phi_{0}, \phi_{1}, \phi_{2}\right)+\mathcal{O}\left(\epsilon^{2}\right) .
$$

The non-conventional strategy of the proof is the following: not only we identify the zero-order term $a^{0}=L^{0}$ but we also use the first-order identity $a^{1}=L^{1}$. The zero-order identity, $a^{0}\left(T_{0}, T_{1}, \phi_{0}, \phi_{1}\right)=$ $L^{0}\left(\phi_{0}, \phi_{1}\right)$, allows us to find the homogenized problem for $T_{0}$ in $\Omega$ and the cell problem for $T_{1}^{S}$ in $\Omega \times \Lambda^{S}$. The first-order identity $a^{1}\left(T_{0}, T_{1}, T_{2}, \phi_{0}, \phi_{1}, \phi_{2}\right)=L^{1}\left(\phi_{0}, \phi_{1}, \phi_{2}\right)$ yields the cell problem for $T_{1}^{F}$ in $\Omega \times \Lambda^{F}$. We emphasize that it is crucial, for the identification of the first-order term, that the test functions $\left(\phi_{i}\right)_{i=0,1,2}$ have compact supports. Indeed, in view of Lemma 2.1, the 3D to $2 \mathrm{D}$ asymptotic of the view factor has a sufficiently small remainder term only for compactly supported test functions.

For the sake of clarity we divide the proof in three steps. The first step is devoted to the ansatz for the convection and diffusion terms. The second one focuses on the radiation term, while the third one 
combines these various terms to deduce the cell and homogenized problems by identifying equations of the same order in powers of $\epsilon$.

We now give the details of the proof. We rewrite the variational formulation (29) as

$$
a_{\epsilon}\left(T_{\epsilon}, \phi_{\epsilon}\right)=a_{\epsilon}^{C}\left(T_{\epsilon}, \phi_{\epsilon}\right)+a_{\epsilon}^{\text {Rad }}\left(T_{\epsilon}, \phi_{\epsilon}\right)=L_{\epsilon}\left(\phi_{\epsilon}\right)
$$

where

$$
\begin{aligned}
a_{\epsilon}^{C}\left(T_{\epsilon}, \phi_{\epsilon}\right)= & \int_{\Omega_{\epsilon}^{S}} K_{\epsilon}^{S}(x) \nabla T_{\epsilon}(x) \cdot \nabla \phi_{\epsilon}(x) d x+\epsilon \int_{\Omega_{\epsilon}^{F}} K_{\epsilon}^{F}(x) \nabla T_{\epsilon}(x) \cdot \nabla \phi_{\epsilon}(x) d x \\
& +\int_{\Omega_{\epsilon}^{F}} V_{\epsilon}(x) \cdot \nabla T_{\epsilon}(x) \phi_{\epsilon}(x) d x \\
a_{\epsilon}^{R a d}\left(T_{\epsilon}, \phi_{\epsilon}\right)= & \frac{\sigma}{\epsilon} \sum_{i=1}^{N(\epsilon)} \int_{\Gamma_{\epsilon, i}} G_{\epsilon}\left(T_{\epsilon}\right)(x) \phi_{\epsilon}(x) d x .
\end{aligned}
$$

\section{Step 1 : Expansion of $a_{\epsilon}^{C}-L_{\epsilon}$}

This is a standard calculation. Plugging the ansatz (23) and (30) we obtain

$$
\begin{aligned}
& a_{\epsilon}^{C}-L_{\epsilon}= \\
& \int_{\Omega_{\epsilon}^{S}} K_{\epsilon}^{S}\left[\left(\nabla_{x} T_{0}+\nabla_{y^{\prime}} T_{1}\right) \cdot\left(\nabla_{x} \phi_{0}+\nabla_{y^{\prime}} \phi_{1}\right)\right]+\int_{\Omega_{\epsilon}^{F}} V_{\epsilon} \cdot\left(\nabla_{x} T_{0}+\nabla_{y^{\prime}} T_{1}\right) \phi_{0} \\
& +\epsilon\left[\int_{\Omega_{\epsilon}^{S}} K_{\epsilon}^{S}\left[\left(\nabla_{x} T_{1}+\nabla_{y^{\prime}} T_{2}\right) \cdot\left(\nabla_{x} \phi_{0}+\nabla_{y^{\prime}} \phi_{1}\right)+\left(\nabla_{x} \phi_{1}+\nabla_{y^{\prime}} \phi_{2}\right) \cdot\left(\nabla_{x} T_{0}+\nabla_{y^{\prime}} T_{1}\right)\right]+\right. \\
& \left.\int_{\Omega_{\epsilon}^{F}} K_{\epsilon}^{F}\left(\nabla_{x} T_{0}+\nabla_{y^{\prime}} T_{1}\right) \cdot\left(\nabla_{x} \phi_{0}+\nabla_{y^{\prime}} \phi_{1}\right)+\int_{\Omega_{\epsilon}^{F}} V_{\epsilon} \cdot\left[\left(\nabla_{x} T_{1}+\nabla_{y^{\prime}} T_{2}\right) \phi_{0}+\left(\nabla_{x} T_{0}+\nabla_{y^{\prime}} T_{1}\right) \phi_{1}\right]\right] \\
& -\int_{\Omega_{\epsilon}^{S}} f\left(\phi_{0}+\epsilon \phi_{1}\right)+\mathcal{O}\left(\epsilon^{2}\right)
\end{aligned}
$$

where all functions are evaluated at $\left(x, x^{\prime} / \epsilon\right)$. Using Lemma 3.2 below, we deduce

$$
\begin{aligned}
& |\Lambda|\left(a_{\epsilon}^{C}-L_{\epsilon}\right)= \\
& \int_{\Omega} \int_{\Lambda^{S}} K^{S}\left(x, y^{\prime}\right)\left(\nabla_{x} T_{0}(x)+\nabla_{y^{\prime}} T_{1}\left(x, y^{\prime}\right) \cdot\left(\nabla_{x} \phi_{0}(x)+\nabla_{y^{\prime}} \phi_{1}\left(x, y^{\prime}\right)\right) d y^{\prime} d x\right. \\
& +\int_{\Omega} \int_{\Lambda^{F}} V\left(x, y^{\prime}\right) \cdot \nabla_{x} T_{0}(x) \phi_{0}(x) d y^{\prime} d x-\int_{\Omega} \int_{\Lambda^{S}} f(x) \phi_{0}(x) d y^{\prime} d x \\
& +\epsilon\left[\int _ { \Omega } \int _ { \Lambda ^ { S } } K ^ { S } ( x , y ^ { \prime } ) \left[\left(\nabla_{x} T_{1}\left(x, y^{\prime}\right)+\nabla_{y^{\prime}} T_{2}\left(x, y^{\prime}\right)\right) \cdot\left(\nabla_{x} \phi_{0}(x)+\nabla_{y^{\prime}} \phi_{1}\left(x, y^{\prime}\right)\right)\right.\right. \\
& \left.\quad+\left(\nabla_{x} \phi_{1}\left(x, y^{\prime}\right)+\nabla_{y^{\prime}} \phi_{2}\left(x, y^{\prime}\right)\right) \cdot\left(\nabla_{x} T_{0}(x)+\nabla_{y^{\prime}} T_{1}\left(x, y^{\prime}\right)\right)\right] d y^{\prime} d x \\
& \quad+\int_{\Omega} \int_{\Lambda^{F}} K^{F}\left(x, y^{\prime}\right)\left(\nabla_{x} T_{0}(x)+\nabla_{y^{\prime}} T_{1}\left(x, y^{\prime}\right)\right) \cdot\left(\nabla_{x} \phi_{0}(x)+\nabla_{y^{\prime}} \phi_{1}\left(x, y^{\prime}\right)\right) d y^{\prime} d x \\
& \quad+\int_{\Omega} \int_{\Lambda^{F}} V\left(x, y^{\prime}\right) \cdot\left[\nabla_{x} T_{1}\left(x, y^{\prime}\right) \phi_{0}(x)+\nabla_{x} T_{0}(x) \phi_{1}\left(x, y^{\prime}\right)+\nabla_{y^{\prime}} T_{1}\left(x, y^{\prime}\right) \phi_{1}\left(x, y^{\prime}\right)\right] d y^{\prime} d x \\
& \left.\quad-\int_{\Omega} \int_{\Lambda^{S}} f(x) \phi_{1}\left(x, y^{\prime}\right) d y^{\prime} d x\right] \\
& +\mathcal{O}\left(\epsilon^{2}\right) .
\end{aligned}
$$




\section{Step 2 : Expansion of $a_{\epsilon}^{R a d}$}

A similar expansion in the 2D setting was carried out in [3]. However, the present 3D configuration is different since, the fluid holes being thin long cylinder, there is also a $3 \mathrm{D}$ to $2 \mathrm{D}$ transition (which did not occur in [3]) taking place. The purpose of this second step is to write a Taylor expansion of the radiation operator, up to second order,

$$
a_{\epsilon}^{\text {Rad }}=a_{0}^{r a d}+\epsilon a_{1}^{r a d}+\mathcal{O}\left(\epsilon^{2}\right)
$$

Fortunately, as we shall see later, the term $a_{1}^{r a d}$ does play any role in the definition of the corrector $T_{1}$ in $\Lambda^{F}$. Therefore, we don't need to evaluate $a_{1}^{r a d}$ which, of course, significantly reduces the amount of tedious calculations. The radiation term is given by

$$
a_{\epsilon}^{R a d}=\frac{\sigma}{\epsilon} \sum_{i=1}^{N(\epsilon)}\left[\int_{\Gamma_{\epsilon, i}} T_{\epsilon}(x) \phi_{\epsilon}(x) d x-\int_{\Gamma_{\epsilon, i}} \int_{\Gamma_{\epsilon, i}} F(x, s) T_{\epsilon}(x) \phi_{\epsilon}(s) d x d s\right] .
$$

In the ansatz (23) and (30) we make a Taylor expansion around each center of mass $x_{0, i}^{\prime}$ of each boundary $\gamma_{\epsilon, i}$. To simplify the notations, we drop the label $i$ and denote by $x_{0}^{\prime}$ each $x_{0, i}^{\prime}$. We also denote $\left(x^{\prime}-x_{0}^{\prime}\right)$ by $\epsilon h^{\prime}$ and $\left(s^{\prime}-x_{0}^{\prime}\right)$ by $\epsilon l^{\prime}$. Thus we get

$$
\begin{aligned}
& T_{\epsilon}(x)=T_{0}\left(x_{0}^{\prime}, x_{3}\right)+\epsilon\left(\nabla_{x^{\prime}} T_{0}\left(x_{0}^{\prime}, x_{3}\right) \cdot h^{\prime}+T_{1}\left(x_{0}^{\prime}, \frac{x^{\prime}}{\epsilon}, x_{3}\right)\right)+\epsilon^{2} \widehat{T}_{2, \epsilon}(x)+\mathcal{O}\left(\epsilon^{3}\right) \\
& \phi_{\epsilon}(s)=\phi_{0}\left(x_{0}^{\prime}, s_{3}\right)+\epsilon\left(\nabla_{x^{\prime}} \phi_{0}\left(x_{0}^{\prime}, s_{3}\right) \cdot l^{\prime}+\phi_{1}\left(x_{0}^{\prime}, \frac{s^{\prime}}{\epsilon}, s_{3}\right)\right)+\epsilon^{2} \widehat{\phi}_{2, \epsilon}(s)+\mathcal{O}\left(\epsilon^{3}\right)
\end{aligned}
$$

where

$$
\begin{gathered}
\widehat{T}_{2, \epsilon}(x)=\frac{1}{2} \nabla_{x^{\prime}} \nabla_{x^{\prime}} T_{0}\left(x_{0}^{\prime}, x_{3}\right) h^{\prime} \cdot h^{\prime}+\nabla_{x^{\prime}} T_{1}\left(x_{0}^{\prime}, \frac{x^{\prime}}{\epsilon}, x_{3}\right) \cdot h^{\prime}+T_{2}\left(x_{0}^{\prime}, \frac{x^{\prime}}{\epsilon}, x_{3}\right) \\
\widehat{\phi}_{2, \epsilon}(s)=\frac{1}{2} \nabla_{x^{\prime}} \nabla_{x^{\prime}} \phi_{0}\left(x_{0}^{\prime}, s_{3}\right) l^{\prime} \cdot l^{\prime}+\nabla_{x^{\prime}} \phi_{1}\left(x_{0}^{\prime}, \frac{s^{\prime}}{\epsilon}, s_{3}\right) \cdot l^{\prime}+\phi_{2}\left(x_{0}^{\prime}, \frac{s^{\prime}}{\epsilon}, s_{3}\right)
\end{gathered}
$$

The precise form of the terms $\widehat{T}_{2, \epsilon}$ and $\widehat{\phi}_{2, \epsilon}$ is not important since the $\mathcal{O}\left(\epsilon^{2}\right)$-order terms will disappear by simplification as we shall see later. Using (37) and (38), we obtain

$$
T_{\epsilon}(x) \phi_{\epsilon}(s)=(T \phi)_{0}\left(x_{3}, s_{3}\right)+\epsilon(T \phi)_{1}(x, s)+\epsilon^{2}(T \phi)_{2}(x, s)+\mathcal{O}\left(\epsilon^{3}\right) .
$$

where

$$
\begin{aligned}
(T \phi)_{0}\left(x_{3}, s_{3}\right) & =\phi_{0}\left(x_{0}^{\prime}, s_{3}\right) T_{0}\left(x_{0}^{\prime}, x_{3}\right) \\
(T \phi)_{1}(x, s) & =\phi_{0}\left(x_{0}^{\prime}, s_{3}\right) \nabla_{x^{\prime}} T_{0}\left(x_{0}^{\prime}, x_{3}\right) \cdot h^{\prime}+T_{0}\left(x_{0}^{\prime}, x_{3}\right) \nabla_{x^{\prime}} \phi_{0}\left(x_{0}^{\prime}, s_{3}\right) \cdot l^{\prime} \\
& +\phi_{0}\left(x_{0}^{\prime}, s_{3}\right) T_{1}\left(x_{0}^{\prime}, \frac{x^{\prime}}{\epsilon}, x_{3}\right)+\phi_{1}\left(x_{0}^{\prime}, \frac{s^{\prime}}{\epsilon}, s_{3}\right) T_{0}\left(x_{0}^{\prime}, x_{3}\right) \\
(T \phi)_{2}(x, s) & =\phi_{1}\left(x_{0}^{\prime}, \frac{s^{\prime}}{\epsilon}, s_{3}\right) T_{1}\left(x_{0}^{\prime}, \frac{x^{\prime}}{\epsilon}, x_{3}\right)+\widehat{T}_{2, \epsilon} \phi_{0}\left(x_{0}^{\prime}, s_{3}\right)+\widehat{\phi}_{2, \epsilon} T_{0}\left(x_{0}^{\prime}, x_{3}\right) \\
& +\nabla_{x^{\prime}} \phi_{0}\left(x_{0}^{\prime}, s_{3}\right) \cdot l^{\prime} \nabla_{x^{\prime}} T_{0}\left(x_{0}^{\prime}, s_{3}\right) \cdot h^{\prime}+\phi_{1}\left(x_{0}^{\prime}, \frac{s^{\prime}}{\epsilon}, s_{3}\right) \nabla_{x^{\prime}} T_{0}\left(x_{0}^{\prime}, s_{3}\right) \cdot h^{\prime} \\
& +T_{1}\left(x_{0}^{\prime}, \frac{x^{\prime}}{\epsilon}, x_{3}\right) \nabla_{x^{\prime}} \phi_{0}\left(x_{0}^{\prime}, s_{3}\right) \cdot l^{\prime}
\end{aligned}
$$


Since the test functions $\phi_{i}$ have compact support in $\Omega$, we can use formula (16) of Lemma 2.1 (or formula (22) of Remark 2.5) for the 3D to 2D asymptotic behavior of the view factor. Thus we deduce

$$
\begin{gathered}
\int_{\Gamma_{\epsilon, i}} \int_{\Gamma_{\epsilon, i}}(T \phi)_{0}\left(x_{3}, s_{3}\right) F(s, x) d s d x=\int_{\gamma_{\epsilon, i}} \int_{\gamma_{\epsilon, i}} F^{2 D}\left(s^{\prime}, x^{\prime}\right) \int_{0}^{L_{3}}(T \phi)_{0}\left(x_{3}, x_{3}\right) d x_{3} \\
-\frac{1}{2} \int_{\gamma_{\epsilon, i}} \int_{\gamma_{\epsilon, i}} F^{2 D}\left(s^{\prime}, x^{\prime}\right)\left|x^{\prime}-s^{\prime}\right|^{2} \int_{0}^{L_{3}} \frac{\partial \phi_{0}}{\partial x_{3}}\left(x_{0}^{\prime}, x_{3}\right) \frac{\partial T_{0}}{\partial x_{3}}\left(x_{0}^{\prime}, x_{3}\right) d x_{3}+\left|\gamma_{\epsilon, i}\right|^{2} \mathcal{O}\left(\epsilon^{p-1}\right),
\end{gathered}
$$

with $0<p<3$. Then, since $\left|\gamma_{\epsilon, i}\right|=\epsilon|\gamma|$,

$$
\begin{aligned}
& \frac{1}{\epsilon}\left(\int_{\Gamma_{\epsilon, i}}(T \phi)_{0}\left(x_{3}, x_{3}\right) d x-\int_{\Gamma_{\epsilon, i}} \int_{\Gamma_{\epsilon, i}}(T \phi)_{0}\left(x_{3}, s_{3}\right) F(s, x) d s d x\right) \\
& \quad=\frac{1}{2 \epsilon} \int_{\gamma_{\epsilon, i}} \int_{\gamma_{\epsilon, i}} F^{2 D}\left(s^{\prime}, x^{\prime}\right)\left|x^{\prime}-s^{\prime}\right|^{2} \int_{0}^{L_{3}} \frac{\partial \phi_{0}}{\partial x_{3}}\left(x_{0}^{\prime}, x_{3}\right) \frac{\partial T_{0}}{\partial x_{3}}\left(x_{0}^{\prime}, x_{3}\right) d x_{3}+|\gamma|^{2} \mathcal{O}\left(\epsilon^{p}\right) .
\end{aligned}
$$

A similar computation, taking into account the various symmetry properties of the view factor, yields

$$
\frac{1}{\epsilon}\left(\int_{\Gamma_{\epsilon, i}} \epsilon(T \phi)_{1}(x, x) d x-\int_{\Gamma_{\epsilon, i}} \int_{\Gamma_{\epsilon, i}} \epsilon(T \phi)_{1}(x, s) F(s, x) d s d x\right)=\mathcal{O}\left(\epsilon^{3}\right),
$$

and

$$
\begin{aligned}
\frac{1}{\epsilon} & \left(\int_{\Gamma_{\epsilon, i}} \epsilon^{2}(T \phi)_{2}(x, x) d x-\int_{\Gamma_{\epsilon, i}} \int_{\Gamma_{\epsilon, i}} \epsilon^{2}(T \phi)_{2}(x, s) F(s, x) d s d x\right) \\
& =\epsilon\left(\int_{0}^{L_{3}} \int_{\gamma_{\epsilon, i}} \phi_{1}\left(x_{0}^{\prime}, \frac{s^{\prime}}{\epsilon}, x_{3}\right)\left[T_{1}\left(x_{0}^{\prime}, \frac{s^{\prime}}{\epsilon}, x_{3}\right)-\int_{\gamma_{\epsilon, i}} T_{1}\left(x_{0}^{\prime}, \frac{x^{\prime}}{\epsilon}, x_{3}\right) F^{2 D}\left(s^{\prime}, x^{\prime}\right) d x^{\prime}\right] d s^{\prime} d x_{3}\right. \\
& +\int_{0}^{L_{3}} \int_{\gamma_{\epsilon, i}}\left(\nabla_{x^{\prime}} \phi_{0}\left(x_{0}^{\prime}, x_{3}\right) \cdot l^{\prime}\right) \nabla_{x^{\prime}} T_{0}\left(x_{0}^{\prime}, x_{3}\right) \cdot\left[l^{\prime}-\int_{\gamma_{\epsilon, i}} h^{\prime} F^{2 D}\left(s^{\prime}, x^{\prime}\right) d x^{\prime}\right] d s^{\prime} d x_{3} \\
& +\int_{0}^{L_{3}} \int_{\gamma_{\epsilon, i}} \nabla_{x^{\prime}} \phi_{0}\left(x_{0}^{\prime}, x_{3}\right) \cdot l^{\prime}\left[T_{1}\left(x_{0}^{\prime}, \frac{s^{\prime}}{\epsilon}, x_{3}\right)-\int_{\gamma_{\epsilon, i}} T_{1}\left(x_{0}^{\prime}, \frac{x^{\prime}}{\epsilon}, x_{3}\right) F^{2 D}\left(s^{\prime}, x^{\prime}\right) d x^{\prime}\right] d s^{\prime} d x_{3} \\
& \left.+\int_{0}^{L_{3}} \int_{\gamma_{\epsilon, i}} \phi_{1}\left(x_{0}^{\prime}, \frac{s^{\prime}}{\epsilon}, x_{3}\right) \nabla_{x^{\prime}} T_{0}\left(x_{0}^{\prime}, x_{3}\right) \cdot\left[l^{\prime}-\int_{\gamma_{\epsilon, i}} h^{\prime} F^{2 D}\left(s^{\prime}, x^{\prime}\right) d x^{\prime}\right] d s^{\prime} d x_{3}\right)+\mathcal{O}\left(\epsilon^{3}\right) .
\end{aligned}
$$

In (40) and (41), we do not give the explicit form of the remainder terms (including the diffusive term coming from the 3D to $2 \mathrm{D}$ limit in the view factor) which are negligible after rescaling and summation over all cells as soon as they are of order $\mathcal{O}\left(\epsilon^{q}\right)$ with $q>2$.

Thus Lemma 3.2, the changes of variables $y^{\prime}=x^{\prime} / \epsilon$ and $z^{\prime}=s^{\prime} / \epsilon$ in (39), (40), (41) and summing over all cells, yield

$$
\frac{\sigma}{\epsilon}\left[\sum_{i=1}^{N(\epsilon)} \int_{\Gamma_{\epsilon, i}} T_{\epsilon}(x) \phi_{\epsilon}(x) d x-\int_{\Gamma_{\epsilon, i}} \int_{\Gamma_{\epsilon, i}} T_{\epsilon}(x) \phi_{\epsilon}(s) F(s, x) d s d x\right]=a_{0}^{r a d}+\mathcal{O}\left(\epsilon^{p-2}\right)
$$


with

$$
\begin{aligned}
a_{0}^{r a d} & =\frac{\sigma}{|\Lambda|}\left(\frac{1}{2} \int_{\Omega} \frac{\partial \phi_{0}}{\partial x_{3}}(x) \frac{\partial T_{0}}{\partial x_{3}}(x) d x \int_{\gamma} \int_{\gamma} F^{2 D}\left(z^{\prime}, y^{\prime}\right)\left|z^{\prime}-y^{\prime}\right|^{2} d y^{\prime} d z^{\prime}\right. \\
& +\int_{\Omega} \nabla_{x^{\prime}} \phi_{0}(x) \cdot \int_{\gamma}\left(y^{\prime} \otimes y^{\prime}-\int_{\gamma} y^{\prime} \otimes z^{\prime} F^{2 D}\left(z^{\prime}, y^{\prime}\right) d z^{\prime}\right) d y^{\prime} \nabla_{x^{\prime}} T_{0}(x) d x \\
& +\int_{\Omega} \nabla_{x^{\prime}} T_{0}(x) \cdot \int_{\gamma} \phi_{1}\left(x, y^{\prime}\right)\left(y^{\prime}-\int_{\gamma} z^{\prime} F^{2 D}\left(z^{\prime}, y^{\prime}\right) d z^{\prime}\right) d y^{\prime} d x \\
& +\int_{\Omega} \nabla_{x^{\prime}} \phi_{0}(x) \cdot \int_{\gamma} T_{1}\left(x, y^{\prime}\right)\left(y^{\prime}-\int_{\gamma} z^{\prime} F^{2 D}\left(z^{\prime}, y^{\prime}\right) d z^{\prime}\right) d y^{\prime} d x \\
& \left.+\int_{\Omega} \int_{\gamma} \int_{\gamma}\left(\delta\left(y^{\prime}-z^{\prime}\right)-F^{2 D}\left(y^{\prime}, z^{\prime}\right)\right) T_{1}\left(x, z^{\prime}\right) \phi_{1}\left(x, y^{\prime}\right) d z^{\prime} d y^{\prime} d x\right)
\end{aligned}
$$

where $\delta$ is the Dirac mass. Remark that the last term in (43) can also be written

$$
\int_{\gamma} \int_{\gamma}\left(\delta\left(y^{\prime}-z^{\prime}\right)-F^{2 D}\left(y^{\prime}, z^{\prime}\right)\right) T_{1}\left(x, z^{\prime}\right) \phi_{1}\left(x, y^{\prime}\right) d z^{\prime} d y^{\prime}=\int_{\gamma} \phi_{1}\left(x, y^{\prime}\right)\left(\left(I d-\zeta^{2 D}\right) T_{1}\right)\left(x, y^{\prime}\right) d y^{\prime} .
$$

Remark 3.4. As already said, in the spirit of our proof we should also compute the next order term $a_{1}^{\text {rad }}$ in the asymptotic expansion $a_{\epsilon}^{\text {Rad }}=a_{0}^{\text {rad }}+\epsilon a_{1}^{\text {rad }}+\mathcal{O}\left(\epsilon^{2}\right)$. The computation of $a_{1}^{\text {rad }}$ is tedious and require to carry the expansions of $T_{\epsilon}$ and $\phi_{\epsilon}$ to one more order in $\epsilon$, a formidable task that is not pursued here (similar computations can be found in [4] for a 2D-configuration). Fortunately, the radiation term contributes merely to the boundary condition for the cell problem in the solid part $\Lambda^{S}$ and does not play any role for the cell problem in the fluid part $\Lambda^{F}$. Since the first-order terms $a^{1}, L^{1}$ are used to deduce the fluid cell problem, it is perfectly legitimate not to compute $a_{1}^{\text {rad }}$.

\section{Step 3 : Identification of the limit variational formulations}

The zero-th order $\epsilon^{0}$-term of (31) is

$$
a^{0}\left(T_{0}, T_{1}, \phi_{0}, \phi_{1}\right)=L^{0}\left(\phi_{0}, \phi_{1}\right)
$$

which is equivalent to

$$
\begin{aligned}
& \int_{\Omega} \int_{\Lambda^{S}} K^{S}\left(x, y^{\prime}\right)\left(\nabla_{x} T_{0}(x)+\nabla_{y^{\prime}} T_{1}\left(x, y^{\prime}\right) \cdot\left(\nabla_{x} \phi_{0}(x)+\nabla_{y^{\prime}} \phi_{1}\left(x, y^{\prime}\right)\right) d y^{\prime} d x\right. \\
+ & \int_{\Omega} \int_{\Lambda^{F}} V\left(x, y^{\prime}\right) \cdot \nabla_{x} T_{0}(x) \phi_{0}(x) d y^{\prime} d x \\
+ & \frac{\sigma}{2} \int_{\Omega} \frac{\partial \phi_{0}}{\partial x_{3}}(x) \frac{\partial T_{0}}{\partial x_{3}}(x) d x \int_{\gamma} \int_{\gamma} F^{2 D}\left(z^{\prime}, y^{\prime}\right)\left|z^{\prime}-y^{\prime}\right|^{2} d y^{\prime} d z^{\prime} \\
+ & \sigma \int_{\Omega} \nabla_{x^{\prime}} T_{0}(x) \cdot \int_{\gamma} \phi_{1}\left(x, y^{\prime}\right)\left(y^{\prime}-\int_{\gamma} z^{\prime} F^{2 D}\left(z^{\prime}, y^{\prime}\right) d z^{\prime}\right) d y^{\prime} d x \\
+ & \sigma \int_{\Omega} \nabla_{x^{\prime}} \phi_{0}(x) \cdot \int_{\gamma} T_{1}\left(x, y^{\prime}\right)\left(y^{\prime}-\int_{\gamma} z^{\prime} F^{2 D}\left(z^{\prime}, y^{\prime}\right) d z^{\prime}\right) d y^{\prime} d x \\
+ & \sigma \int_{\Omega} \nabla_{x^{\prime}} \phi_{0}(x) \cdot \int_{\gamma}\left(y^{\prime} \otimes y^{\prime}-\int_{\gamma} y^{\prime} \otimes z^{\prime} F^{2 D}\left(z^{\prime}, y^{\prime}\right) d z^{\prime}\right) \nabla_{x^{\prime}} T_{0}(x) d y^{\prime} d x \\
+ & \sigma \int_{\Omega} \int_{\gamma} \int_{\gamma}\left(\delta\left(y^{\prime}-z^{\prime}\right)-F^{2 D}\left(y^{\prime}, z^{\prime}\right)\right) T_{1}\left(x, z^{\prime}\right) \phi_{1}\left(x, y^{\prime}\right) d z^{\prime} d y^{\prime} d x \\
= & \left|\Lambda^{S}\right| \int_{\Omega} f(x) \phi_{0}(x) d x
\end{aligned}
$$


We recognize in (44) the variational formulation of the so-called two-scale limit problem which is a combination of the homogenized and cell problems (in $\Lambda^{S}$ only).

We recover the cell problem in $\Lambda^{S}$ by taking $\phi_{0}=0$ in the limit of the variational formulation (44)

$$
\begin{array}{r}
\int_{\Omega} \int_{\Lambda^{S}} K^{S}\left(x, y^{\prime}\right)\left(\nabla_{x} T_{0}(x)+\nabla_{y^{\prime}} T_{1}\left(x, y^{\prime}\right)\right) \cdot \nabla_{y^{\prime}} \phi_{1}\left(x, y^{\prime}\right) d y^{\prime} d x \\
+\sigma \int_{\Omega} \nabla_{x^{\prime}} T_{0}(x) \cdot \int_{\gamma} \phi_{1}\left(x, y^{\prime}\right)\left(y^{\prime}-\int_{\gamma} z^{\prime} F^{2 D}\left(z^{\prime}, y^{\prime}\right) d z^{\prime}\right) d y^{\prime} d x \\
+\sigma \int_{\Omega} \int_{\gamma} \int_{\gamma}\left(\delta\left(y^{\prime}-z^{\prime}\right)-F^{2 D}\left(y^{\prime}, z^{\prime}\right)\right) T_{1}\left(x, z^{\prime}\right) \phi_{1}\left(x, y^{\prime}\right) d z^{\prime} d y^{\prime} d x=0
\end{array}
$$

The solution $T_{1}$ of the above variational formulation is given by (28) in $\Lambda^{S}$ where $\omega_{j} \equiv \omega_{j}^{S}\left(x, y^{\prime}\right)$, for $1 \leq j \leq 3$, are the solutions of the cell problems in the $2 \mathrm{D}$ solid media $\Lambda^{S}$

$$
\left\{\begin{aligned}
-\operatorname{div}_{y^{\prime}} P\left[K^{S}\left(x, y^{\prime}\right)\left(e_{j}+\nabla_{y^{\prime}} \omega_{j}^{S}\left(y^{\prime}\right)\right)\right] & =0 & & \text { in } \Lambda^{S} \\
-P\left[K^{S}\left(x, y^{\prime}\right)\left(e_{j}+\nabla_{y^{\prime}} \omega_{j}^{S}\left(y^{\prime}\right)\right)\right] \cdot n & =\sigma\left(I d-\zeta^{2 D}\right)\left(\omega_{j}^{S}\left(y^{\prime}\right)+y_{j}\right) & & \text { on } \gamma \\
y^{\prime} & \mapsto \omega_{j}^{S}\left(y^{\prime}\right) & & \text { is } \Lambda \text {-periodic. }
\end{aligned}\right.
$$

Remark 3.5. As already said, the macroscopic variable $x$ plays the role of a parameter in (46). Therefore, for the sake of notational simplicity we shall often forget the dependence on $x$ for the solutions $\omega_{j}$ of the cell problems.

To recover the homogenized problem we now substitute $\phi_{1}$ by 0 in (44). We obtain

$$
\begin{aligned}
& \int_{\Omega} \int_{\Lambda^{S}} \sum_{k, j=1}^{3} K^{S}\left(x, y^{\prime}\right)\left(\nabla_{y^{\prime}} \omega_{k}\left(y^{\prime}\right)+e_{k}\right) \cdot\left(\nabla_{y^{\prime}} \omega_{j}\left(y^{\prime}\right)+e_{j}\right) \frac{\partial T_{0}}{\partial x_{k}}(x) \frac{\partial \phi_{0}}{\partial x_{j}}(x) d y^{\prime} d x \\
+ & \int_{\Omega} \int_{\Lambda^{F}} \sum_{k=1}^{3} V_{K}\left(x, y^{\prime}\right) \frac{\partial T_{0}}{\partial x_{k}}(x) \phi_{0}(x) d y^{\prime} d x \\
+ & \frac{\sigma}{2} \int_{\Omega} \frac{\partial \phi_{0}}{\partial x_{3}}(x) \frac{\partial T_{0}}{\partial x_{3}}(x) d x \int_{\gamma} \int_{\gamma} F^{2 D}\left(z^{\prime}, y^{\prime}\right)\left|z^{\prime}-y^{\prime}\right|^{2} d y^{\prime} d z^{\prime} \\
+ & \sigma \int_{\Omega} \int_{\gamma} \sum_{k, j=1}^{3}\left(I d-\zeta^{2 D}\right)\left(\omega_{k}\left(y^{\prime}\right)+y_{k}\right)\left(\omega_{j}\left(y^{\prime}\right)+y_{j}\right) \frac{\partial T_{0}}{\partial x_{k}}(x) \frac{\partial \phi_{0}}{\partial x_{j}}(x) d y^{\prime} d x \\
= & \left|\Lambda^{S}\right| \int_{\Omega} f(x) \phi_{0}(x) d x
\end{aligned}
$$

which is the variational formulation of the homogenized problem (25) where $K^{*}$ and $V^{*}$ are given by the formulas of Proposition 3.1.

We now turn to the first order $\epsilon^{1}$-term of (31) which yields the cell problem in $\Lambda^{F}$. Indeed, up to this point, the zero-th order term of (31) has given the cell problem in $\Lambda^{S}$, as well as the homogenized problem for $T_{0}$ in the domain $\Omega$. Nonetheless, as we already said in Remark 3.3, we want to compute everywhere the corrector $T_{1}$ of the solution $T_{\epsilon}$, not merely in the solid part. Therefore, we look at the next, $\epsilon^{1}$-order term of $(31)$

$$
a^{1}\left(T_{0}, T_{1}, T_{2}, \phi_{0}, \phi_{1}, \phi_{2}\right)=L^{1}\left(\phi_{0}, \phi_{1}, \phi_{2}\right)
$$


where we shall keep only the terms coming from the fluid part (those coming from the solid part will contribute to the determination of $T_{2}$ which we do not pursue here). It is equivalent to

$$
\begin{aligned}
& \int_{\Omega} \int_{\Lambda^{S}} K^{S}\left(x, y^{\prime}\right)\left[\left(\nabla_{x} T_{1}\left(x, y^{\prime}\right)+\nabla_{y^{\prime}} T_{2}\left(x, y^{\prime}\right)\right) \cdot\left(\nabla_{x} \phi_{0}(x)+\nabla_{y^{\prime}} \phi_{1}\left(x, y^{\prime}\right)\right)\right. \\
+ & \left.\left(\nabla_{x} \phi_{1}\left(x, y^{\prime}\right)+\nabla_{y^{\prime}} \phi_{2}\left(x, y^{\prime}\right)\right) \cdot\left(\nabla_{x} T_{0}(x)+\nabla_{y^{\prime}} T_{1}\left(x, y^{\prime}\right)\right)\right] d y^{\prime} d x \\
+ & \int_{\Omega} \int_{\Lambda^{F}} K^{F}\left(x, y^{\prime}\right)\left(\nabla_{x} T_{0}(x)+\nabla_{y^{\prime}} T_{1}\left(x, y^{\prime}\right)\right) \cdot\left(\nabla_{x} \phi_{0}(x)+\nabla_{y^{\prime}} \phi_{1}\left(x, y^{\prime}\right)\right) d y^{\prime} d x \\
+ & \int_{\Omega} \int_{\Lambda^{F}} V\left(x, y^{\prime}\right) \cdot\left(\nabla_{x} T_{1}\left(x, y^{\prime}\right) \phi_{0}(x)+\nabla_{x} T_{0}(x) \phi_{1}\left(x, y^{\prime}\right)+\nabla_{y^{\prime}} T_{1}\left(x, y^{\prime}\right) \phi_{1}\left(x, y^{\prime}\right)\right) d y^{\prime} d x \\
+ & a_{1}^{r a d} \\
= & \int_{\Omega} \int_{\Lambda^{S}} f(x) \phi_{1}\left(x, y^{\prime}\right) d y^{\prime} d x
\end{aligned}
$$

Note that, by virtue of Lemma 3.2, the approximation of an integral on $\Omega_{\epsilon}^{S, F}$ by a double integral on $\Omega \times \Lambda^{S, F}$ is of order $\epsilon^{2}$ and thus does not interact with the first order $\epsilon^{1}$-term of (31).

In (48) we take $\phi_{0} \equiv 0$ and $\phi_{2} \equiv 0$ everywhere, and $\phi_{1}=0$ in $\Lambda^{S}$ only. It thus becomes the variational formulation of

$$
\left\{\begin{array}{c}
-\operatorname{div}_{y^{\prime}} P\left[K^{F}\left(x, y^{\prime}\right)\left(\nabla T_{0}+\nabla_{y^{\prime}} T_{1}\right)\right]+V\left(x, y^{\prime}\right) \cdot\left(\nabla_{x} T_{0}+\nabla_{y^{\prime}} T_{1}\right)=0 \quad \text { in } \Lambda^{F}, \\
T_{1} \text { is continuous through } \gamma .
\end{array}\right.
$$

Therefore, the solution $T_{1}$ of (49) is given by (28) in $\Lambda^{F}$ where $\omega_{j} \equiv \omega_{j}^{F}\left(x, y^{\prime}\right)$, for $1 \leq j \leq 3$, are the solutions of the cell problems in $\Lambda^{F}$

$$
\left\{\begin{array}{rrr}
-\operatorname{div}_{y^{\prime}} P\left[K^{F}\left(x, y^{\prime}\right)\left(e_{j}+\nabla_{y^{\prime}} \omega_{j}^{F}\left(y^{\prime}\right)\right)\right]+V\left(x, y^{\prime}\right) \cdot\left(e_{j}+\nabla_{y^{\prime}} \omega_{j}^{F}\left(y^{\prime}\right)\right) & =0 & \text { in } \Lambda^{F} \\
\omega_{j}^{F} & =\omega_{j}^{S} & \text { on } \gamma \\
y^{\prime} \mapsto \omega_{j}^{F}\left(y^{\prime}\right) & \text { is } \Lambda \text {-periodic. }
\end{array}\right.
$$

Combining (46) and (50), we get (27).

Lemma 3.1. Each of the cell problems (27) admits a unique solution, up to a constant, in $H_{\#}^{1}(\Lambda)$.

Proof First, we recall that each cell problem in $\Lambda$ is decoupled into two independents cell problems, (46) in $\Lambda^{S}$ with a radiative boundary condition and (50) in $\Lambda^{F}$ with a Dirichlet boundary condition.

For $\phi \in H_{\#}^{1}\left(\Lambda^{S}\right)$, the variational formulation of (46) is given by

$$
\int_{\Lambda^{S}} K^{S}\left(x, y^{\prime}\right)\left(\nabla_{y^{\prime}} \omega_{i}^{S}\left(y^{\prime}\right)+e_{i}\right) \cdot \nabla_{y^{\prime}} \phi\left(y^{\prime}\right)+\sigma \int_{\gamma}\left(I d-\zeta^{2 D}\right)\left(\omega_{i}^{S}\left(y^{\prime}\right)+y_{i}^{\prime}\right) \phi\left(y^{\prime}\right)=0 .
$$

Using (11), we deduce that the bilinear form of $(51)$ is coercive on $H_{\#}^{1}\left(\Lambda^{S}\right) / \mathbb{R}$

$$
a(\phi, \phi)=\int_{\Lambda^{S}} K^{S} \nabla_{y^{\prime}} \phi \cdot \nabla_{y^{\prime}} \phi+\sigma \int_{\gamma}\left(I d-\zeta^{2 D}\right) \phi \phi \geq C\left\|\nabla_{y} \phi\right\|_{L_{\#}^{2}\left(\Lambda^{S}\right)} \equiv C\|\phi\|_{H_{\#}^{1}\left(\Lambda^{S}\right) / \mathbb{R}} .
$$

Furthermore, since (12) implies that (51) holds true when the test function $\phi$ is a constant, the Fredholm alternative (see [31]) yields existence and uniqueness in $H_{\#}^{1}\left(\Lambda^{S}\right) / \mathbb{R}$ (i.e., up to a constant) of the cell problem (46) solution.

The existence of a unique solution in $H_{\#}^{1}\left(\Lambda^{F}\right)$ of the fluid cell problems (50), with a non-homogeneous Dirichlet boundary condition, is completely standard for this simple convection-diffusion equation (note that, for our geometry in Figure 2, the periodic boundary condition does not appear in the fluid cell $\Lambda^{F}$ ). Of course, since $\omega_{j}^{S}$ is defined up to a constant, so is $\omega_{j}^{F}$, but with the same constant. 
We recall a classical lemma used in the proof of Proposition 3.1.

Lemma 3.2. For a smooth function $f$ and any integer $p \geq 0$ we have

$$
\begin{array}{rlrl}
\text { i. } \int_{\gamma_{\epsilon, i}} f\left(\frac{x^{\prime}}{\epsilon}\right)\left(x_{k}^{\prime}-x_{0, k}^{\prime}\right)^{p} d x^{\prime} & =\epsilon^{1+p} \int_{\gamma} f\left(y^{\prime}\right)\left(y_{k}^{\prime}-y_{0, k}^{\prime}\right)^{p} d y^{\prime} \\
\text { ii. } \quad \epsilon^{2} \sum_{i=1}^{N(\epsilon)} \int_{0}^{L_{3}} f\left(x_{0, i}^{\prime}, x_{3}\right) d x_{3} & =\frac{1}{|\Lambda|} \int_{\Omega} f(x) d x+\mathcal{O}\left(\epsilon^{2}\right) \\
\text { iii. } & \int_{\Omega_{\epsilon}^{S, F}} f\left(x, \frac{x^{\prime}}{\epsilon}\right) d x & =\frac{1}{|\Lambda|} \int_{\Omega} \int_{\Lambda^{S, F}} f\left(x, y^{\prime}\right) d y^{\prime} d x+\mathcal{O}\left(\epsilon^{2}\right)
\end{array}
$$

Proof The first formula is immediate by a simple change of variables. For the second one, we perform a Taylor expansion of $f\left(x^{\prime}\right)$ (which is assumed to be $C^{2}$ ) around $x_{0, i}^{\prime}$ the center of mass of each cell $\Lambda_{\epsilon, i}$

$$
f\left(x^{\prime}, x_{3}\right)=f\left(x_{0, i}^{\prime}, x_{3}\right)+\left(x^{\prime}-x_{0, i}^{\prime}\right) \nabla_{x^{\prime}} f\left(x_{0, i}^{\prime}, x_{3}\right)+\mathcal{O}\left(\epsilon^{2}\right)
$$

which becomes by integration in $\Lambda_{\epsilon, i}$

$$
\int_{\Lambda_{\epsilon, i}} f\left(x^{\prime}, x_{3}\right) d x^{\prime}=\epsilon^{2}|\Lambda| f\left(x_{0, i}^{\prime}, x_{3}\right)+\mathcal{O}\left(\epsilon^{4}\right)
$$

because $\left|\Lambda_{\epsilon, i}\right|=\epsilon^{2}|\Lambda|$ and $\int_{\Lambda_{\epsilon, i}}\left(x^{\prime}-x_{0, i}^{\prime}\right) d x^{\prime}=0$. After summation, and integration between 0 and $L_{3}$ in $x_{3}$, we obtain the desired result. The third formula is a consequence of the Riemann-Lebesgue lemma which states that the integral of a periodically oscillating smooth function converges to its average with a convergence speed smaller than any power of $\epsilon$ (this is obtained by repeated integration by parts since any zero-average periodic function of the fast $y$ variable is the divergence of a zero-average periodic vector field in $y$ ).

\section{Convergence}

The results of the previous section are only formal. They are based on the assumption that the temperature $T_{\epsilon}$ admits the asymptotic expansion (23). Therefore, to complete our study, we need a rigorous mathematical justification of Proposition 3.1. Here, we prove a convergence result using the two-scale convergence method [1], [30].

\subsection{A priori estimates}

To use the two-scale convergence method, we first need to establish some a priori estimates on the unknown $T_{\epsilon}$.

Proposition 4.1. Let $T_{\epsilon}$ be the solution of problem (8). There exists a constant $C$, not depending on $\epsilon$, such that

$$
\left\|T_{\epsilon}\right\|_{L^{2}(\Omega)}+\left\|\nabla T_{\epsilon}\right\|_{L^{2}\left(\Omega_{\epsilon}^{S}\right)}+\sqrt{\epsilon}\left\|\nabla T_{\epsilon}\right\|_{L^{2}\left(\Omega_{\epsilon}^{F}\right)}+\sqrt{\epsilon}\left\|T_{\epsilon}\right\|_{L^{2}\left(\Gamma_{\epsilon}\right)} \leq C
$$

Proof Taking $\phi_{\epsilon}=T_{\epsilon}$ in the variational formulation (29) of (8) we obtain

$$
\int_{\Omega_{\epsilon}^{S}} K_{\epsilon}^{S}\left|\nabla T_{\epsilon}\right|^{2} d x+\epsilon \int_{\Omega_{\epsilon}^{F}} K_{\epsilon}^{F}\left|\nabla T_{\epsilon}\right|^{2} d x+\int_{\Omega_{\epsilon}^{F}} V_{\epsilon} \cdot \nabla T_{\epsilon} T_{\epsilon} d x+\frac{\sigma}{\epsilon} \int_{\Gamma_{\epsilon}} G_{\epsilon}\left(T_{\epsilon}\right) T_{\epsilon} d s=\int_{\Omega_{\epsilon}^{S}} f T_{\epsilon} d x .
$$


Since $\operatorname{div} V_{\epsilon}=0$ in $\Omega_{\epsilon}^{F}, V_{\epsilon} \cdot n=0$ on $\Gamma_{\epsilon}$ and $T_{\epsilon}=0$ on $\partial \Omega$, we have

$$
\int_{\Omega_{\epsilon}^{F}} V_{\epsilon} \cdot \nabla T_{\epsilon} T_{\epsilon} d x=0
$$

Furthermore, since $G_{\epsilon}$ is a positive operator (see Lemma 2.1)

$$
\int_{\Gamma_{\epsilon}} G_{\epsilon}\left(T_{\epsilon}\right) T_{\epsilon} d s \geq 0
$$

Consequently, by the coercivity of $K_{\epsilon}$, we obtain

$$
\left\|\nabla T_{\epsilon}\right\|_{L^{2}\left(\Omega_{\epsilon}^{S}\right)}^{2}+\epsilon\left\|\nabla T_{\epsilon}\right\|_{L^{2}\left(\Omega_{\epsilon}^{F}\right)}^{2} \leq C\left\|T_{\epsilon}\right\|_{L^{2}\left(\Omega_{\epsilon}^{S}\right)} .
$$

Using Lemma 4.1 we deduce

$$
\left\|\nabla T_{\epsilon}\right\|_{L^{2}\left(\Omega_{\epsilon}^{S}\right)} \leq C .
$$

On the other hand, using Lemma 4.3 and formula (56) yields

$$
\begin{aligned}
\left\|T_{\epsilon}\right\|_{L^{2}(\Omega)}^{2} & \leq C\left[\left\|T_{\epsilon}\right\|_{L^{2}\left(\Omega_{\epsilon}^{S}\right)}^{2}+\epsilon^{2}\left\|\nabla T_{\epsilon}\right\|_{L^{2}(\Omega)}^{2}\right] \\
& \leq C\left[1+\epsilon\left\|\nabla T_{\epsilon}\right\|_{L^{2}\left(\Omega_{\epsilon}^{F}\right)}^{2}\right] \\
& \leq C\left[1+\left\|T_{\epsilon}\right\|_{L^{2}(\Omega)}\right]
\end{aligned}
$$

(since $\epsilon<1$ ) from which we deduce

$$
\left\|T_{\epsilon}\right\|_{L^{2}(\Omega)} \leq C
$$

By (58), and using (56) again, we get

$$
\sqrt{\epsilon}\left\|\nabla T_{\epsilon}\right\|_{L^{2}\left(\Omega_{\epsilon}^{F}\right)} \leq C
$$

Using (58) and (57) and Lemma 4.2 we deduce

$$
\sqrt{\epsilon}\left\|T_{\epsilon}\right\|_{L^{2}\left(\Gamma_{\epsilon}\right)} \leq C .
$$

Combining (57), (58), (59) and (60) we obtain the desired a priori estimate (54).

Lemma 4.1. (see Lemma A.4 in [5]) There exists a constant $C>0$, not depending on $\epsilon$, such that for any function $u \in H^{1}\left(\Omega_{\epsilon}^{S}\right)$ satisfying $u=0$ on $\partial \Omega \cap \partial \Omega_{\epsilon}^{S}$

$$
\|u\|_{L^{2}\left(\Omega_{\epsilon}^{S}\right)} \leq C\|\nabla u\|_{L^{2}\left(\Omega_{\epsilon}^{S}\right)} .
$$

Lemma 4.2. (see Lemma 4.2.4 in [3]) There exists a constant $C>0$, not depending on $\epsilon$, such that

$$
\sqrt{\epsilon}\|u\|_{L^{2}\left(\Gamma_{\epsilon}\right)} \leq C\|u\|_{H^{1}\left(\Omega_{\epsilon}^{S}\right)} \quad \forall u \in H^{1}\left(\Omega_{\epsilon}^{S}\right) .
$$

Lemma 4.3. There exists a constant $C>0$, not depending on $\epsilon$, such that

$$
\|u\|_{L^{2}\left(\Omega_{\epsilon}^{F}\right)} \leq C\left[\|u\|_{L^{2}\left(\Omega_{\epsilon}^{S}\right)}+\epsilon\|\nabla u\|_{L^{2}\left(\Omega_{\epsilon}\right)}\right] \quad \forall u \in H^{1}(\Omega) .
$$


Proof The proof of Lemma 4.3 is similar to those of the previous lemmas so we content ourselves in briefly sketching it. We denote by $Y=\Lambda \times(0,1)$ a $3 \mathrm{D}$ unit cell and similarly $Y^{F, S}=\Lambda^{F, S} \times(0,1)$. By an obvious rescaling and summation argument, it is enough to prove that there exists a constant $C$, not depending on $\epsilon$, such that

$$
\|u\|_{L^{2}\left(Y^{F}\right)}^{2} \leq C\left[\|u\|_{L^{2}\left(Y^{S}\right)}^{2}+\|\nabla u\|_{L^{2}(Y)}^{2}\right] \quad \forall u \in H^{1}(Y) .
$$

We prove (63) by contradiction. Indeed, we suppose that it does not hold true, namely there exists a sequence $\phi_{n} \in H^{1}(Y)$, for $n \geq 1$, such that

$$
\left\|\phi_{n}\right\|_{L^{2}\left(Y^{F}\right)}=1 \text { and }\left\|\phi_{n}\right\|_{L^{2}\left(Y^{S}\right)}^{2}+\left\|\nabla \phi_{n}\right\|_{L^{2}(Y)}^{2}<\frac{1}{n} .
$$

Up to a subsequence, $\phi_{n}$ converges weakly in $H^{1}(Y)$ to a limit $\phi$, and by Rellich theorem this convergence is strong in $L^{2}(Y)$. However, (64) tells us that $\nabla \phi_{n}$ converges strongly to 0 in $L^{2}(Y)$. Therefore, $\nabla \phi=0$ and $\phi$ is constant in $Y$. Once again, (64) implies that this constant is zero in $Y^{S}$ but this is a contradiction with the fact that $\|\phi\|_{L^{2}\left(Y^{F}\right)}=\lim _{n}\left\|\phi_{n}\right\|_{L^{2}\left(Y^{F}\right)}=1$.

\subsection{Two scale convergence}

In this section we first recall the notion of two-scale convergence [1], [30]. Here, since there is no periodicity in the third space direction, we slightly modify the definition of two-scale convergence (these changes do not affect the proofs in any essential way). Second, we prove a rigorous homogenization result, using the two-scale convergence method, to confirm the result obtained in the previous section.

Definition 4.1. A bounded sequence $u_{\epsilon}$ in $L^{2}(\Omega)$ is said to two-scale converge to a function $u_{0}\left(x, y^{\prime}\right) \in$ $L^{2}(\Omega \times \Lambda)$ if there exists a subsequence still denoted by $u_{\epsilon}$ such that

$$
\lim _{\epsilon \rightarrow 0} \int_{\Omega} u_{\epsilon}(x) \psi\left(x, \frac{x^{\prime}}{\epsilon}\right) d x=\frac{1}{|\Lambda|} \int_{\Omega} \int_{\Lambda} u_{0}\left(x, y^{\prime}\right) \psi\left(x, y^{\prime}\right) d x d y^{\prime}
$$

for any $\Lambda$-periodic test function $\psi\left(x, y^{\prime}\right) \in L^{2}\left(\Omega ; C_{\#}(\Lambda)\right)$.

This notion of "two-scale convergence" makes sense because of the next compactness theorem [1], [30].

Theorem 4.1. From each bounded sequence $u_{\epsilon}$ in $L^{2}(\Omega)$, we can extract a subsequence and there exists a limit $u_{0}\left(x, y^{\prime}\right) \in L^{2}(\Omega \times \Lambda)$ such that this subsequence two-scale converges to $u_{0}$.

The extension of Theorem 4.1 to bounded sequences in $H^{1}(\Omega)$ is given next.

Proposition 4.2. From each bounded sequence $u_{\epsilon}$ in $H^{1}(\Omega)$, we can extract a subsequence and there exist two limits $u_{0} \in H^{1}(\Omega)$ and $u_{1}\left(x, y^{\prime}\right) \in L^{2}\left(\Omega ; H_{\#}^{1}(\Lambda)\right)$ such that, for this subsequence, $u_{\epsilon}$ converges weakly to $u_{0}$ in $H^{1}(\Omega)$ and $\nabla u_{\epsilon}$ two-scale converges to $\nabla_{x} u_{0}(x)+\nabla_{y^{\prime}} u_{1}\left(x, y^{\prime}\right)$.

Two-scale convergence can be extended to sequences defined on periodic surfaces [2], [29].

Proposition 4.3. For any sequence $u_{\epsilon}$ in $L^{2}\left(\Gamma_{\epsilon}\right)$ such that

$$
\epsilon \int_{\Gamma_{\epsilon}}\left|u_{\epsilon}\right|^{2} d x \leq C
$$

there exist a subsequence, still denoted $u_{\epsilon}$, and a limit function $u_{0}\left(x, y^{\prime}\right) \in L^{2}\left(\Omega ; L_{\#}^{2}(\gamma)\right)$ such that $u_{\epsilon}$ two-scale converges to $u_{0}$ in the sense

$$
\lim _{\epsilon \rightarrow 0} \epsilon \int_{\Gamma_{\epsilon}} u_{\epsilon}(x) \psi\left(x, \frac{x^{\prime}}{\epsilon}\right) d x=\frac{1}{|\Lambda|} \int_{\Omega} \int_{\gamma} u_{0}\left(x, y^{\prime}\right) \psi\left(x, y^{\prime}\right) d x d y^{\prime}
$$

for any $\Lambda$-periodic test function $\psi\left(x, y^{\prime}\right) \in L^{2}\left(\Omega ; C_{\#}(\gamma)\right)$. 
Remark 4.1. If $u_{\epsilon}$ is a bounded sequence in $H^{1}\left(\Omega_{\epsilon}\right)$, then the uniform bound (66) holds true. It is then easy to check that the two different two-scale limits $u_{0}$ given by Propositions 4.2 and 4.3 coincide [2].

Our main results in this section is the following.

Theorem 4.2. Let $T_{\epsilon}$ be the sequence of solutions of (8). Let $T_{0}(x)$ be the solution of the homogenized problem (25) and $T_{1}\left(x, y^{\prime}\right)$ be the first corrector defined by (28). Then $T_{\epsilon}$ two-scale converges to $T_{0}$ and $\chi_{\epsilon}^{S} \nabla T_{\epsilon}$ two-scale converges to $\chi^{S}\left(y^{\prime}\right)\left(\nabla_{x} T_{0}(x)+\nabla_{y^{\prime}} T_{1}\left(x, y^{\prime}\right)\right)$ where $\chi_{\epsilon}^{S}(x)=\chi^{S}\left(x^{\prime} / \epsilon\right)$ is the characteristic function of $\Omega_{\epsilon}^{S}$ and $\chi^{S}\left(y^{\prime}\right)$ that of $\Lambda^{S}$.

Proof The a priori estimate (54) implies that, up to a subsequence, $T_{\epsilon}$ two-scale converges to a function $T_{0} \in H_{0}^{1}(\Omega)$ and $\chi_{\epsilon}^{S} \nabla T_{\epsilon}$ two-scale converges to $\chi^{S}\left(y^{\prime}\right)\left(\nabla_{x} T_{0}(x)+\nabla_{y} T_{1}\left(x, y^{\prime}\right)\right)$ where $T_{1} \in$ $L^{2}\left(\Omega ; H_{\#}^{1}(\Lambda)\right)$. Furthermore, $T_{\epsilon}$ two-scale converges to $T_{0}$ on the periodic surface $\Gamma_{\epsilon}$, in the sense of Proposition 4.3.

Although we use the same notations, we still have to show that $T_{0}$ is a solution of the homogenized problem (25) and that $T_{1}$ is the first corrector defined by (28). Convergence of the entire sequence (and not merely of an extracted subsequence) will follow from the uniqueness of the solution of (25).

In a first step, we compute the corrector $T_{1}$ in terms of $\nabla_{x} T_{0}$ by choosing the test function $\phi_{\epsilon}(x)=\epsilon \phi_{1}\left(x, \frac{x^{\prime}}{\epsilon}\right)$, where $\phi_{1}\left(x, y^{\prime}\right)$ is any smooth function, compactly supported in $x$ and $\Lambda$-periodic in $y^{\prime}$, in the variational formulation (29) which becomes (using the self-adjoint character of $G_{\epsilon}$ )

$$
\int_{\Omega_{\epsilon}^{S}} K_{\epsilon}^{S} \nabla T_{\epsilon} \cdot \nabla_{y^{\prime}} \phi_{1}+\sigma \int_{\Gamma_{\epsilon}} T_{\epsilon} G_{\epsilon}\left(\phi_{1}\right)=o(1)
$$

where, thanks to the a priori estimate (54),o(1) is a small remainder term going to 0 with $\epsilon$. By virtue of a lower order truncation of formula (15) in Lemma 2.1, the radiative operator can be approximated as

$$
G_{\epsilon}\left(\phi_{1}\right)=\left(I d-\zeta_{\epsilon}\right)\left(\phi_{1}\right)=\left(I d-\zeta_{\epsilon}^{2 D}\right)\left(\phi_{1}\right)+\mathcal{O}\left(\epsilon^{2}\right) .
$$

Then, to pass to the two-scale limit in the radiative term, we rely on Lemma 4.4 below which gives us a smooth periodic vector-valued function $\theta\left(x, y^{\prime}\right)$ such that

$$
\begin{cases}-\operatorname{div}_{y^{\prime}} \theta\left(x, y^{\prime}\right)=0 & \text { in } \Lambda^{S}, \\ \theta\left(x, y^{\prime}\right) \cdot n=\left(I d-\zeta^{2 D}\right) \phi_{1}\left(x, y^{\prime}\right) & \text { on } \gamma \\ y^{\prime} \rightarrow \theta\left(x, y^{\prime}\right) & \text { is } \Lambda \text {-periodic. }\end{cases}
$$

Furthermore, $\theta\left(x, y^{\prime}\right)$ has the same compact support than $\phi_{1}\left(x, y^{\prime}\right)$ with respect to $x \in \Omega$. However, since $\zeta_{\epsilon}^{2 D}$ is an integral operator, we usually have a difference between the two terms below

$$
\zeta_{\epsilon}^{2 D}\left(\phi_{1}\left(x, \frac{x^{\prime}}{\epsilon}\right)\right) \neq\left(\zeta^{2 D}\left(\phi_{1}\left(x, y^{\prime}\right)\right)\right)\left(y^{\prime}=\frac{x^{\prime}}{\epsilon}\right) .
$$

Therefore, we need to use a Taylor expansion of $\phi_{1}$

$$
\phi_{1}\left(x, \frac{x^{\prime}}{\epsilon}\right)=\phi_{1}\left(x_{0, i}, \frac{x^{\prime}}{\epsilon}\right)+\left(x^{\prime}-x_{0, i}^{\prime}\right) \cdot \nabla_{x^{\prime}} \phi_{1}\left(x_{0, i}, \frac{x^{\prime}}{\epsilon}\right)+\mathcal{O}\left(\epsilon^{2}\right),
$$

where $x_{0, i}=\left(x_{0, i}^{\prime}, x_{3}\right)$ and $x_{0, i}^{\prime}$ is the center of mass of each boundary $\gamma_{\epsilon, i}$, defined by (1). Then, the following equality holds true

$$
\zeta_{\epsilon}^{2 D}\left(\phi_{1}\left(x, \frac{x^{\prime}}{\epsilon}\right)\right)=\left(\zeta^{2 D}\left(\phi_{1}\left(x_{0, i}, y^{\prime}\right)\right)\right)\left(y^{\prime}=\frac{x^{\prime}}{\epsilon}\right)+\epsilon\left(\zeta^{2 D}\left(y^{\prime} \cdot \nabla_{x^{\prime}} \phi_{1}\left(x_{0, i}, y^{\prime}\right)\right)\right)\left(y^{\prime}=\frac{x^{\prime}}{\epsilon}\right)+\mathcal{O}\left(\epsilon^{2}\right)
$$

Then, we can rewrite the radiative term in (68) as

$$
\sigma \int_{\Gamma_{\epsilon}} T_{\epsilon} G_{\epsilon}\left(\phi_{1}\right)=\sigma \int_{\Gamma_{\epsilon}} T_{\epsilon} \theta\left(x_{0, i}, \frac{x^{\prime}}{\epsilon}\right) \cdot n+\sigma \epsilon \int_{\Gamma_{\epsilon}} T_{\epsilon}\left(\left(I d-\zeta^{2 D}\right)\left(y^{\prime} \cdot \nabla_{x^{\prime}} \phi_{1}\left(x_{0, i}, y^{\prime}\right)\right)\right)\left(y^{\prime}=\frac{x^{\prime}}{\epsilon}\right)+\mathcal{O}(\epsilon) .
$$


We can pass to the two-scale limit in the second term in the right hand side of (69) by applying Proposition 4.3 (replacing $y^{\prime} \cdot \nabla_{x^{\prime}} \phi_{1}\left(x_{0, i}, y^{\prime}\right)$ by the suitable two-scale test function $y^{\prime} \cdot \nabla_{x^{\prime}} \phi_{1}\left(x, y^{\prime}\right)$ up to another $\mathcal{O}(\epsilon)$ error). For the first term, we use a similar Taylor expansion for $\theta$

$$
\begin{aligned}
\sigma \int_{\Gamma_{\epsilon}} T_{\epsilon} \theta\left(x_{0, i}, \frac{x^{\prime}}{\epsilon}\right) \cdot n & =\sigma \int_{\Gamma_{\epsilon}} T_{\epsilon} \theta\left(x, \frac{x^{\prime}}{\epsilon}\right) \cdot n-\epsilon \sigma \int_{\Gamma_{\epsilon}} T_{\epsilon}\left(y^{\prime} \cdot \nabla_{x^{\prime}} \theta\left(x, y^{\prime}\right)\right)\left(y^{\prime}=\frac{x^{\prime}}{\epsilon}\right) \cdot n+\mathcal{O}(\epsilon) \\
& =\sigma \int_{\Omega_{\epsilon}^{S}} \operatorname{div}\left(T_{\epsilon} \theta\left(x, \frac{x^{\prime}}{\epsilon}\right)-\epsilon \sigma \int_{\Gamma_{\epsilon}} T_{\epsilon}\left(y^{\prime} \cdot \nabla_{x^{\prime}} \theta\left(x, y^{\prime}\right)\right)\left(y^{\prime}=\frac{x^{\prime}}{\epsilon}\right) \cdot n+\mathcal{O}(\epsilon \nmid 70)\right.
\end{aligned}
$$

For the second integral in (70) we can pass to the two-scale limit by another application of Proposition 4.3. Concerning the first integral, we develop

$$
\operatorname{div}\left(T_{\epsilon}(x) \theta\left(x, \frac{x^{\prime}}{\epsilon}\right)\right)=\nabla T_{\epsilon}(x) \cdot \theta\left(x, \frac{x^{\prime}}{\epsilon}\right)+T_{\epsilon}(x)\left(\operatorname{div}_{x} \theta\right)\left(x, \frac{x^{\prime}}{\epsilon}\right),
$$

and we can pass to the two-scale limit, thanks to Proposition 4.2. All in all, after some integration by parts, and recalling that $\operatorname{ker}\left(I d-\zeta_{\epsilon}^{2 D}\right)=\mathbb{R}$, we get

$$
\begin{aligned}
\lim _{\epsilon \rightarrow 0} \sigma \int_{\Gamma_{\epsilon}} T_{\epsilon} G_{\epsilon}\left(\phi_{1}\right) & =\frac{\sigma}{|\Lambda|} \int_{\Omega} \int_{\Lambda^{S}}\left(\theta\left(x, y^{\prime}\right) \cdot\left(\nabla T_{0}(x)+\nabla_{y^{\prime}} T_{1}\left(x, y^{\prime}\right)\right)+T_{0}(x) \operatorname{div} x \theta\left(x, y^{\prime}\right)\right) d y^{\prime} d x \\
& +\frac{\sigma}{|\Lambda|} \int_{\Omega} \int_{\gamma} T_{0}(x)\left(\left(I d-\zeta^{2 D}\right)\left(y^{\prime} \cdot \nabla_{x^{\prime}} \phi_{1}\left(x, y^{\prime}\right)\right)-y^{\prime} \cdot \nabla_{x^{\prime}} \theta\left(x, y^{\prime}\right)\right) d y^{\prime} d x \\
& =\frac{\sigma}{|\Lambda|} \int_{\Omega} \int_{\gamma} \theta\left(x, y^{\prime}\right) \cdot n\left(T_{1}\left(x, y^{\prime}\right)+y^{\prime} \cdot \nabla_{x^{\prime}} T_{0}(x)\right) d y^{\prime} d x \\
& =\frac{\sigma}{|\Lambda|} \int_{\Omega} \int_{\gamma}\left(I d-\zeta^{2 D}\right)\left(\phi_{1}\left(x, y^{\prime}\right)\right)\left(T_{1}\left(x, y^{\prime}\right)+y^{\prime} \cdot \nabla_{x^{\prime}} T_{0}(x)\right) d y^{\prime} d x
\end{aligned}
$$

Therefore, the two-scale limit of (68) is

$$
\begin{array}{r}
\int_{\Omega} \int_{\Lambda^{S}} K^{S}\left(x, y^{\prime}\right)\left(\nabla_{x} T_{0}(x)+\nabla_{y^{\prime}} T_{1}\left(x, y^{\prime}\right)\right) \cdot \nabla_{y^{\prime}} \phi_{1}\left(x, y^{\prime}\right) d y^{\prime} d x \\
+\sigma \int_{\Omega} \int_{\gamma}\left(I d-\zeta^{2 D}\right) \phi_{1}\left(x, y^{\prime}\right)\left(T_{1}\left(x, y^{\prime}\right)+y^{\prime} \cdot \nabla_{x^{\prime}} T_{0}(x)\right) d y^{\prime} d x=0
\end{array}
$$

which is precisely the variational formulation (45) for $T_{1}$. Therefore, we have proved that

$$
T_{1}\left(x, y^{\prime}\right)=\sum_{k=1}^{3} \frac{\partial T_{0}}{\partial x_{k}}(x) \omega_{k}\left(y^{\prime}\right) \quad \text { in } \Omega \times \Lambda^{S} .
$$

Remark that our convergence proof does not justify formula (28) for $T_{1}\left(x, y^{\prime}\right)$ in the fluid part $\Omega \times \Lambda^{F}$.

Remark 4.2. The first step of our proof (which gives formula (28) for $T_{1}\left(x, y^{\prime}\right)$ ) was missing in the proof of Theorem 4.6 in [3]. Our above argument works also in the simpler 2D setting of [3] and is thus filling this gap.

In a second step, we recover the homogenized problem for $T_{0}$ by choosing another test function $\phi_{\epsilon}(x)$ in the variational formulation (29) given by

$$
\phi_{\epsilon}(x)=\phi_{0}(x)+\epsilon \phi_{1}\left(x, \frac{x^{\prime}}{\epsilon}\right) \quad \text { with } \quad \phi_{1}\left(x, y^{\prime}\right)=\sum_{k=1}^{3} \frac{\partial \phi_{0}}{\partial x_{k}}(x) \omega_{k}\left(y^{\prime}\right)
$$


where $\phi_{0} \in C_{c}^{\infty}(\Omega)$ and $\omega_{j}$ are solutions of the cell problems (27). The variational formulation (29) becomes

$$
\begin{array}{r}
\int_{\Omega_{\epsilon}^{S}} K_{\epsilon}^{S} \nabla T_{\epsilon} \cdot\left(\nabla_{x} \phi_{0}+\nabla_{y^{\prime}} \phi_{1}\right)+\epsilon \int_{\Omega_{\epsilon}^{F}} K_{\epsilon}^{F} \nabla T_{\epsilon} \cdot\left(\nabla \phi_{0}+\nabla_{y^{\prime}} \phi_{1}\right) \\
+\int_{\Omega_{\epsilon}^{F}} V_{\epsilon} \cdot \nabla T_{\epsilon}\left(\phi_{0}+\epsilon \phi_{1}\right)+\frac{\sigma}{\epsilon} \int_{\Gamma_{\epsilon}} G_{\epsilon}\left(T_{\epsilon}\right)\left(\phi_{0}+\epsilon \phi_{1}\right)=\int_{\Omega_{\epsilon}^{S}} f \phi_{0}+o(1)
\end{array}
$$

where $o(1)$ is a small remainder term going to 0 with $\epsilon$. Passing to the two-scale limit in all terms, except the radiative one, is standard (see [1] if necessary). Therefore, we focus only on the radiative term

$$
\frac{\sigma}{\epsilon} \int_{\Gamma_{\epsilon}} G_{\epsilon}\left(T_{\epsilon}\right)\left(\phi_{0}+\epsilon \phi_{1}\right)=\frac{\sigma}{\epsilon} \int_{\Gamma_{\epsilon}} T_{\epsilon} G_{\epsilon}\left(\phi_{0}+\epsilon \phi_{1}\right)
$$

for which we generalize an argument of [3]. We write a Taylor expansion of $\phi_{\epsilon}$, with respect to the macroscopic variable only, around the center of mass $x_{0, i}=\left(x_{0, i}^{\prime}, x_{3}\right)$ of each boundary $\gamma_{\epsilon, i}$ (for conciseness we drop the index $i$ in the sequel)

$$
\begin{array}{r}
\phi_{\epsilon}(x)=\phi_{0}\left(x_{0}\right)+\nabla_{x^{\prime}} \phi_{0}\left(x_{0}\right) \cdot\left(x^{\prime}-x_{0}^{\prime}\right)+\epsilon \phi_{1}\left(x_{0}, \frac{x^{\prime}}{\epsilon}\right)+\epsilon \nabla_{x^{\prime}} \phi_{1}\left(x_{0}, \frac{x^{\prime}}{\epsilon}\right) \cdot\left(x^{\prime}-x_{0}^{\prime}\right) \\
+\frac{1}{2} \nabla_{x^{\prime}} \nabla_{x^{\prime}} \phi_{0}\left(x_{0}\right)\left(x^{\prime}-x_{0}^{\prime}\right) \cdot\left(x^{\prime}-x_{0}^{\prime}\right)+\mathcal{O}\left(\epsilon^{3}\right) .
\end{array}
$$

We go up to second order in this Taylor expansion since, upon dividing by $\epsilon$ as in (72) and summing over all boundaries $\gamma_{\epsilon, i}$, they will have a non-zero limit according to Proposition 4.3. Recall that the solution of the cell problem $\omega_{3}^{S}$ in the $x_{3}$ direction is a constant in $\Lambda^{S}$ (see Remark 3.2): we can choose this constant to be zero so that $\omega_{3}^{S}\left(y^{\prime}\right)=0$ in $\Lambda^{S}$ and thus on the boundary $\gamma$ too. Therefore, in the boundary integral (72) we can write that the test function $\phi_{1}$ is just

$$
\phi_{1}\left(x, y^{\prime}\right)=\sum_{k=1}^{2} \frac{\partial \phi_{0}}{\partial x_{k}}(x) \omega_{k}\left(y^{\prime}\right) \quad \text { on } \gamma,
$$

without any contribution in the $x_{3}$ direction. Thus, the radiation term is given by

$$
\frac{1}{\epsilon} G_{\epsilon}\left(\phi_{0}+\epsilon \phi_{1}\right)(x)=\frac{1}{\epsilon}\left(I d-\zeta_{\epsilon}\right)\left(\phi_{0}+\epsilon \phi_{1}\right)(x)=\epsilon\left(\psi_{0, \epsilon}(x)+\psi_{1, \epsilon}(x)+\psi_{2, \epsilon}(x)+\mathcal{O}(\epsilon)\right)
$$

where

$$
\begin{aligned}
\psi_{0, \epsilon}(x)= & \frac{1}{\epsilon^{2}}\left(I d-\zeta_{\epsilon}\right)\left[\phi_{0}\left(x_{0}\right)\right] \\
\psi_{1, \epsilon}(x)= & \frac{1}{\epsilon} \sum_{k=1}^{2}\left(I d-\zeta_{\epsilon}\right)\left[\left(\omega_{k}\left(\frac{x^{\prime}}{\epsilon}\right)+\frac{x_{k}-x_{0, k}}{\epsilon}\right) \frac{\partial \phi_{0}}{\partial x_{k}}\left(x_{0}\right)\right] \\
\psi_{2, \epsilon}(x)= & \left(I d-\zeta_{\epsilon}\right)\left[\frac{1}{2} \nabla_{x^{\prime}} \nabla_{x^{\prime}} \phi_{0}\left(x_{0}\right) \cdot \frac{\left(x^{\prime}-x_{0}^{\prime}\right)}{\epsilon} \otimes \frac{\left(x^{\prime}-x_{0}^{\prime}\right)}{\epsilon}\right. \\
& \left.+\sum_{k=1}^{2} \nabla_{x^{\prime}} \frac{\partial \phi_{0}}{\partial x_{k}}\left(x_{0}\right) \cdot\left(\frac{\left(x^{\prime}-x_{0}^{\prime}\right)}{\epsilon} \omega_{k}\left(\frac{x^{\prime}}{\epsilon}\right)\right)\right] .
\end{aligned}
$$

In (73), when the integral operator $\zeta_{\epsilon}$ is applied to a function depending on $x_{0}=\left(x_{0}^{\prime}, x_{3}\right)$, it is meant that $x_{0}^{\prime}$ is constant (for all points on $\Gamma_{\epsilon, i}$ while $x_{3}$ is varying. In other words, for a given function $g$, we have

$$
\zeta_{\epsilon}\left[g\left(x_{0}\right)\right](s)=\int_{\Gamma_{\epsilon, i}} F(s, x) g\left(x_{0}^{\prime}, x_{3}\right) d x .
$$


Remark 4.3. At this point, for simplicity we assume that the periodic diffusion coefficients $K^{S, F}$ and the velocity $V$ do not depend on $x$. Otherwise, this would add further terms in (73) corresponding to the $x$ derivatives of the cell solutions $\omega_{k}$. Our arguments would still work but we prefer to simplify the exposition.

The term $\psi_{0, \epsilon}$ is new compared to the $2 \mathrm{D}$ setting in [3] (where it was vanishing). Furthermore, the main additional difficulty with respect to [3] is that we need to approximate the $3 \mathrm{D}$ view factor in $\zeta_{\epsilon}$ by the $2 \mathrm{D}$ view factor which is appearing in the homogenized limit. For this goal we rely on Lemma 2.1. First, by virtue of (15), for any $0<p<3$ we have

$$
\psi_{0, \epsilon}(x)=-\frac{1}{2 \epsilon^{2}} \frac{\partial^{2} \phi_{0}}{\partial x_{3}^{2}}\left(x_{0}\right) \int_{\gamma_{\epsilon, i}} F^{2 D}\left(s^{\prime}, x^{\prime}\right)\left|x^{\prime}-s^{\prime}\right|^{2} d s^{\prime}+\mathcal{O}\left(\epsilon^{p-2}\right) .
$$

Second, by a lower order truncation of (15), and since $\omega_{k}$ does not depend on $x_{3}$,

$$
\psi_{1, \epsilon}(x)=\frac{1}{\epsilon} \sum_{k=1}^{2} \frac{\partial \phi_{0}}{\partial x_{k}}\left(x_{0}\right)\left(I d-\zeta_{\epsilon}^{2 D}\right)\left(\omega_{k}\left(\frac{x^{\prime}}{\epsilon}\right)+\frac{x_{k}-x_{0, k}}{\epsilon}\right)+\mathcal{O}(\epsilon)
$$

and

$$
\begin{aligned}
\psi_{2, \epsilon}(x)= & \frac{1}{2} \nabla_{x^{\prime}} \nabla_{x^{\prime}} \phi_{0}\left(x_{0}\right) \cdot\left(I d-\zeta_{\epsilon}^{2 D}\right)\left(\frac{\left(x^{\prime}-x_{0}^{\prime}\right)}{\epsilon} \otimes \frac{\left(x^{\prime}-x_{0}^{\prime}\right)}{\epsilon}\right) \\
& +\sum_{k=1}^{2} \nabla_{x^{\prime}} \frac{\partial \phi_{0}}{\partial x_{k}}\left(x_{0}\right) \cdot\left(I d-\zeta_{\epsilon}^{2 D}\right)\left(\frac{\left(x^{\prime}-x_{0}^{\prime}\right)}{\epsilon} \omega_{k}\left(\frac{x^{\prime}}{\epsilon}\right)\right)+\mathcal{O}\left(\epsilon^{2}\right) .
\end{aligned}
$$

In order to recover continuous functions, we use the following Taylor expansions

$$
\begin{aligned}
\frac{\partial \phi_{0}}{\partial x_{k}}\left(x_{0}\right) & =\frac{\partial \phi_{0}}{\partial x_{k}}(x)-\nabla_{x^{\prime}} \frac{\partial \phi_{0}}{\partial x_{k}}(x) \cdot\left(x^{\prime}-x_{0}^{\prime}\right)+\mathcal{O}\left(\epsilon^{2}\right), \\
\frac{\partial^{2} \phi_{0}}{\partial x_{3}^{2}}\left(x_{0}\right) & =\frac{\partial^{2} \phi_{0}}{\partial x_{3}^{2}}(x)+\mathcal{O}(\epsilon) .
\end{aligned}
$$

We get

$$
\begin{aligned}
\psi_{0, \epsilon}(x)= & -\frac{1}{2} \frac{\partial^{2} \phi_{0}}{\partial x_{3}^{2}}(x) \int_{\gamma_{\epsilon, i}} F^{2 D}\left(s^{\prime}, x^{\prime}\right) \frac{\left|x^{\prime}-s^{\prime}\right|^{2}}{\epsilon^{2}} d s^{\prime}+\mathcal{O}\left(\epsilon^{p-2}\right), \\
\psi_{1, \epsilon}(x)= & \frac{1}{\epsilon} \sum_{k=1}^{2}\left(\frac{\partial \phi_{0}}{\partial x_{k}}(x)-\nabla_{x^{\prime}} \frac{\partial \phi_{0}}{\partial x_{k}}(x) \cdot\left(x^{\prime}-x_{0}^{\prime}\right)\right)\left(I d-\zeta_{\epsilon}^{2 D}\right)\left(\omega_{k}\left(\frac{x^{\prime}}{\epsilon}\right)+\frac{x_{k}-x_{0, k}}{\epsilon}\right)+\mathcal{O}(\epsilon), \\
\psi_{2, \epsilon}(x)= & \frac{1}{2} \nabla_{x^{\prime}} \nabla_{x^{\prime}} \phi_{0}(x) \cdot\left(I d-\zeta_{\epsilon}^{2 D}\right)\left(\frac{\left(x^{\prime}-x_{0}^{\prime}\right)}{\epsilon} \otimes \frac{\left(x^{\prime}-x_{0}^{\prime}\right)}{\epsilon}\right) \\
& +\sum_{k=1}^{2} \nabla_{x^{\prime}} \frac{\partial \phi_{0}}{\partial x_{k}}(x) \cdot\left(I d-\zeta_{\epsilon}^{2 D}\right)\left(\frac{\left(x^{\prime}-x_{0}^{\prime}\right)}{\epsilon} \omega_{k}\left(\frac{x^{\prime}}{\epsilon}\right)\right)+\mathcal{O}(\epsilon) .
\end{aligned}
$$

The leading term of $\psi_{0, \epsilon}(x)$ is precisely an oscillating test function for two-scale convergence

$$
\psi_{0, \epsilon}(x)=\psi_{0}\left(x, \frac{x^{\prime}}{\epsilon}\right)+\mathcal{O}\left(\epsilon^{p-2}\right) \quad \text { with } \psi_{0}\left(x, y^{\prime}\right)=-\frac{1}{2} \frac{\partial^{2} \phi_{0}}{\partial x_{3}^{2}}(x) \int_{\gamma} F^{2 D}\left(z^{\prime}, y^{\prime}\right)\left|y^{\prime}-z^{\prime}\right|^{2} d z^{\prime} .
$$


The same is true for $\psi_{2, \epsilon}(x)$ which is also an oscillating test function for two-scale convergence

$$
\psi_{2, \epsilon}(x)=\psi_{2}\left(x, \frac{x^{\prime}}{\epsilon}\right)+\mathcal{O}(\epsilon)
$$

with

$$
\psi_{2}\left(x, y^{\prime}\right)=\frac{1}{2} \nabla_{x^{\prime}} \nabla_{x^{\prime}} \phi_{0}(x) \cdot\left(I d-\zeta^{2 D}\right)\left(y^{\prime} \otimes y^{\prime}\right)+\sum_{k=1}^{2} \nabla_{x^{\prime}} \frac{\partial \phi_{0}}{\partial x_{k}}(x) \cdot\left(I d-\zeta^{2 D}\right)\left(y^{\prime} \omega_{k}\left(y^{\prime}\right)\right) .
$$

Rewriting the radiative term (72) as

$$
\frac{\sigma}{\epsilon} \int_{\Gamma_{\epsilon}} T_{\epsilon} G_{\epsilon}\left(\phi_{0}+\epsilon \phi_{1}\right)=\sigma \epsilon \int_{\Gamma_{\epsilon}} T_{\epsilon}\left(\psi_{0, \epsilon}(x)+\psi_{1, \epsilon}(x)+\psi_{2, \epsilon}(x)+\mathcal{O}(\epsilon)\right),
$$

we can pass to the two-scale limit in the first and third term in the right hand side of (75) by application of Proposition 4.3. We obtain

$$
\lim _{\epsilon \rightarrow 0} \sigma \epsilon \sum_{i=1}^{N(\epsilon)} \int_{\Gamma_{\epsilon, i}} \psi_{0, \epsilon} T_{\epsilon}=\frac{-\sigma}{2|\Lambda|} \int_{\Omega} T_{0}(x) \frac{\partial^{2} \phi_{0}}{\partial x_{3}^{2}}(x) \int_{\gamma} \int_{\gamma} F^{2 D}\left(y^{\prime}, z^{\prime}\right)\left|y^{\prime}-z^{\prime}\right|^{2} d y^{\prime} d z^{\prime} d x .
$$

and

$$
\lim _{\epsilon \rightarrow 0} \sigma \epsilon \sum_{i=1}^{N(\epsilon)} \int_{\Gamma_{\epsilon, i}} \psi_{2, \epsilon} T_{\epsilon}=\frac{\sigma}{|\Lambda|} \int_{\Omega} T_{0}(x) \int_{\gamma} \psi_{2}\left(x, y^{\prime}\right) d y^{\prime} d x=0
$$

because, by the second property of Lemma 2.1, we have $\int_{\gamma} \psi_{2}\left(x, y^{\prime}\right) d y^{\prime}=0$.

It remains to pass to the limit in the second term of (75) involving $\psi_{1, \epsilon}$. Following [3] we use the classical trick of $H$-convergence [28] which amounts to make a comparison with the variational formulation of the cell problems (46) with the test function $T_{\epsilon} \frac{\partial \phi_{0}}{\partial x_{k}}$ (recall that $\phi_{0}$ has compact support). From (46), after rescaling and integration with respect to $x_{3}$, we obtain for $k=1,2$

$$
\sigma \int_{\Gamma_{\epsilon}}\left(I d-\zeta_{\epsilon}^{2 D}\right)\left(\omega_{k}\left(\frac{x^{\prime}}{\epsilon}\right)+\frac{x_{k}-x_{0, k}}{\epsilon}\right)\left(T_{\epsilon} \frac{\partial \phi_{0}}{\partial x_{k}}\right)=-\int_{\Omega_{\epsilon}^{S}} K_{\epsilon}^{S}\left(\nabla_{y^{\prime}} \omega_{k}\left(\frac{x^{\prime}}{\epsilon}\right)+e_{k}\right) \cdot \nabla_{x^{\prime}}\left(T_{\epsilon} \frac{\partial \phi_{0}}{\partial x_{k}}\right)
$$

which implies

$$
\begin{aligned}
\epsilon \sigma \int_{\Gamma_{\epsilon}} \psi_{1, \epsilon}(x) T_{\epsilon}(x) & =-\sum_{k=1}^{2} \int_{\Omega_{\epsilon}^{S}} K_{\epsilon}^{S}\left(\nabla_{y^{\prime}} \omega_{k}\left(\frac{x^{\prime}}{\epsilon}\right)+e_{k}\right) \cdot \nabla_{x^{\prime}}\left(T_{\epsilon} \frac{\partial \phi_{0}}{\partial x_{k}}\right) \\
& -\sigma \epsilon \sum_{k=1}^{2} \int_{\Gamma_{\epsilon}}\left(I d-\zeta_{\epsilon}^{2 D}\right)\left(\omega_{k}\left(\frac{x^{\prime}}{\epsilon}\right)+\frac{x_{k}-x_{0, k}}{\epsilon}\right)\left(\nabla_{x^{\prime}} \frac{\partial \phi}{\partial x_{k}}(x) \cdot \frac{x^{\prime}-x_{0}^{\prime}}{\epsilon}\right) T_{\epsilon} .
\end{aligned}
$$

It is now possible to pass to the two-scale limit in the right hand side of (78) and, summing up those 
limits, we deduce

$$
\begin{aligned}
& \lim _{\epsilon \rightarrow 0} \frac{\sigma}{\epsilon} \int_{\Gamma_{\epsilon}} G_{\epsilon}\left(T_{\epsilon}\right)\left(\phi_{0}+\epsilon \phi_{1}\right) \\
= & \frac{-\sigma}{2|\Lambda|} \int_{\Omega} T_{0}(x) \frac{\partial^{2} \phi_{0}}{\partial x_{3}^{2}}(x) \int_{\gamma} \int_{\gamma} F^{2 D}\left(y^{\prime}, z^{\prime}\right)\left|y^{\prime}-z^{\prime}\right|^{2} d y^{\prime} d z^{\prime} d x \\
- & \frac{1}{|\Lambda|} \sum_{k=1}^{2} \int_{\Omega} \int_{\Lambda^{S}} K^{S}\left(e_{k}+\nabla_{y^{\prime}} \omega_{k}\left(y^{\prime}\right)\right) \cdot\left(\nabla_{x^{\prime}}\left(T_{0} \frac{\partial \phi_{0}}{\partial x_{k}}\right)(x)+\frac{\partial \phi_{0}}{\partial x_{k}}(x) \nabla_{y^{\prime}} T_{1}\left(x, y^{\prime}\right)\right) d y^{\prime} d x \\
- & \frac{\sigma}{|\Lambda|} \sum_{k=1}^{2} \int_{\Omega} \int_{\gamma}\left(I d-\zeta^{2 D}\right)\left(\omega_{k}+y_{k}\right) y^{\prime} \cdot \nabla_{x^{\prime}} \frac{\partial \phi_{0}}{\partial x_{k}} T_{0} d y^{\prime} d x \\
= & \frac{-\sigma}{2|\Lambda|} \int_{\Omega} T_{0}(x) \frac{\partial^{2} \phi_{0}}{\partial x_{3}^{2}}(x) \int_{\gamma} \int_{\gamma} F^{2 D}\left(y^{\prime}, z^{\prime}\right)\left|y^{\prime}-z^{\prime}\right|^{2} d y^{\prime} d z^{\prime} d x \\
- & \frac{1}{|\Lambda|} \int_{\Omega} \int_{\Lambda^{S}} K^{S}\left(\nabla_{x^{\prime}} T_{0}+\nabla_{y^{\prime}} T_{1}\right) \cdot\left(\nabla_{x^{\prime}} \phi_{0}+\nabla_{y^{\prime}} \phi_{1}\right) d y^{\prime} d x-\int_{\Omega} K^{*} T_{0} \nabla_{x^{\prime}} \nabla_{x^{\prime}} \phi_{0} d x . \\
= & \int_{\Omega} K^{*}(x) \nabla_{x} T_{0}(x) \cdot \nabla_{x} \phi_{0}(x) d x-\frac{1}{|\Lambda|} \int_{\Omega} \int_{\Lambda^{S}} K^{S}\left(\nabla_{x} T_{0}+\nabla_{y^{\prime}} T_{1}\right) \cdot\left(\nabla_{x} \phi_{0}+\nabla_{y^{\prime}} \phi_{1}\right) d y^{\prime} d x
\end{aligned}
$$

The two last equalities in (79) hold true thanks to the following equivalent formula for the homogenized conductivity

$$
K_{j, k}^{*}(x)=\frac{1}{|\Lambda|}\left[\int_{\Lambda^{S}} K^{S}\left(x, y^{\prime}\right)\left(e_{j}+\nabla_{y^{\prime}} \omega_{j}^{S}\left(x, y^{\prime}\right)\right) \cdot e_{k} d y^{\prime}+\sigma \int_{\gamma}\left(I d-\zeta^{2 D}\right)\left(\omega_{j}^{S}\left(x, y^{\prime}\right)+y_{j}\right) y_{k} d y^{\prime}\right]
$$

which is obtained by a combination of (26) and of the variational formulation of the cell problems. The two-scale limits of the other terms in the variational formulation (71) are easily obtained

$$
\begin{aligned}
\lim _{\epsilon \rightarrow 0} \int_{\Omega_{\epsilon}^{S}} K_{\epsilon}^{S} \nabla T_{\epsilon} \cdot\left(\nabla_{x} \phi_{0}+\nabla_{y^{\prime}} \phi_{1}\right) d x= & \frac{1}{|\Lambda|} \int_{\Omega} \int_{\Lambda^{S}} K^{S}\left(\nabla_{x} T_{0}+\nabla_{y^{\prime}} T_{1}\right) \cdot\left(\nabla_{x} \phi_{0}+\nabla_{y^{\prime}} \phi_{1}\right) d y^{\prime} d x \\
\lim _{\epsilon \rightarrow 0} \epsilon \int_{\Omega_{\epsilon}^{F}} K_{\epsilon}^{F} \nabla T_{\epsilon} \cdot\left(\nabla_{x} \phi_{0}+\nabla_{y^{\prime}} \phi_{1}\right) d x & =0 \\
\lim _{\epsilon \rightarrow 0} \int_{\Omega_{\epsilon}^{F}} V_{\epsilon} \cdot \nabla T_{\epsilon}\left(\phi_{0}+\epsilon \phi_{1}\right) d x & =-\lim _{\epsilon \rightarrow 0} \int_{\Omega_{\epsilon}^{F}} T_{\epsilon} V_{\epsilon} \cdot\left(\nabla_{x} \phi_{0}+\nabla_{y^{\prime}} \phi_{1}\right) d x \\
& =-\frac{1}{|\Lambda|} \int_{\Omega} \int_{\Lambda^{F}} T_{0} V\left(y^{\prime}, x\right) \cdot\left(\nabla_{x} \phi_{0}+\nabla_{y^{\prime}} \phi_{1}\right) d y^{\prime} d x \\
& =\frac{1}{|\Lambda|} \int_{\Omega} \int_{\Lambda^{F}} V\left(y^{\prime}, x\right) \cdot \nabla_{x} T_{0} \phi_{0} d y^{\prime} d x,
\end{aligned}
$$

by integration by parts and our assumptions on the velocity $V$. Summing up all those terms we deduce that the limit of the variational formulation (71) is, up to some integration by parts, the variational formulation (47) of the homogenized problem.

Remark 4.4. In the course of the proof of Theorem 4.2 we use in an essential way the fact that the boundary condition on $\partial \Omega$ is of Dirichlet type. For example, it was crucial that the test function had a compact support (at least in $x_{3}$ ) to apply Lemma 2.1 on the 3D to $2 D$ reduction of the view factor. We do not know if the convergence proof can be extended to the case of Neumann boundary conditions.

We now state and prove a technical result which was required in the previous proof. 
Lemma 4.4. Let $\phi_{1}\left(x, y^{\prime}\right)$ be a smooth function, compactly supported in $x \in \Omega$ and $\Lambda$-periodic in $y^{\prime}$. There exists at least one smooth vector-valued function $\theta\left(x, y^{\prime}\right)$ (with values in $\mathbb{R}^{2}$ ) such that

$$
\left\{\begin{array}{rr}
-\operatorname{div}_{y^{\prime}} \theta\left(x, y^{\prime}\right)=0 & \text { in } \Lambda^{S}, \\
\theta\left(x, y^{\prime}\right) \cdot n=\left(I d-\zeta^{2 D}\right) \phi_{1}\left(x, y^{\prime}\right) & \text { on } \gamma, \\
y^{\prime} \rightarrow \theta\left(x, y^{\prime}\right) & \text { is } \Lambda \text {-periodic. }
\end{array}\right.
$$

Proof It is enough to look for a solution under the form $\theta\left(x, y^{\prime}\right)=\nabla_{y^{\prime}} \eta\left(x, y^{\prime}\right)$. To solve the 2D elliptic equation for $\eta$ (in $H_{\#}^{1}\left(\Lambda^{S}\right) / \mathbb{R}$ ), corresponding to (80), we just have to check the compatibility condition of the data (or Fredholm alternative). By virtue of the second property of Lemma 2.1 we can check that, indeed,

$$
\int_{\gamma}\left(I d-\zeta^{2 D}\right) \phi_{1}\left(x, y^{\prime}\right) d y^{\prime}=\int_{\gamma} \phi_{1}\left(x, y^{\prime}\right) d y^{\prime}-\int_{\gamma} \int_{\gamma} F^{2 D}\left(y^{\prime}, s^{\prime}\right) \phi_{1}\left(x, y^{\prime}\right) d y^{\prime} d s^{\prime}=0 .
$$

There is no uniqueness of the solution $\theta\left(x, y^{\prime}\right)$ to which we can add any solenoidal field with zero normal trace.

\subsection{Strong convergence}

Our main result, Theorem 4.2, gives only a weak convergence (or two-scale convergence) of the sequences $T_{\epsilon}$ and $\nabla T_{\epsilon}$. The goal of our next result is to improve this weak convergence into a strong one. As usual in homogenization theory it requires some additional smoothness assumptions. More precisely, we need $T_{1}\left(x, x^{\prime} / \epsilon\right)$ to belong to the space $H^{1}(\Omega)$ (but not to be uniformly bounded). This is true, of course, if $T_{1}\left(x, y^{\prime}\right)$ is a smooth function of $\left(x, y^{\prime}\right)$. In view of formula (28) for $T_{1}$, it is enough that either the homogenized solution $T_{0}(x)$ or the cell solutions $\omega_{k}\left(y^{\prime}\right)$ be smooth. To establish our strong convergence result we rely on the usual energy convergence trick (as described in [1] in the context of two-scale convergence) which is inspired from the notion of $\Gamma$-convergence [17].

Theorem 4.3. Assuming that $T_{1}\left(x, y^{\prime}\right)$ is smooth enough and denoting by $\chi_{\epsilon}^{S}$ the characteristic function of the solid part $\Omega_{\epsilon}^{S}$, the sequence $\left(\nabla T_{\epsilon}(x)-\nabla T_{0}(x)-\nabla_{y^{\prime}} T_{1}\left(x, \frac{x^{\prime}}{\epsilon}\right)\right) \chi_{\epsilon}^{S}$ converges strongly to zero in $L^{2}(\Omega)^{d}$ and the sequence $\left(T_{\epsilon}(x)-T_{0}(x)\right)$ converges strongly to zero in $L^{2}(\Omega)$.

Proof We develop the "energy" of the difference $T_{\epsilon}(x)-T_{0}(x)-\epsilon T_{1}\left(x, \frac{x^{\prime}}{\epsilon}\right)$ and we get, using the energy equality (55)

$$
\begin{aligned}
& \int_{\Omega_{\epsilon}^{S}} K_{\epsilon}^{S}\left|\nabla T_{\epsilon}(x)-\nabla T_{0}(x)-\nabla_{y^{\prime}} T_{1}\left(x, \frac{x^{\prime}}{\epsilon}\right)\right|^{2} d x+\epsilon \int_{\Omega_{\epsilon}^{F}} K_{\epsilon}^{F}\left|\nabla T_{\epsilon}(x)-\nabla T_{0}(x)-\nabla_{y^{\prime}} T_{1}\left(x, \frac{x^{\prime}}{\epsilon}\right)\right|^{2} d x \\
& +\frac{\sigma}{\epsilon} \int_{\Gamma_{\epsilon}} G_{\epsilon}\left(T_{\epsilon}(x)-T_{0}(x)-\epsilon T_{1}\left(x, \frac{x^{\prime}}{\epsilon}\right)\right)\left(T_{\epsilon}(x)-T_{0}(x)-\epsilon T_{1}\left(x, \frac{x^{\prime}}{\epsilon}\right)\right) d x=\int_{\Omega_{\epsilon}^{S}} f_{\epsilon}(x) T_{\epsilon}(x) d x \\
& +\int_{\Omega_{\epsilon}^{S}} K_{\epsilon}^{S}\left|\nabla T_{0}(x)+\nabla_{y^{\prime}} T_{1}\left(x, \frac{x^{\prime}}{\epsilon}\right)\right|^{2} d x+\epsilon \int_{\Omega_{\epsilon}^{F}} K_{\epsilon}^{F}\left|\nabla T_{0}(x)+\nabla_{y^{\prime}} T_{1}\left(x, \frac{x^{\prime}}{\epsilon}\right)\right|^{2} d x \\
& +\frac{\sigma}{\epsilon} \int_{\Gamma_{\epsilon}} G_{\epsilon}\left(T_{0}(x)+\epsilon T_{1}\left(x, \frac{x^{\prime}}{\epsilon}\right)\right)\left(T_{0}(x)+\epsilon T_{1}\left(x, \frac{x^{\prime}}{\epsilon}\right)\right) d x-2 \frac{\sigma}{\epsilon} \int_{\Gamma_{\epsilon}} G_{\epsilon}\left(T_{0}(x)+\epsilon T_{1}\left(x, \frac{x^{\prime}}{\epsilon}\right)\right) T_{\epsilon}(x) d x \\
& -2 \int_{\Omega_{\epsilon}^{S}} K_{\epsilon}^{S} \nabla T_{\epsilon}(x) \cdot\left(\nabla T_{0}(x)+\nabla_{y^{\prime}} T_{1}\left(x^{\prime}, \frac{x}{\epsilon}\right)\right) d x-2 \epsilon \int_{\Omega_{\epsilon}^{F}} K_{\epsilon}^{F} \nabla T_{\epsilon}(x) \cdot\left(\nabla T_{0}(x)+\nabla_{y^{\prime}} T_{1}\left(x, \frac{x^{\prime}}{\epsilon}\right)\right) d x
\end{aligned}
$$

Using the coercivity condition of $K_{\epsilon}^{S, F}$ on the left hand side, as well as the positivity of the operator $G_{\epsilon}$, and passing to the two scale limit in the right hand side of (81) we obtain an upper bound for

$$
\alpha \lim _{\epsilon \rightarrow 0}\left\|\left(\nabla T_{\epsilon}-\nabla T_{0}-\nabla_{y^{\prime}} T_{1}\right) \chi_{\epsilon}^{S}\right\|_{L^{2}(\Omega)}^{2}+\alpha \lim _{\epsilon \rightarrow 0} \epsilon\left\|\left(\nabla T_{\epsilon}-\nabla T_{0}-\nabla_{y^{\prime}} T_{1}\right) \chi_{\epsilon}^{F}\right\|_{L^{2}(\Omega)}^{2}
$$


Then, a combination of Lemmas 4.1 and 4.3 yields a bound for

$$
\lim _{\epsilon \rightarrow 0}\left\|T_{\epsilon}-T_{0}-\epsilon T_{1}\right\|_{L^{2}(\Omega)}^{2}
$$

We now have to prove that these upper bounds are all zero, i.e., that the two-scale limit of the right hand side of (81) vanishes.

Indeed, by virtue of (42) and (79) we have

$$
\begin{aligned}
\lim _{\epsilon \rightarrow 0} \frac{\sigma}{\epsilon} \int_{\Gamma_{\epsilon}} G_{\epsilon}\left(T_{0}(x)+\epsilon T_{1}\left(x, \frac{x^{\prime}}{\epsilon}\right)\right)\left(T_{0}(x)+\epsilon T_{1}\left(x, \frac{x^{\prime}}{\epsilon}\right)\right) d x \\
=\frac{\sigma}{|\Lambda|}\left(\frac{1}{2} \int_{\Omega} \frac{\partial T_{0}}{\partial x_{3}}(x) \frac{\partial T_{0}}{\partial x_{3}}(x) d x \int_{\gamma} \int_{\gamma} F^{2 D}\left(z^{\prime}, y^{\prime}\right)\left|z^{\prime}-y^{\prime}\right|^{2} d y^{\prime} d z^{\prime}\right. \\
\quad+\int_{\Omega} \int_{\gamma}\left(\nabla_{x^{\prime}} T_{0}(x) \cdot y+T_{1}\left(x, y^{\prime}\right)\right)\left(I d-\zeta^{2 D}\right)\left(T_{1}\left(x, y^{\prime}\right)+\nabla_{x^{\prime}} T_{0}(x) \cdot y\right) d y^{\prime} d x \\
=\int_{\Omega} K^{*}(x)\left|\nabla_{x} T_{0}(x)\right|^{2} d x-\frac{1}{|\Lambda|} \int_{\Omega} \int_{\Lambda^{S}} K^{S}\left(x, y^{\prime}\right)\left|\nabla_{x} T_{0}(x)+\nabla_{y^{\prime}} T_{1}\left(x, y^{\prime}\right)\right|^{2} d y^{\prime} d x
\end{aligned}
$$

and

$$
\begin{array}{r}
\lim _{\epsilon \rightarrow 0} \frac{\sigma}{\epsilon} \int_{\Gamma_{\epsilon}} G_{\epsilon}\left(T_{0}(x)+\epsilon T_{1}\left(x, \frac{x^{\prime}}{\epsilon}\right)\right) T_{\epsilon}(x) d x=\int_{\Omega} K^{*}(x)\left|\nabla_{x} T_{0}(x)\right|^{2} d x \\
-\frac{1}{|\Lambda|} \int_{\Omega} \int_{\Lambda^{S}} K^{S}\left(x, y^{\prime}\right)\left|\nabla_{x} T_{0}(x)+\nabla_{y^{\prime}} T_{1}\left(x, y^{\prime}\right)\right|^{2} d y^{\prime} d x
\end{array}
$$

Passing to the two scales limit in the right hand side of (81) yields

$$
-\int_{\Omega} K^{*}(x) \nabla_{x} T_{0}(x) \cdot \nabla_{x} T_{0}(x) d x+\theta \int_{\Omega} f(x) T_{0}(x) d x
$$

which is equal to zero thanks to the variational formulation of the homogenized problem (25). Hence the result.

\section{Non-linear case}

As already discussed in Remark 2.2, the true physical problem involves a non-linear radiation operator, defined by formula (9) instead of (5). The study of the linear case was a simplifying assumption in order to rigorously prove the convergence of the homogenization process. However, the formal method of two-scale asymptotic expansion is perfectly valid in the non-linear case too (see [3] and [20] if necessary). In this section we give, without proofs, the homogenization result in the non-linear case.

When the radiation operator, defined by formula is given by (9) instead of (5), the non-linear equivalent of Proposition 3.1 is the following.

Proposition 5.1. Under assumption (23) the leading term $T_{0}$ is the solution of the homogenized problem

$$
\left\{\begin{aligned}
-\operatorname{div}\left(K^{*}\left(x, T_{0}^{3}\right) \nabla T_{0}(x)\right)+V^{*}(x) \cdot \nabla T_{0}(x) & =\theta f(x) & & \text { in } \Omega \\
T_{0}(x) & =0 & & \text { on } \partial \Omega
\end{aligned}\right.
$$


with the porosity factor $\theta=\left|\Lambda^{S}\right| /|\Lambda|$, the homogenized conductivity given by its entries, for $j, k=$ $1,2,3$,

$$
\begin{array}{r}
K_{j, k}^{*}\left(x, T_{0}^{3}\right)=\frac{1}{|\Lambda|}\left[\int_{\Lambda^{S}} K^{S}\left(x, y^{\prime}\right)\left(e_{j}+\nabla_{y} \omega_{j}\left(y^{\prime}\right)\right) \cdot\left(e_{k}+\nabla_{y} \omega_{k}\left(y^{\prime}\right)\right) d y^{\prime}\right. \\
\quad+4 \sigma T_{0}^{3}(x) \int_{\gamma} G\left(\omega_{k}\left(y^{\prime}\right)+y_{k}\right)\left(\omega_{j}\left(y^{\prime}\right)+y_{j}\right) \\
\left.+2 \sigma T_{0}^{3}(x) \int_{\gamma} \int_{\gamma} F^{2 D}\left(s^{\prime}, y^{\prime}\right)\left|s^{\prime}-y^{\prime}\right|^{2} d y^{\prime} d s^{\prime} \delta_{j 3} \delta_{k 3}\right]
\end{array}
$$

and an homogenized velocity given by

$$
V_{k}^{*}=\frac{1}{|\Lambda|} \int_{\Lambda^{F}} V\left(x, y^{\prime}\right) \cdot e_{k} d y^{\prime}
$$

where $\left(\omega_{j}\left(x, T_{0}^{3}(x), y^{\prime}\right)\right)_{1 \leq j \leq 3}$ are the solutions of the cell problems

$$
\left\{\begin{aligned}
-\operatorname{div}_{y} K^{S}\left(x, y^{\prime}\right)\left(e_{j}+\nabla_{y} \omega_{j}^{S}\left(y^{\prime}\right)\right)=0 & \text { in } \Lambda^{S} \\
-\operatorname{div}_{y} K^{F}\left(x, y^{\prime}\right)\left(e_{j}+\nabla_{y} \omega_{j}^{F}\left(y^{\prime}\right)\right)+V\left(x, y^{\prime}\right) \cdot\left(e_{j}+\nabla_{y} \omega_{j}^{F}\left(y^{\prime}\right)\right)=0 & \text { in } \Lambda^{F} \\
-K^{S}\left(y^{\prime}, x_{3}\right)\left(e_{j}+\nabla_{y} \omega_{j}^{S}\left(y^{\prime}\right)\right) \cdot n=4 \sigma T_{0}^{3}(x) G\left(\omega_{j}^{S}\left(y^{\prime}\right)+y_{j}\right) & \text { on } \gamma \\
\omega_{j}^{F}\left(y^{\prime}\right)=\omega_{j}^{S}\left(y^{\prime}\right) & \text { on } \gamma \\
y^{\prime} \mapsto \omega_{j}\left(y^{\prime}\right) \text { is } \Lambda \text {-periodic, } &
\end{aligned}\right.
$$

and $T_{1}$ is given by

$$
T_{1}\left(x, y^{\prime}\right)=\sum_{j=1}^{3} \omega_{j}\left(x, T_{0}^{3}(x), y^{\prime}\right) \frac{\partial T_{0}}{\partial x_{j}}(x) .
$$

The homogenized problem (82) is a non-linear convection-diffusion model where the non-linearity appears only in the conductivity tensor $K^{*}$ which depends on the third power of the temperature. As usual in homogenization, the cell problems are linearized, depending on the value of the macroscopic temperature at each macroscopic point $x$. The linearization of the Stefan-Boltzmann law (giving the irradiance as the fourth power of the temperature) yields the coefficient $4 \sigma T_{0}^{3}$ in the radiation operator of the cell problem (83). Therefore, the cell solutions, as well as the homogenized tensor $K^{*}$, depend on the third power of the temperature $T_{0}^{3}$.

\section{Numerical results}

In this section we describe some numerical experiments to study the asymptotic behaviour of the heat transfer model (8) in the non-linear case, i.e., when the radiation operator is defined as in Remark 2.2. Our goal is to show the efficiency of our proposed homogenization procedure, to validate it by comparing the reconstructed solution of the homogenized model with the numerical solution of the exact model (8) for smaller and smaller values of $\epsilon$ and to exhibit a numerical rate of convergence in terms of $\epsilon$. While the computations in [3] were restricted to the 2D setting, here we perform 3D numerical simulations of (8). All computations have been done with the finite element code CAST3M [11] developed at the French Atomic and Alternative Energy Commission (CEA).

\subsection{Changing variables for the numerical simulation}

Usually, in homogenization theory, we solve a problem in a fixed domain $\Omega$ with cells of size $\epsilon$, which tends to 0 (see Figure 3). However, in practice for our nuclear reactor problem, the sizes of the 
gas cylinders and cell assemblies are fixed by manufacturing constraints. Therefore, following [3], we proceed differently: we fix the size of the periodical cell (independent of $\epsilon$ ) and we increase the total number of cells, i.e., the size of the global domain which is of order $\epsilon^{-1}$. In other words, instead of using the macroscopic space variable $x \in \Omega$, we use the microscopic space variable $y=x / \epsilon$. In this new frame of reference, all periodicity cells are of unit size and the computational domain is $\epsilon^{-1} \Omega$ which is increasing as $\epsilon$ goes to 0 (see Figure 4).

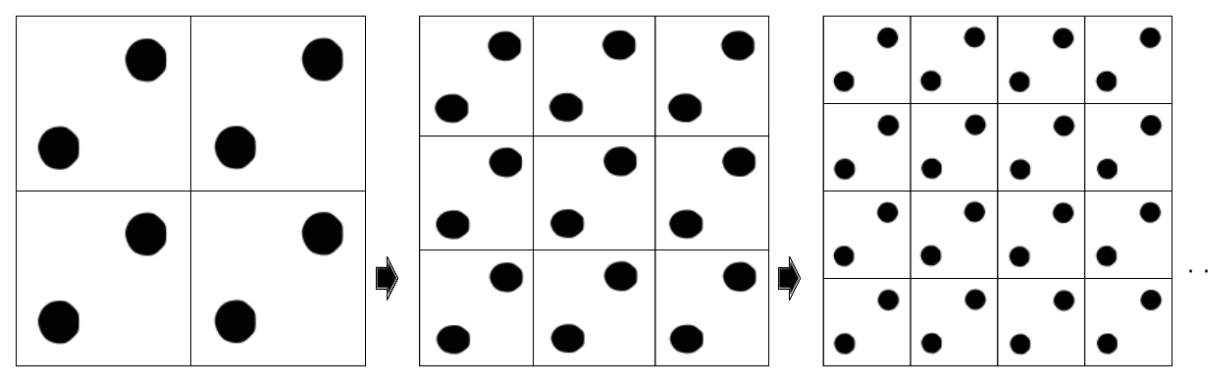

Figure 3: Standard homogenization in a fixed domain $\Omega$

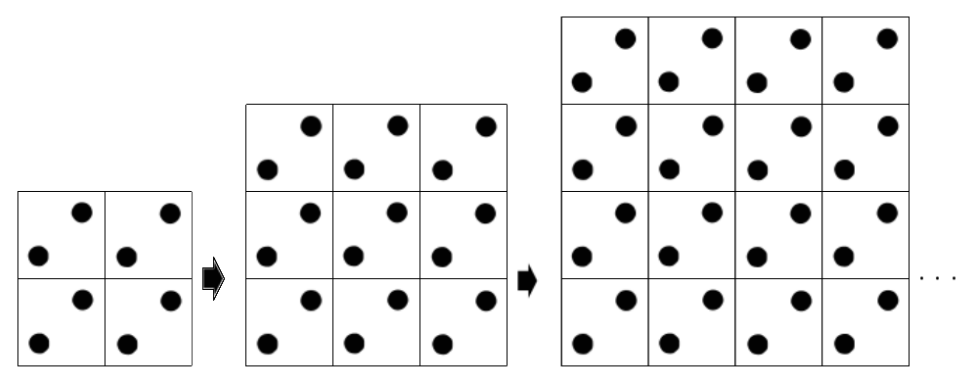

Figure 4: Rescaled process of homogenization with constant periodicity cell and increasing domain

$$
\widehat{\Omega}=\epsilon^{-1} \Omega
$$

If the fixed domain is denoted by $\Omega=\prod_{j=1}^{3}\left(0, L_{j}\right)$, our rescaled computational domain is $\widehat{\Omega}=$ $\epsilon^{-1} \Omega=\prod_{j=1}^{3}\left(0, L_{j} / \epsilon\right)$, where there exist integers $N_{j}$ such that $L_{j} / \epsilon=N_{j} \ell_{j}$, for $j=1,2$ (so that only entire cells belong to $\widehat{\Omega})$. For any function $u(x)$ defined on $\Omega$, we introduce the rescaled function $\widehat{u}(y)$, defined on $\widehat{\Omega}$ by

$$
\widehat{u}(y)=u(\epsilon y)=u(x),
$$

which satisfies $\nabla_{y} \widehat{u}(y)=\epsilon\left(\nabla_{x} u\right)(\epsilon y)=\epsilon \nabla_{x} u(x)$. All quantities defined in $\widehat{\Omega}$ are denoted with a hat ${ }^{\wedge}$ and, for simplicity, we drop the dependence on $\epsilon$. For example, we define the conductivity tensor $\widehat{K}$ as

$$
\widehat{K}(y)= \begin{cases}\widehat{K^{S}}(y)=K^{S}\left(\epsilon y, y^{\prime}\right) & \text { in } \widehat{\Omega}^{S}, \\ \widehat{K^{F}}(y)=\epsilon K^{F}\left(\epsilon y, y^{\prime}\right) & \text { in } \widehat{\Omega}^{F},\end{cases}
$$

and the fluid velocity

$$
\widehat{V}(y)=\epsilon V\left(\epsilon y, y^{\prime}\right) \quad \text { in } \widehat{\Omega}^{F} .
$$

We also define $\widehat{\Omega}^{S}, \widehat{\Omega}^{F}, \widehat{\Gamma}$ and $\partial \widehat{\Omega}$ by the same change of variables relating $\Omega$ and $\widehat{\Omega}$. In this new frame 
of reference, problem (8) becomes

$$
\left\{\begin{array}{rlrl}
-\operatorname{div}\left(\widehat{K^{S}} \nabla \widehat{T}_{\epsilon}\right) & =\epsilon^{2} \widehat{f} & \text { in } \widehat{\Omega}^{S} \\
-\operatorname{div}\left(\widehat{K^{F}} \nabla \widehat{T}_{\epsilon}\right)+\widehat{V} \cdot \nabla \widehat{T}_{\epsilon} & =0 & \text { in } \widehat{\Omega}^{F} \\
-\widehat{K^{S}} \nabla \widehat{T}_{\epsilon} \cdot n & =-\widehat{K^{F}} \nabla \widehat{T}_{\epsilon} \cdot n+\sigma G\left(\widehat{T}_{\epsilon}^{4}\right) & & \text { on } \widehat{\Gamma} \\
\widehat{T}_{\epsilon} & =0 & & \text { on } \partial \widehat{\Omega} \\
\widehat{T}_{\epsilon} & \text { is continuous through } \widehat{\Gamma} . &
\end{array}\right.
$$

The homogenized problem (82) becomes

$$
\left\{\begin{aligned}
-\operatorname{div}\left(\widehat{K}^{*}\left(\widehat{T}_{0}^{3}\right) \nabla \widehat{T_{0}}\right)+\epsilon \widehat{V}^{*} \cdot \nabla \widehat{T_{0}} & =\epsilon^{2} \theta \widehat{f} & \text { in } \widehat{\Omega} \\
\widehat{T_{0}} & =0 & \text { on } \partial \widehat{\Omega} .
\end{aligned}\right.
$$

Furthermore, we also define

$$
\widehat{T_{1}}(y)=\epsilon T_{1}\left(\epsilon y, y^{\prime}\right)=\epsilon T_{1}\left(x, \frac{x^{\prime}}{\epsilon}\right)=\sum_{i=1}^{3} \frac{\partial \widehat{T_{0}}}{\partial y_{i}}(y) \omega_{i}\left(y^{\prime}\right)
$$

where $\widehat{T_{1}}$ is purposely scaled as $\epsilon$ so that the $\epsilon$-factor disappears in the last equality of (90). Finally, the homogenization approximation $T_{\epsilon}(x) \simeq T_{0}(x)+\epsilon T_{1}(x, x / \epsilon)$ becomes

$$
\widehat{T_{\epsilon}}(y) \simeq \widehat{T_{0}}(y)+\widehat{T_{1}}(y) .
$$

Since a factor $\epsilon^{\frac{d}{2}}$ appears when changing variables $y=x / \epsilon$ in the $L^{2}$-norms, we compute relative errors between the exact and reconstructed solutions in the sequel. The relative errors are invariant by our change of variables

$$
\frac{\left\|T_{\epsilon}(x)-\left(T_{0}(x)+\epsilon T_{1}\left(x, \frac{x}{\epsilon}\right)\right)\right\|_{L^{2}(\Omega)}}{\left\|T_{\epsilon}(x)\right\|_{L^{2}(\Omega)}}=\frac{\left\|\widehat{T}_{\epsilon}(y)-\left(\widehat{T_{0}}(y)+\widehat{T_{1}}(y)\right)\right\|_{L^{2}(\widehat{\Omega})}}{\left\|\widehat{T}_{\epsilon}(y)\right\|_{L^{2}(\widehat{\Omega})}}
$$

and

$$
\frac{\left\|\nabla T_{\epsilon}(x)-\nabla\left(T_{0}(x)+\epsilon T_{1}\left(x, \frac{x}{\epsilon}\right)\right)\right\|_{L^{2}(\Omega)}}{\left\|\nabla T_{\epsilon}(x)\right\|_{L^{2}(\Omega)}}=\frac{\left\|\nabla \widehat{T}_{\epsilon}(y)-\nabla\left(\widehat{T_{0}}(y)+\widehat{T_{1}}(y)\right)\right\|_{L^{2}(\widehat{\Omega})}}{\left\|\nabla \widehat{T}_{\epsilon}(y)\right\|_{L^{2}(\widehat{\Omega})}}
$$

\subsection{Algorithm and computational parameters}

We first give our methodology for the numerical simulations of the homogenization process.

1. Solve the 3 cell problems (83) for a range of values of $\widehat{T}_{0}$ (see Figure 5).

2. Compute the homogenized conductivity (as a function of temperature) and the homogenized velocity.

3. Solve the homogenized problem (82) by a fixed point algorithm (see Figure 7).

4. Compute the corrector $\widehat{T}_{1}(y)=\sum_{i=1}^{3} \frac{\partial \widehat{T_{0}}}{\partial y_{i}}(y) \omega_{i}\left(y^{\prime}\right)$.

5. Reconstruct an approximate solution: $\widehat{T}_{0}(y)+\widehat{T}_{1}(y)$ (see Figure 7$)$. 
We now give our computational parameters for a reference computation corresponding to $\epsilon=\epsilon_{0}=$ $\frac{1}{4}$. The geometry corresponds to a cross-section of a typical fuel assembly for a gas-cooled nuclear reactor (see [20] for further reference). The domain is $\widehat{\Omega}=\epsilon^{-1} \Omega=\prod_{j=1}^{3}\left(0, L_{j} / \epsilon\right)$, with $L_{3}=0.025 \mathrm{~m}$ and, for $j=1,2, L_{j} / \epsilon=N_{j} \ell_{j}$ where $N_{1}=3, N_{2}=4$ and $\ell_{1}=0.04 m, \ell_{2}=0.07 m$. Each periodicity cell contains 2 hollow cylinders (holes) (see Figure 1), the radius of which is equal to $0.0035 \mathrm{~m}$. The emissivity of the holes boundaries is equal to $e=1$. The thermal source $f$ is set to zero (we refer to [21] for other computations, including ones with $f \neq 0$ ). We enforce periodic boundary conditions in the $x_{1}$ direction and non-homogeneous Dirichlet boundary conditions in the other directions which are given by $\widehat{T}_{\epsilon}(y)=\epsilon\left(3200 y_{1}+400 y_{2}\right)+800$ on the boundaries corresponding to $y_{2}=0, y_{2}=L_{2} / \epsilon, y_{3}=0$ and $y_{3}=L_{3} / \epsilon$. This boundary condition depends on $\epsilon$, as a function of $y$, in such way that, as a function of $x=\epsilon y$, it is independent of $\epsilon$.

The physical values of the isotropic conductivity are $30 \mathrm{Wm}^{-1} \mathrm{~K}^{-1}$ in the solid part and $0.3 \mathrm{Wm}^{-1} \mathrm{~K}^{-1}$ in the fluid part. Since it is much smaller in the fluid than in the solid, we decided to scale it by $\epsilon$, see (3) and (86). In other words, the conductivity tensor defined in (86) takes the values

$$
\widehat{K}= \begin{cases}30 W m^{-1} K^{-1} & \text { in } \widehat{\Omega}^{S} \\ \frac{\epsilon}{\epsilon_{0}} 0.3 \mathrm{Wm}^{-1} K^{-1} & \text { in } \widehat{\Omega}^{F}\end{cases}
$$

On a similar token, the physical value of the fluid velocity (assumed to be constant and parallel to the cylinders axis) is $80 \mathrm{~ms}^{-1}$. By the scaling of (87), the numerical value of the velocity is

$$
\widehat{V}=\left(\begin{array}{l}
0 \\
0 \\
\frac{\epsilon}{\epsilon_{0}} 80
\end{array}\right) m s^{-1} .
$$

Remark that it is only for the reference computation $\epsilon_{0}=1 / 4$ that $\widehat{K}$ and $\widehat{V}$ are equal to their physical values. While the rescaled coefficients $\widehat{K^{F}}(y)$ and $\widehat{V}(y)$ are varying with $\epsilon$, the original coefficients $K^{F}(x)$ and $V(x)$ are independent of $\epsilon$. The fact that the numerical values of $\widehat{K}^{F}$ and $\widehat{V}$ are not the physical ones for $\epsilon \neq \epsilon_{0}=1 / 4$ is not a problem, since our convergence study (as $\epsilon$ goes to 0 ) is purely a numerical verification of our mathematical result.

As explained in Section 6.1 we shall check numerically the convergence of the homogenization process when $\epsilon$ goes to zero, or more precisely when the number of cells goes to infinity. We thus compare the solution $\widehat{T}_{\epsilon}$ of (8) (obtained by a costly numerical computation) with the homogenized reconstructed solution $\widehat{T}_{0}(y)+\widehat{T}_{1}(y)$ (which is much cheaper to compute). Furthermore, we shall obtain speed of convergences for the relative errors (92) and (93) plotted in Figures 11 and 12. To avoid an excessive computational burden, we have chosen periodic boundary condition in the $x_{1}$ direction which implies that it is not necessary to add cells in the $x_{1}$ direction. Therefore, $N_{1}=3$ is fixed and we simply add cells in the $x_{2}$ direction, increasing $N_{2}$ from 4 to 10 with a unit step. In other words, we define

$$
\epsilon=\frac{1}{N_{2}}
$$

Note that the vertical size of $\widehat{\Omega}$ is $L_{3} / \epsilon$, which is thus increasing as $\epsilon$ goes to zero.

All computations are performed with rectangular $\mathbb{Q}_{1}$ finite elements (4 nodes in $2 \mathrm{D}, 8$ nodes in 3D). A boundary integral method is used for the radiative term (which involves a dense matrix coupling all nodes on the surface enclosing a fluid part). The typical number of nodes for the $2 \mathrm{D}$ cell problem is 1027 (from which 72 are on the radiative boundary $\gamma$ ); it is 6336 for the 3D homogenized problem (which has no radiative term); it is 96480 for the original problem (8) with $\epsilon=\epsilon_{0}=\frac{1}{4}$ (from which 6912 are on the radiative boundary $\Gamma_{\epsilon}$ ).

\subsection{Simulation results}

In Figure 5 we plot the solutions of the cell problems (83) for an homogenized temperature $T_{0}=800 \mathrm{~K}$. Recall that, in the non linear case, the solutions of the cell problems depend on the macroscopic 
temperature. We recognize that $\omega_{3}^{S}$ is a constant in Figure 5 (right).

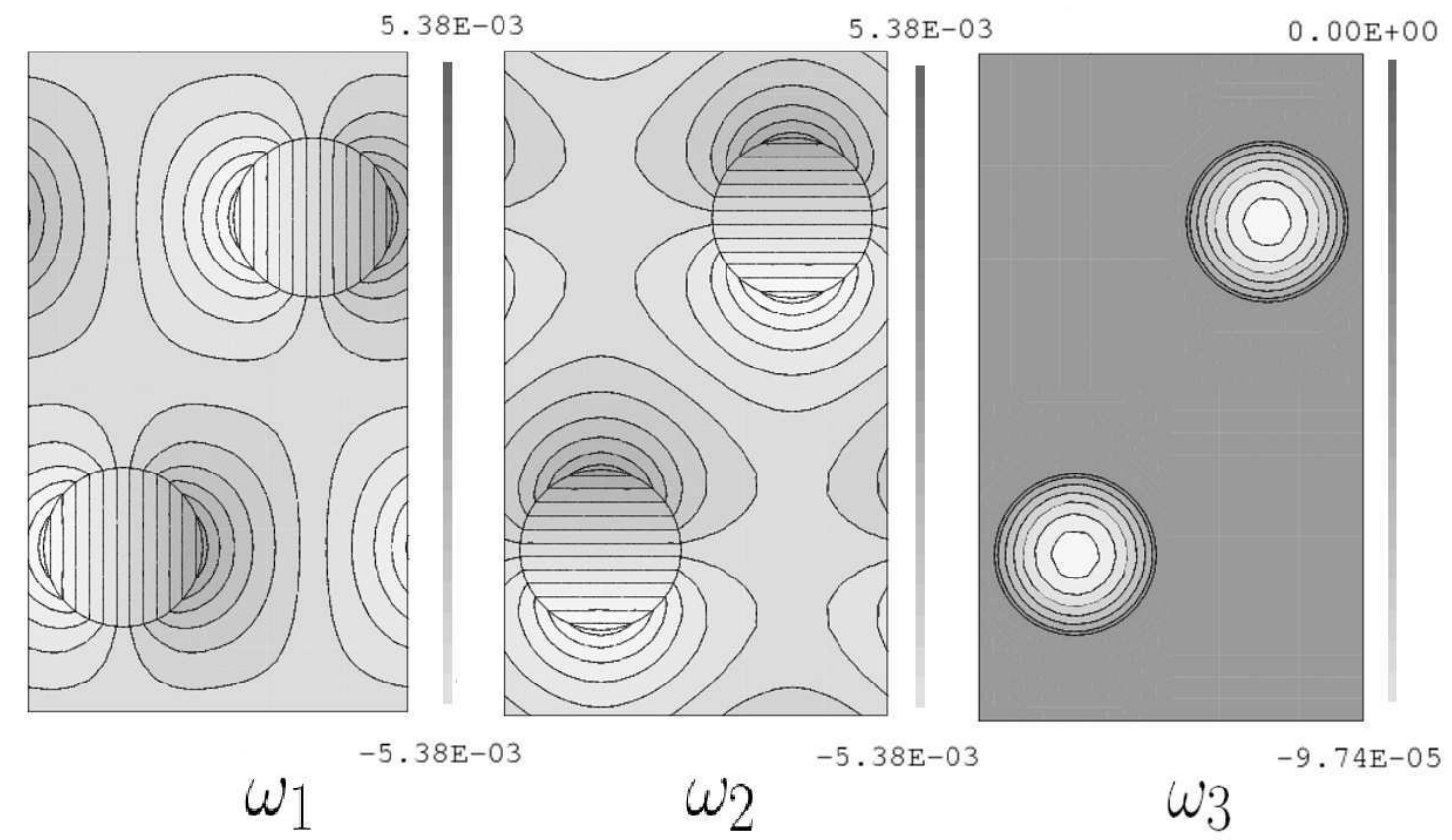

Figure 5: Solutions of the cell problems for $T_{0}=800 \mathrm{~K}$

The cell solutions allow us to evaluate the homogenized conductivity which turns out to numerically be a diagonal tensor (at least for temperatures $T_{0} \leq 1 E+05 K$ with a precision on 14 digits). However, for larger (extreme) temperatures, $\widehat{K}^{*}$ is not any longer a diagonal tensor [3]. The diagonal entries of $\widehat{K}^{*}$ are plotted on Figure 6 and two typical values are

$\widehat{K}^{*}\left(T_{0}=50 K\right)=\left(\begin{array}{lll}25.907 & 0 . & 0 . \\ 0 . & 25.914 & 0 . \\ 0 . & 0 . & 30.05\end{array}\right), \quad \widehat{K}^{*}\left(T_{0}=20000 K\right)=\left(\begin{array}{lll}49.801 & 0 . & 0 . \\ 0 . & 49.781 & 0 . \\ 0 . & 0 . & 3680.7\end{array}\right)$.

The homogenized velocity is a simple volume average, equal to

$$
\widehat{V}^{*}=\left(\begin{array}{l}
0 \\
0 \\
15.134
\end{array}\right) m s^{-1}
$$




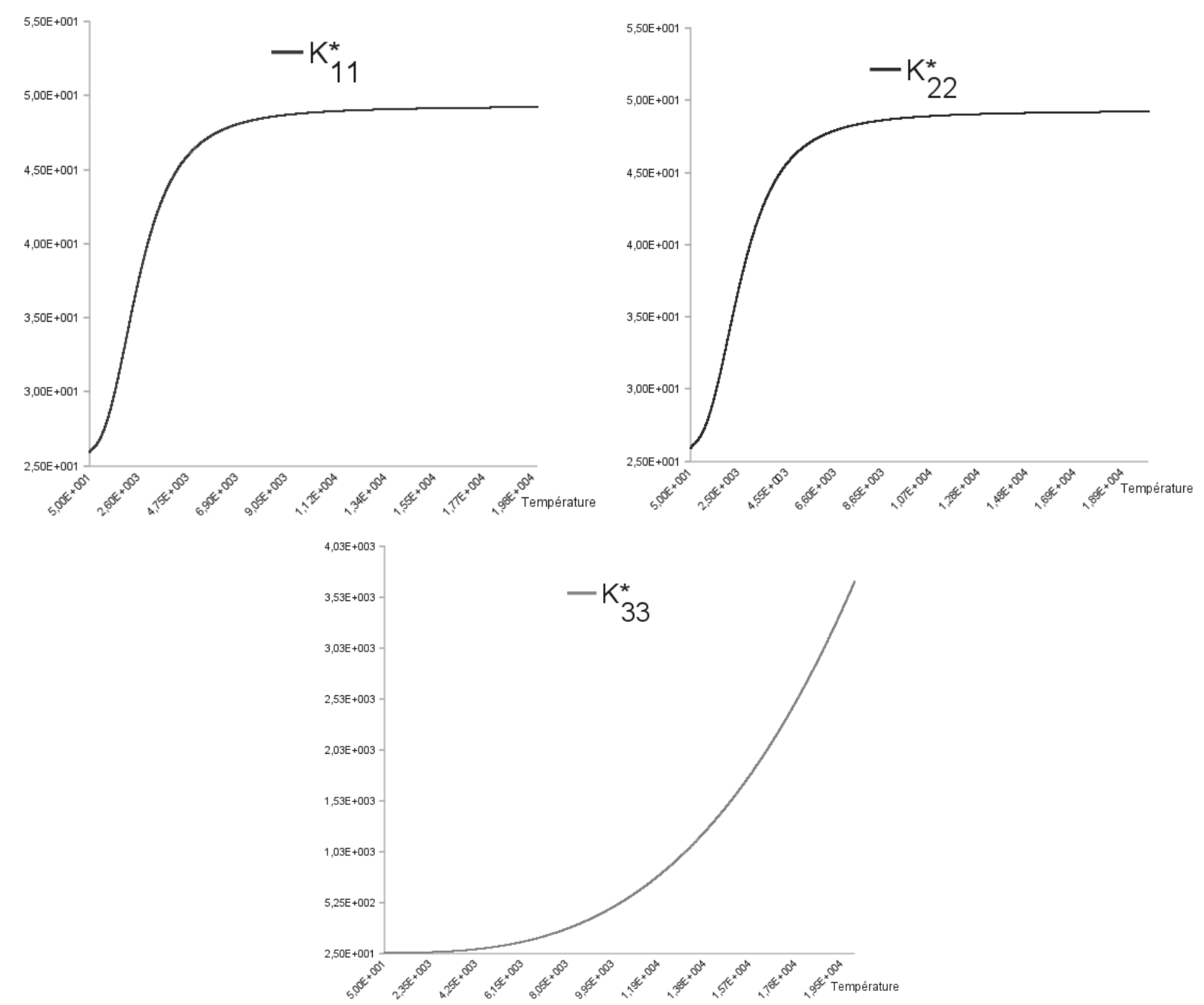

Figure 6: Homogenized conductivities as a function of the macroscopic temperature: $K_{11}^{*}$ (top left), $K_{22}^{*}$ (top right), $K_{33}^{*}$ (bottom).

By a fixed point algorithm (the homogenized conductivity $\widehat{K}^{*}$ is evaluated with the previous iterate for the temperature), we solve the homogenized problem (it requires of the order of 5 iterates). By a Newton method we solve also the direct model (8) (it requires of the order of 15 iterates). In Figure 7 we plot the direct, homogenized and reconstructed solutions computed for a value of $\epsilon=\epsilon_{0}=1 / 4$, as well as the error between the direct and reconstructed temperature. The error is clearly small and mostly concentrated on the domain boundaries. The moduli of the temperature gradients are displayed on Figure 8. Clearly the reconstructed solution $\widehat{T}_{0}+\widehat{T}_{1}$ is a much better approximation of the true solution $\widehat{T}_{\epsilon}$ than the mere homogenized solution $\widehat{T}_{0}$. The error on the temperature gradient is larger and again concentrated on the domain boundaries (this is consistent with the presence of boundary layers not taken into account in our asymptotic analysis). 

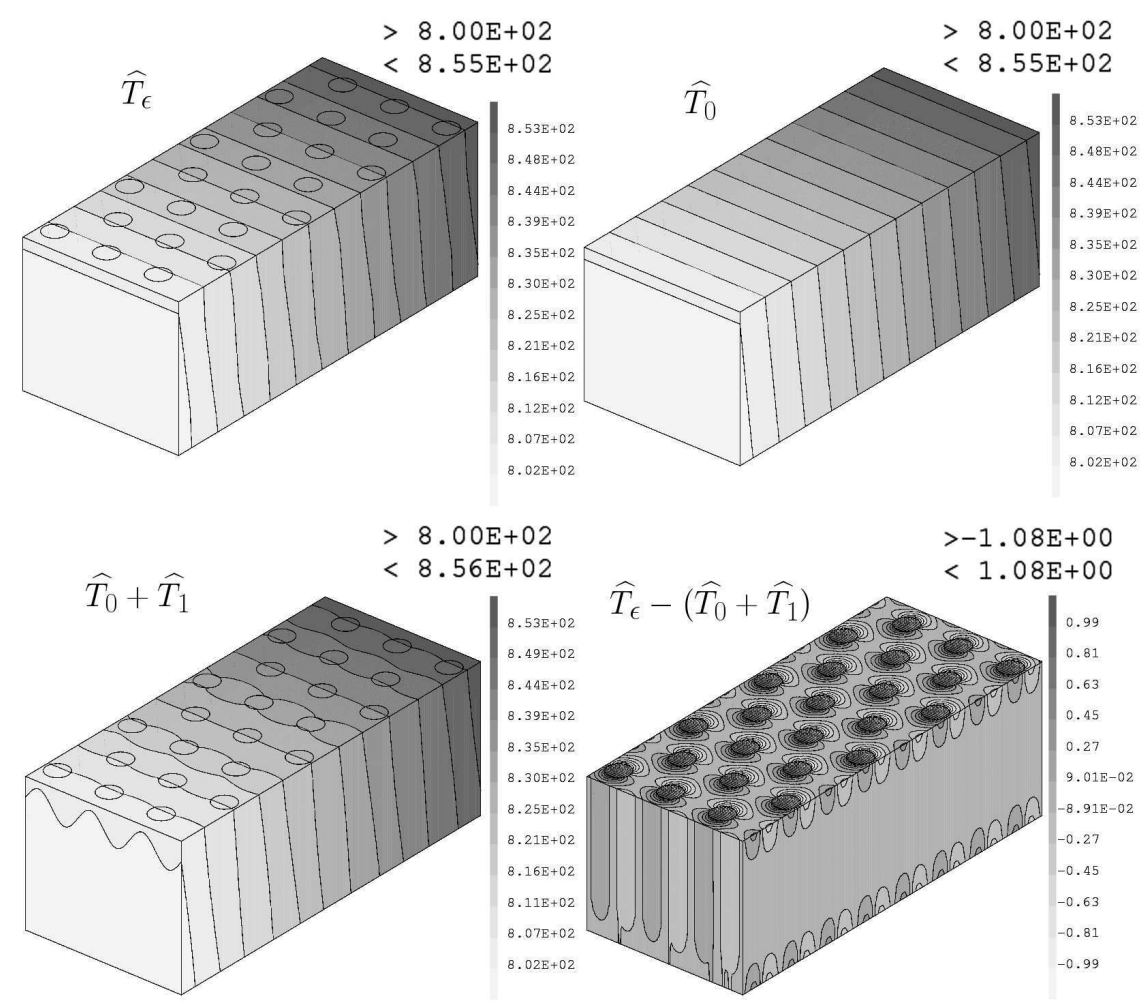

Figure 7: Solutions in $\widehat{\Omega}$
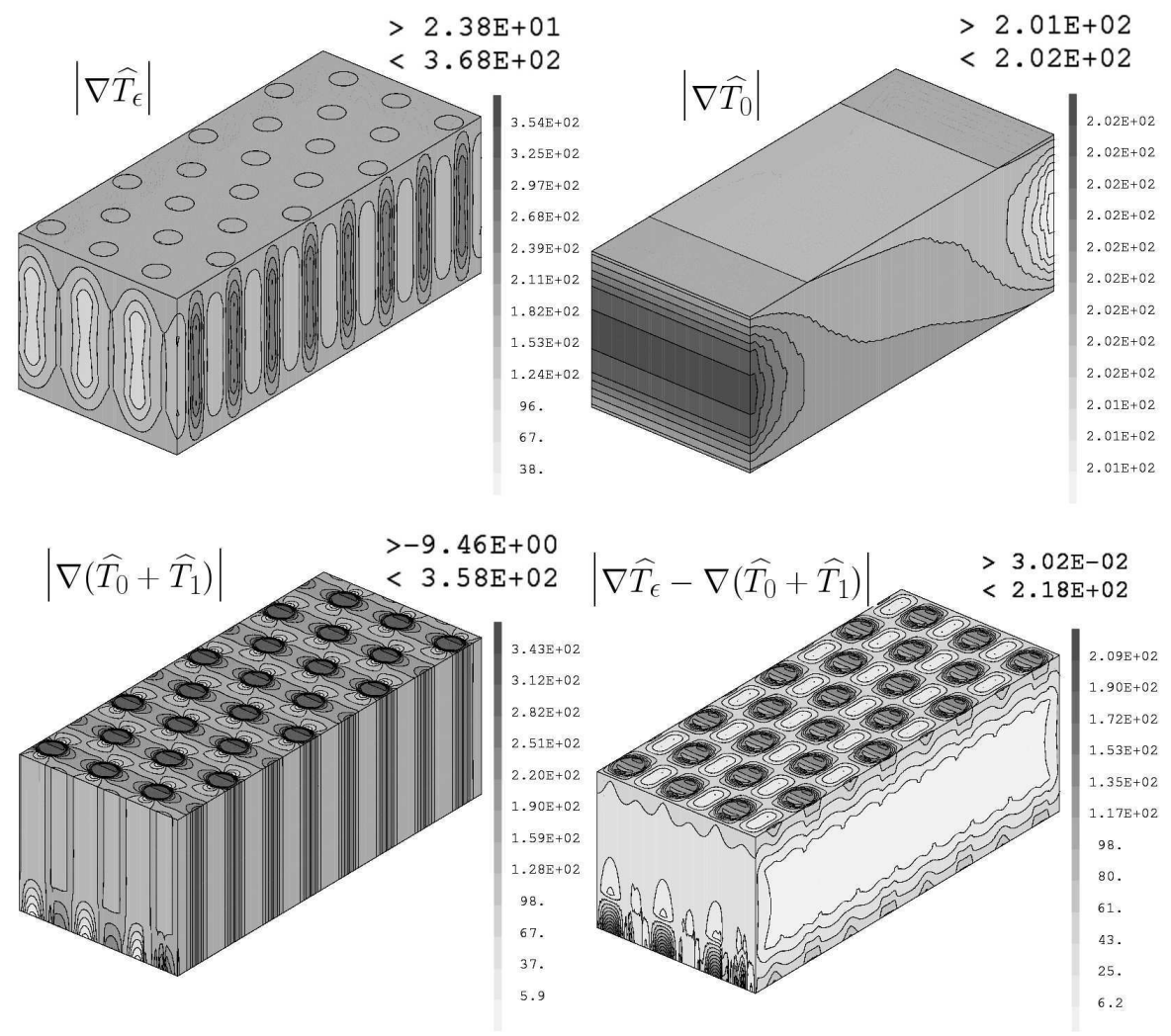

Figure 8: Modules of the solution gradients in $\widehat{\Omega}$ 
Notice on Figure 7 that the reconstructed temperature is slightly fluctuating on the boundary $y_{2}=0$ while the true solution is linear. This is due to the fact that the corrector $\widehat{T}_{1}$ does not satisfy a Dirichlet boundary condition. This well-known effect in homogenization can be corrected by introducing further terms, called boundary layers [8], [10]. We shall not dwell on this issue, all the more since other boundary layers are involved in our approximation. Indeed, the dimension reduction which applies to the radiative operator (which is truly 3D in the direct model (8) and only $2 \mathrm{D}$ in the cell problems) certainly generates boundary layers close to the top and bottom boundaries $y_{3}=0$ and $y_{3}=L_{3} / \epsilon$. Nevertheless, if we plot the solutions in a smaller domain $\widehat{\Delta}$ (which is obtained from $\widehat{\Omega}$ by removing one row of cells close to each boundary face normal to the $x_{2}$ direction and a layer of thickness $0.025 \mathrm{~m}$ at the top and bottom faces) we obtain a better agreement between $\widehat{T}_{0}+\widehat{T}_{1}$ and $\widehat{T}_{\epsilon}$ (see Figure 9$)$ and between $\nabla\left(\widehat{T}_{0}+\widehat{T}_{1}\right)$ and $\nabla \widehat{T}_{\epsilon}$ (see Figure 10).
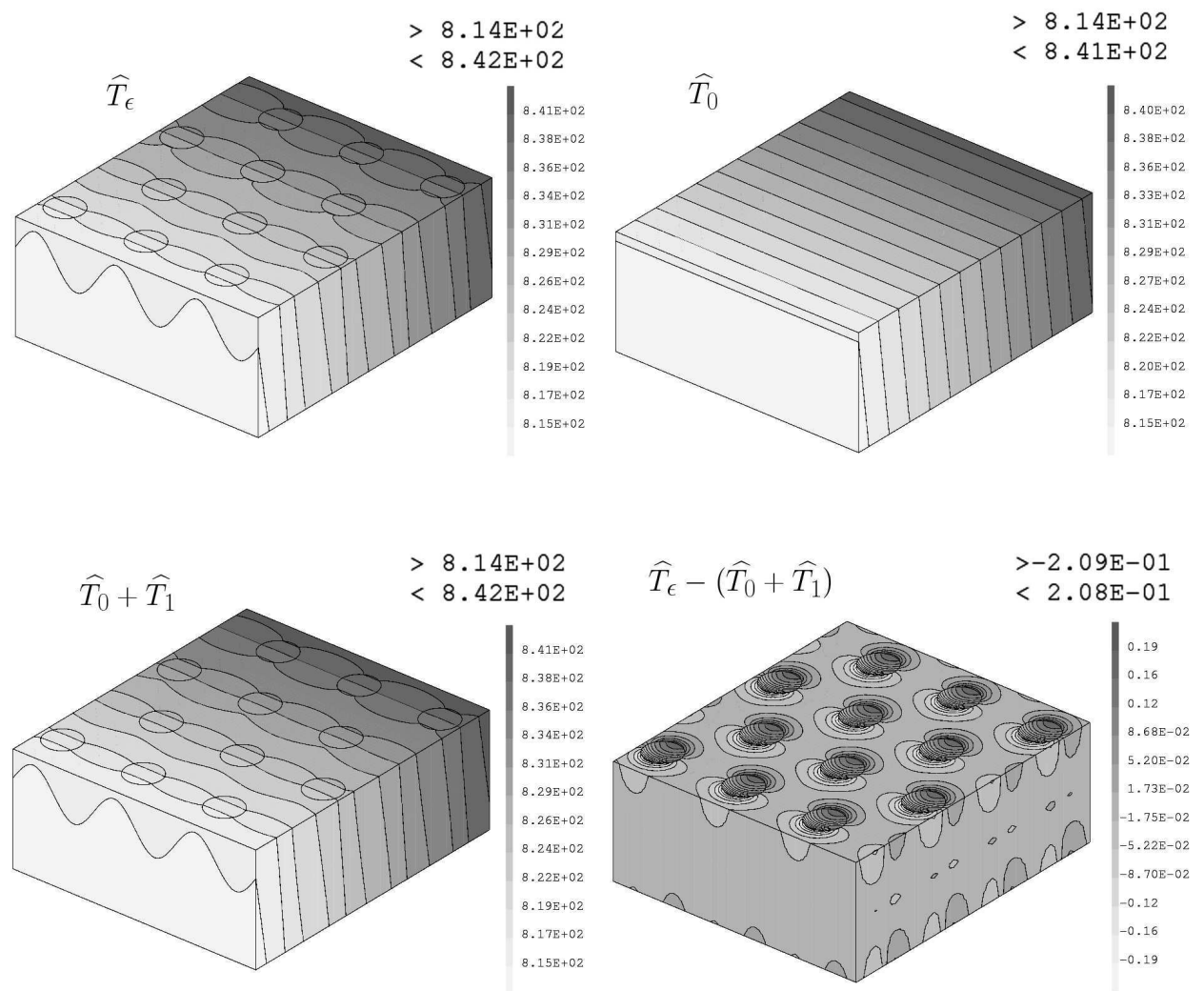

Figure 9: Solutions in the reduced domain $\widehat{\Delta}$

Now, to check the convergence of our homogenization process and to obtain a numerical speed of convergence, we display in Figures 11 and 12, as a function of $\epsilon$ on a log-log plot, the relative errors (92) and (93) related to temperature $E R R(T)$ and temperature gradient $E R R(\nabla T)$. We compare these errors with the slopes of $\epsilon$ and $\sqrt{\epsilon}$. This has to be compared with the classical error estimate for a pure diffusion problem (without radiative transfer) as given in [10]

$$
\left\{\begin{array}{l}
\operatorname{ERR}(T)_{\widehat{\Omega}}=\frac{\left\|\widehat{T}_{\epsilon}(y)-\left(\widehat{T}_{0}(y)+\widehat{T}_{1}(y)\right)\right\|_{L^{2}(\widehat{\Omega})}}{\left\|\widehat{T}_{\epsilon}(y)\right\|_{L^{2}(\widehat{\Omega})}} \leq C \epsilon, \\
\operatorname{ERR}(\nabla T)_{\widehat{\Omega}}=\frac{\left\|\nabla \widehat{T}_{\epsilon}(y)-\left(\nabla \widehat{T}_{0}(x)+\nabla \widehat{T}_{1}(y)\right)\right\|_{L^{2}(\widehat{\Omega})}}{\left\|\nabla \widehat{T}_{\epsilon}(y)\right\|_{L^{2}(\widehat{\Omega})}} \leq C \sqrt{\epsilon} .
\end{array}\right.
$$

Our errors $E R R(T)$ and $E R R(\nabla T)$ are in accordance with those theoretically predicted for a pure 

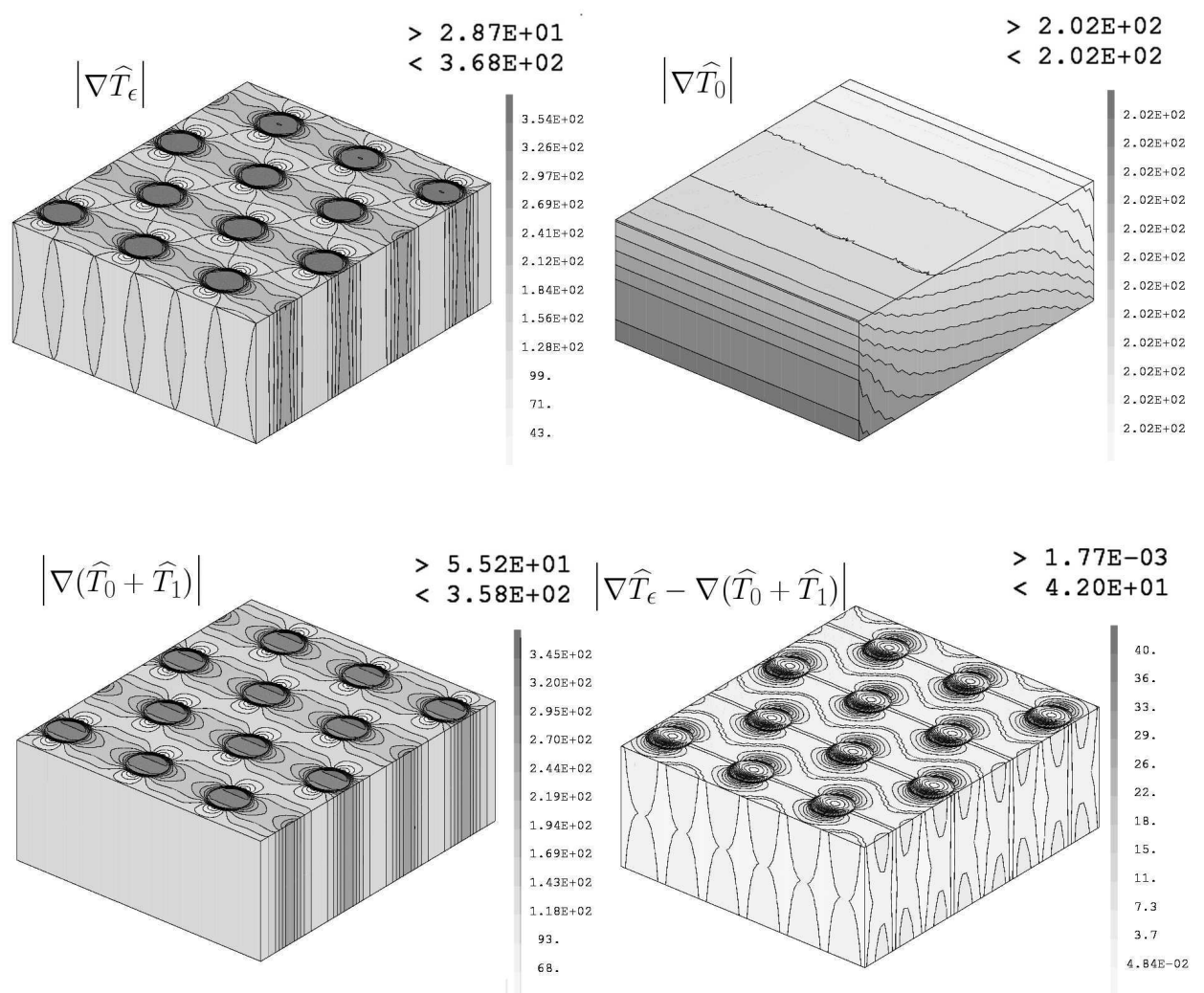

Figure 10: Modules of the solution gradients in the reduced domain $\widehat{\Delta}$

diffusion problem, namely they behave like $\epsilon$ and $\sqrt{\epsilon}$, respectively. In particular, it implies that the additional boundary layers caused by the dimension reduction effect (due to the radiative term) have an impact on the error compararable or smaller than that the homogenization boundary layers.

Even for moderate-size computations, like the ones in this section, the gain in memory and CPU time for our homogenization method is enormous compared to a direct simulation. This is a wellknown fact in the homogenization of diffusion problem but the gain is all the more extreme because of the radiative transfer involved in our model. Indeed, the direct model (8) involves a 3D radiative transfer operator which implies that full matrices connecting all nodes on the surface of one cylinder have to be stored and inverted (of course they are coupled through the diffusive rigidity matrix in the solid part). Typically, one Newton iteration in our reference computation takes about 80 min on a computer which has a memory of $37.2 G B$ and 12 processors with $C P U=2.67 G H z$. On the other hand, the cell problems (27) are merely 2D, so very cheap to solve (typically, one solution for a given temperature $T_{0}$ takes $18 . E-04 \mathrm{~min}$ with the same computer), and the homogenized problem (82) features no radiative term (one Newton iteration in our reference computation takes $12 . E-02 \mathrm{~min}$ with the same computer). Therefore, our algorithm of Subsection 6.2 is very competitive and is able to treat very large cases, like a full nuclear core computation. 


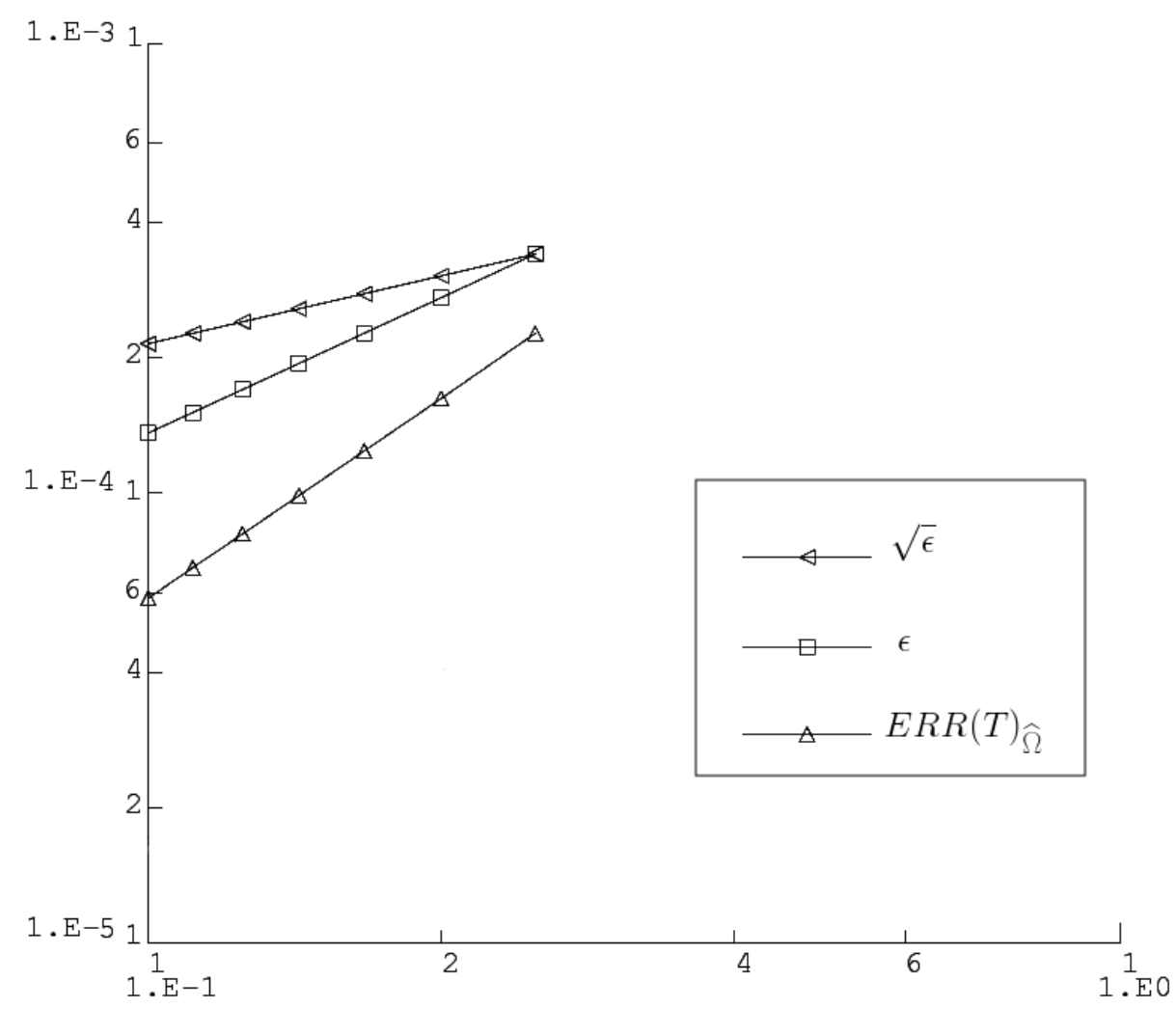

Figure 11: Relative error (92) for the temperature

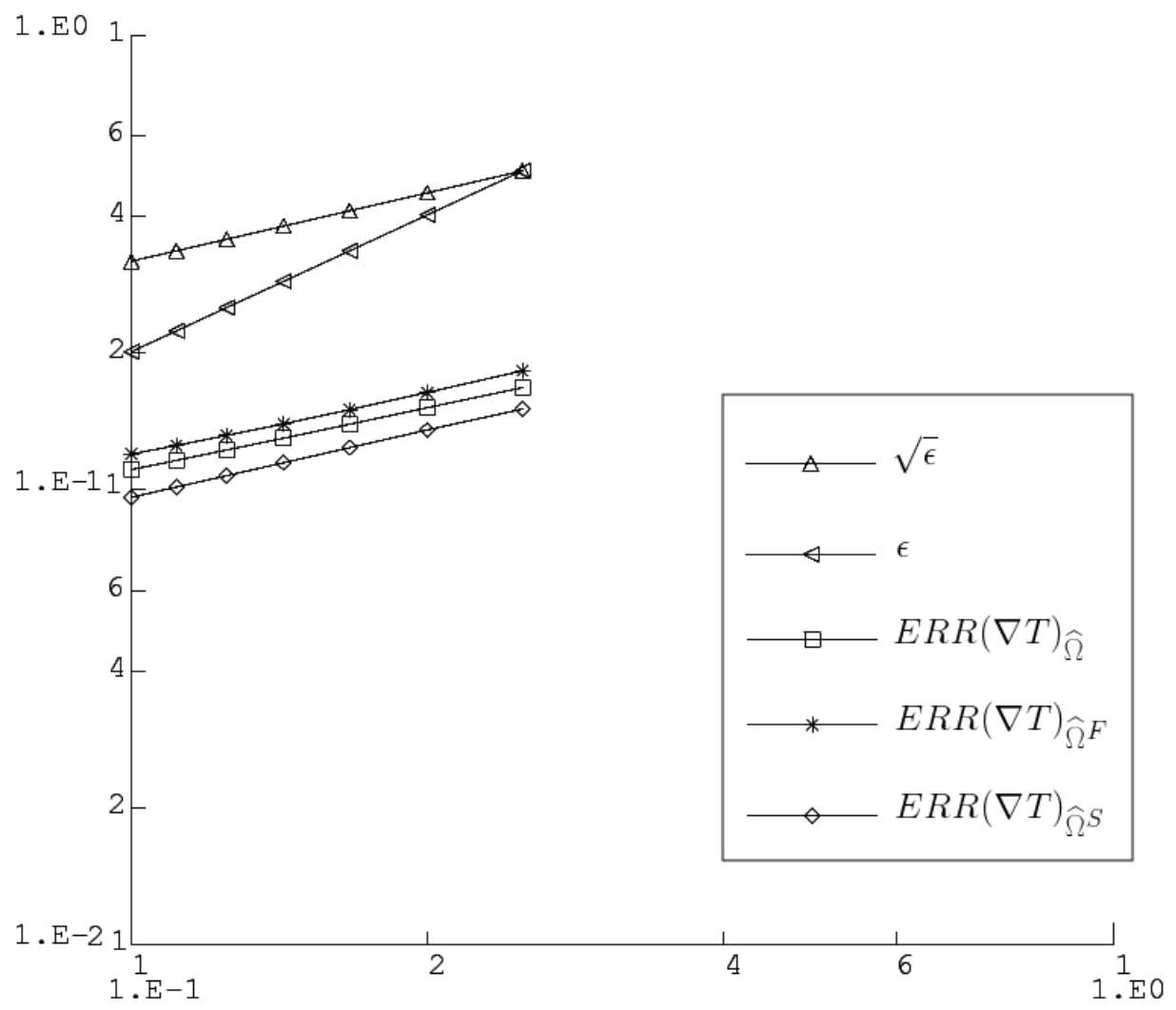

Figure 12: Relative error (93) for the temperature gradient 
Acknowledgement. The authors thank A. Stietel (CEA Saclay) for her useful collaboration.

\section{References}

[1] G. Allaire. Homogenization and two-scale convergence. SIAM J. Math. Anal., 23(6):1482-1518, 1992.

[2] G. Allaire, A. Damlamian, and U. Hornung. Two-scale convergence on periodic surfaces and applications. In Proceedings of the International Conference on Mathematical Modelling of Flow through Porous Media (May 1995), A. Bourgeat et al. eds. World Scientific Pub., pages 15-25, Singapore 1996.

[3] G. Allaire and K. El Ganaoui. Homogenization of a conductive and radiative heat transfer problem. Multiscale Model. Simul., 7(3):1148-1170, 2008.

[4] G. Allaire and Z. Habibi. Second order corrector in the homogenization of a conductive-radiative heat transfer problem. DCDS series B, 18(1):1-36, 2013.

[5] G. Allaire and F. Murat. Homogenization of the Neumann problem with nonisolated holes. Asymptotic Anal., 7(2):81-95, 1993. With an appendix written jointly with A. K. Nandakumar.

[6] A. A. Amosov. Nonstationary nonlinear nonlocal problem of radiative-conductive heat transfer in a system of opaque bodies with properties dependig on the radiation frequency. J. Math. Sci., New York 165, No. 1, 1-41 (2010).

[7] A. A. Amosov. Stationary nonlinear nonlocal problem of radiative-conductive heat transfer in a system of opaque bodies with properties depending on radiation frequency. J. Math. Sci., 164(3):309-344, 2010.

[8] N. Bakhvalov and G. Panasenko. Homogenisation: averaging processes in periodic media, volume 36 of Mathematics and its Applications (Soviet Series). Kluwer Academic Publishers Group, Dordrecht, 1989. Mathematical problems in the mechanics of composite materials, Translated from the Russian by D. Leĭtes.

[9] N. S. Bakhvalov. Averaging of the heat transfer process in periodic media in the presence of radiation. Differentsial' nye Uravneniya, 17(10):1765-1773, 1916, 1981.

[10] A. Bensoussan, J. L. Lions, and G. Papanicolaou. Asymptotic analysis for periodic structures, volume 5 of Studies in Mathematics and its Applications. North-Holland Publishing Co., Amsterdam, 1978.

[11] Cast3M. http://www-cast3m.cea.fr/cast3m/index.jsp.

[12] S. Chandrasekhar. Radiative transfer. Dover Publications Inc., New York, 1960.

[13] P. Charrier and B. Dubroca. Asymptotic transport models for heat and mass transfer in reactive porous media. Multiscale Model. Simul., 2(1):124-157 (electronic), 2003.

[14] P. G. Ciarlet. Plates and junctions in elastic multi-structures, volume 14 of Recherches en Mathématiques Appliquées [Research in Applied Mathematics]. Masson, Paris, 1990. An asymptotic analysis.

[15] D. Cioranescu and P. Donato. An introduction to homogenization, volume 17 of Oxford Lecture Series in Mathematics and its Applications. The Clarendon Press Oxford University Press, New York, 1999. 
[16] D. Cioranescu, P. Donato, and R. Zaki. Asymptotic behavior of elliptic problems in perforated domains with nonlinear boundary conditions. Asymptot. Anal., 53(4):209-235, 2007.

[17] G. Dal Maso. An introduction to $\Gamma$-convergence. Progress in Nonlinear Differential Equations and their Applications, 8. Birkhäuser Boston Inc., Boston, MA, 1993.

[18] P. Donato and L. Sgambati. Homogenization for some nonlinear problems in perforated domains. Revista de Matemàticas Aplicadas, 15(1):17-38, 1994.

[19] CEA e-den. Les réacteurs nucléaires à caloporteur gaz. CEA Saclay et Le Moniteur Editions. Monographie Den,2006. http://nucleaire.cea.fr/fr/publications/pdf/M0-fr.pdf.

[20] K. El Ganaoui. Homogénéisation de modèles de transferts thermiques et radiatifs : application au coeur des réacteurs à caloporteur gaz. PhD thesis, Ecole Polytechnique, 2006.

[21] Z. Habibi. Homogénéisation et convergence à deux échelles lors d'échanges thermiques stationnaires et transitoires dans un coeur de réacteur à caloporteur gaz. PhD thesis, Ecole Polytechnique, (in preparation).

[22] P.S. Heckbert. Simulating global illumination using Adaptive Meshing. PhD thesis, UC BerKeley, 1991.

[23] U. Hornung, editor. Homogenization and porous media, volume 6 of Interdisciplinary Applied Mathematics. Springer-Verlag, New York, 1997.

[24] J. R. Howell. A catalogue of radiation heat transfer factors. The university of Texas at Austin, Austin, Texas, 3 edition, 2010. http://www.engr.uky.edu/rtl/Catalog/.

[25] V. V. Jikov, S. M. Kozlov, and O. A. Oleinik. Homogenization of Differential Operators and Integral Functionals. Springer Verlag, 1994.

[26] J. L. Lions. Some methods in the mathematical analysis of systems and their control. Kexue Chubanshe (Science Press), Beijing, 1981.

[27] F. M. Modest. Radiative heat transfer. Academic Press, 2 edition, 2003.

[28] F. Murat and L. Tartar. H-convergence. In Topics in the mathematical modelling of composite materials, volume 31 of Progr. Nonlinear Differential Equations Appl., pages 21-43. Birkhäuser Boston, Boston, MA, 1997.

[29] M. Neuss-Radu. Some extensions of two-scale convergence. C. R. Acad. Sci. Paris Sér. I Math., 322(9):899-904, 1996.

[30] G. Nguetseng. A general convergence result for a functional related to the theory of homogenization. SIAM J. Math. Anal., 20(3):608-623, 1989.

[31] E. Sanchez-Palencia. Nonhomogeneous media and vibration theory, volume 127 of Lecture Notes in Physics. Springer-Verlag, Berlin, 1980.

[32] L. Tartar. The general theory of homogenization. A personalized introduction. Lecture Notes of the Unione Matematica Italiana. Springer Verlag, Berlin; UMI, Bologna, 7 edition, 2009.

[33] T. Tiihonen. Stefan-boltzmann radiation of nonlocal heat radiation problems. Math. Models Methods Appl. Sci., 8(6):1071-1089, 1998. 